\title{
DEVELOPMENT OF AN OPTICAL METHOD TO MEASURE THE THERMAL CONDUCTIVITY OF TRANSPARENT NANOFLUIDS
}

\author{
By \\ Winsle Anpalagan \\ BEng, Ryerson University, Canada, 2016 \\ A thesis \\ presented to Ryerson University \\ in partial fulfillment of the \\ requirement for the degree of \\ Master of Applied Science \\ in the program of
}

Mechanical and Industrial Engineering

Toronto, Ontario, Canada 2019

(C) Winsle Anpalagan, 2019 


\section{Author's Declaration}

I hereby declare that I am the sole author of this thesis. This is a true copy of the thesis, including any required final revision, as accepted by my examiners.

I authorize Ryerson University to lend this thesis to other institutions or individuals for the purpose of scholarly research.

I further authorize Ryerson University to reproduce this thesis by photocopying or by other means, in total or in part, at the request of other institutions or individuals for the purpose of scholarly research.

I understand that my thesis may be made electronically available to the public. 


\begin{abstract}
DEVELOPMENT OF AN OPTICAL METHOD TO MEASURE THE THERMAL CONDUCTIVITY OF TRANSPARENT NANOFLUIDS

Winsle Anpalagan
\end{abstract}

Master of Applied Science, 2019

Mechanical and Industrial Engineering

Ryerson University, Toronto

\begin{abstract}
A comparative technique to measure the thermal conductivity of nanofluids was developed using a Mach-Zehnder interferometer. The technique is based on one-dimensional heat transfer occurring through a layer of deionized water and a layer of nanofluid, separated by an aluminum barrier. The fluid layers were heated from above to produce thermal stratification and to minimize free convection. The temperature gradient at the surface of both fluid domains, where the heat transfer occurs by pure conduction, was measured optically. The model was designed and evaluated using computational fluid dynamics. An experimental model was fabricated, and preliminary experiments were conducted with a $\mathrm{SiO}_{2}$-water nanofluid. The results indicate that this comparative optical method is viable. However, the thin optical windows used in the current experiments made accurate measurements difficult, due to stress-induced bending of the optical windows. Recommendations for improvements in the model design are discussed.
\end{abstract}




\section{Acknowledgement}

I would like to acknowledge Dr. David Naylor and Dr. Tooraj Yousefi for their continuous support throughout my entire master's research. I am sincerely grateful for their immense knowledge, encouragement and patience till the end of the project. It was an honor to work with both influential professors, and I will forever cherish their teachings of what a truly enthusiastic and smart researcher should be like.

Besides my advisor, I am appreciative to Alan Machin for his time and assistance for providing constructive feedback and in building the experimental model. I would like to acknowledge Ryerson University for supporting this work and offering such a memorable opportunity.

I would like to thank my colleagues and friends for all the support they have given me, and for making my academic journey an enjoyable time. Lastly, I would like to thank my parents for their continuous love and support on such a challenging journey I had envisioned to fulfill. 


\section{Table of Contents}

AUTHOR'S DECLARATION

ABSTRACT

ACKNOWLEDGEMENT

LIST OF FIGURES $\quad$ x

LIST OF TABLES

LIST OF APPENDICES $\quad$ XV

NOMENCLATURE X xvii

CHAPTER 1: GENERAL REVIEW

1.1 Introduction 1

$\begin{array}{ll}\text { 1.2 Literature review } & 2\end{array}$

1.2.1 Nanofluids 2

1.2.2 Experimental techniques $\quad 5$

1.2.3 Empirical correlation equation for thermal conductivity of nanofluids 5

1.2.4 Comparing the correlation to experimental techniques $\quad 6$

1.2.5 Enhancement of convective heat transfer 9

1.3 Pure conduction through the horizontal surface 12

1.4 Scope of research $\quad 13$

CHAPTER 2: EXPERIMENTAL METHOD AND ANALYSIS 14 
2.1 Introduction $\quad 14$

2.2 Visibility of fringes $\quad 14$

2.3 Introduction to interferometry 19

2.3.1 The Mach-Zehnder interferometer 19

2.4 Interference fringe analysis $\quad 22$

2.4.1 MATLAB image processing code 24

2.5 Experimental model $\quad 25$

2.6 Thermal conductivity measurement 27

CHAPTER 3: EXPERIMENTAL APPARATUS AND PROCEDURE 29

$\begin{array}{ll}3.1 \text { Introduction } & 29\end{array}$

$\begin{array}{ll}3.2 \text { Experimental apparatus } & 29\end{array}$

3.2.1 Interferometer equipment 30

3.2.2 Calibration and leveling tools $\quad 31$

3.2.3 Temperature control units

3.2.4 Tools for fluid management 35

$\begin{array}{ll}\text { 3.2.5 Model } & 36\end{array}$

3.3 Experimental Procedure $\quad 39$

3.3.1 Sample preparation $\quad 39$

3.3.2 Model assembly $\quad 40$

$\begin{array}{ll}\text { 3.3.3 Experimental test run } & 41\end{array}$

3.3.4 Processing data 42

CHAPTER 4: NUMERICAL SIMULATION OF THE EXPERIMENTAL MODEL 43

$\begin{array}{lll}4.1 \text { Introduction } & 43\end{array}$ 
4.2 Model geometry $\quad 44$

4.3 Governing equations and boundary conditions 49

$\begin{array}{ll}\text { 4.3.1 Conservation of Mass } & 50\end{array}$

$\begin{array}{ll}\text { 4.3.2 Momentum equations } & 50\end{array}$

$\begin{array}{ll}\text { 4.3.3 Energy equation } & 51\end{array}$

$\begin{array}{ll}\text { 4.4 Temperature profile } & 51\end{array}$

4.5 Temperature distribution along the beam direction 55

4.6 Heat transfer rate at the barrier $\quad 58$

4.7 Time dependent solution $\quad 59$

CHAPTER 5: EXPERIMENTAL RESULTS AND ANALYSIS 61

$\begin{array}{ll}5.1 \text { Introduction } & 61\end{array}$

5.2 Preliminary experiment with nanofluid $\quad 61$

5.3 Effect of non-parallel optical windows $\quad 62$

5.4 Deflection in the optical window $\quad 65$

5.4.1 Approximation of the force $\quad 65$

$\begin{array}{ll}\text { 5.4.2 Deflection approximation } & 71\end{array}$

5.4.3 Comparison of simply supported beam solution to 3D FEA simulation 72

$\begin{array}{ll}5.5 \text { Water - water analysis } & 74\end{array}$

5.5.1 The subtraction method $\quad 75$

5.5.2 Finite fringe spacing and temperature difference $\quad 77$

$\begin{array}{ll}\text { 5.5.3 Experimental results } & 79\end{array}$

5.6 Water - nanofluid analysis $\quad 85$

5.6.1 Leakage issues $\quad 86$

5.6.2 Nanofluid results and comparison with data from the literature 87 
6.1 Conclusions $\quad 89$

$\begin{array}{ll}\text { 6.2 Recommendations } & 91\end{array}$

$\begin{array}{ll}\text { 6.2.1 Introduction } & 91\end{array}$

6.2.2 Recommendations for improvements to the experimental model 91

6.2.3 Recommendation for further research 94

APPENDIX A: WATER PROPERTIES 96

$\begin{array}{ll}\text { A.1 Thermal conductivity } & 96\end{array}$

$\begin{array}{ll}\text { A.2 Specific heat } & 97\end{array}$

$\begin{array}{ll}\text { A.3 Dynamic viscosity } & 98\end{array}$

$\begin{array}{ll}\text { A.4 Thermal diffusivity } & 99\end{array}$

$\begin{array}{ll}\text { APPENDIX B: EQUIPMENT } & 100\end{array}$

B.1 Specification of interferometer and equipment 100

APPENDIX C: MECHANICAL DRAWING OF THE MODEL 101

APPENDIX D: DETAILED EXPERIMENTAL PROCEDURE 109

$\begin{array}{ll}\text { Still image camera procedure } & 109\end{array}$

APPENDIX E: SAMPLE CALCULATION 111

$\begin{array}{ll}\text { E.1 Given data } & 111\end{array}$

E.2 Convert weight concentration to volume concentration 112

$\begin{array}{ll}\text { E.3 Nanofluid sample preparation } & 112\end{array}$

E.4 Predict the total fringe shift 113

E.5 Surface temperature (temperature of the barrier) 113 
E.6 Extracting fringe gradient from scanning algorithm

E.7 Subtraction technique

Finite fringe setting

Infinite fringe setting

E.8 Relative thermal conductivity of the test fluid

F.1 Uncertainty in the absolute thermal conductivity

G.1 Thermocouple calibrations 


\section{List of Figures}

Figure 1.1: Comparison between correlations vs experimental data on the relative thermal conductivity of $\mathrm{SiO}_{2}$-water nanofluid up to $5 \%$ by volume concentration at $20^{\circ} \mathrm{C}$. 8

$\begin{array}{ll}\text { Figure 1.2: An idealized configuration of the experimental configuration. } & 12\end{array}$

Figure 2.1: Infinite fringe interferogram for $\mathrm{Al}_{2} \mathrm{O}_{3}$-water nanofluid; $0.1 \% \mathrm{wt}$ after homogenization (Yousefi et al., 2015).

Figure 2.2: Infinite fringe interferogram for $\mathrm{SiO}_{2}$ - water nanofluid; $0.07 \%$ wt. (Yousefi et al., 2015).

Figure 2.3: Experiment performed with $\mathrm{SiO}_{2}$-water nanofluid at $10.1 \%$ by volume concentration using finite fringe setting.

Figure 2.4: Temperature coefficient of refractive index at varying temperatures and weight concentrations with $\mathrm{SiO}_{2}$-water nanofluid (Yousefi, 2019).

Figure 2.5: Setup of the Mach-Zehnder Interferometer.

Figure 2.6: Isometric view of the experimental model.

Figure 3.1: Apparatus of the experiment.

Figure 3.2: Frame design with the model tightened in position.

Figure 3.3: Full view of the frame within the interferometry setup.

Figure 3.4: CPU cooling units with modifications to connect to the water bath (front and top view)

Figure 3.5: Half of the model assembled, without the O-rings in place.

Figure 3.6: Isometric view of the optical frame. 
Figure 4.1: Sketch of the 2D model: (1) aluminum thin barrier, (2) \& (3) water fluid domains, (4) cold plate, (5) hot plate, and (6) housing. , and (7) insulation.

Figure 4.2: 3D model in SolidWorks.

Figure 4.3: Reduced computational domain due to symmetric geometry and boundary conditions. The shaded section is the computational domain.

Figure 4.4: Two-dimensional temperature profile along the center line of the enclosure.

Figure 4.5: Vertical temperature profile on the center line of the model from 3-D study.

Figure 4.6: Vertical temperature profile near the barrier from 3-D study.

Figure 4.7: Velocity vectors and streamline superimposed over the temperature field from 3-D study.

Figure 4.8: Cross section of the experimental model showing the line segments where the temperature variation in the beam direction has been plotted.

Figure 4.9: Temperature distribution in the beam direction at $3 \mathrm{~mm}$ away from the datum $\mathrm{x}$-axis

Figure 4.10: Time dependent solution of the temperature distribution in the experimental model from the datum. Temperatures are on the vertical center line of the model.

Figure 5.1: Finite fringe image of the unheated model with $\mathrm{SiO}_{2}$ - water nanofluid (10\% volume concentration) and water.

Figure 5.2: Misaligned optical windows.

Figure 5.3: Unheated reference image in infinite fringe mode with water in both fluid domains.66

Figure 5.4: Plot of the pixel intensity with distance from the top of the upper fluid domain (for image shown in Fig. 5.3). 
Figure 5.5: Optical window bending due to deflection (left) \& simply supported beam with concentrated load at the center (right).

Figure 5.6: Rectangular geometry corresponding to the moment of inertia equation.

Figure 5.7: Compressive force (per unit circumference) versus compressive strain the silicon Oring (1.6 mm dimeter O-ring) (Parker, 2019).

Figure 5.8: Line graph that demonstrates the number of fringes and fringe gradient caused by the deflection on the optical window.

Figure 5.9: Optical window deflection simulation analysis performed on SolidWorks.

Figure 5.10: Finite fringe setting with $6^{\circ} \mathrm{C}$ temperature difference, a) unheated model, b) heated model.

Figure 5.11: Finite fringe setting with $10^{\circ} \mathrm{C}$ temperature gradient, a) unheated model, b) heated model.

Figure 5.12: Experiment performed in finite fringe mode with deionized water in both fluid domains at a temperature difference of $10^{\circ} \mathrm{C}$ with the barrier temperature set at $20^{\circ} \mathrm{C}$, a) unheated model, b) heated model.

Figure 5.13: Fringe gradient due to the temperature field at the barrier, extracted from the images shown in Figure 5.12.

Figure 5.14: Experiment performed in infinite fringe mode with deionized water in both fluid domains at a temperature difference of $10^{\circ} \mathrm{C}$ with the barrier temperature set at $20^{\circ} \mathrm{C}$, a) unheated mode, b) heated model.

Figure 5.15: Fringe gradient at the barrier due to temperature, measured with water in both fluid domains in infinite setting mode shown in Figure 5.14.

Figure 5.16: Absolute thermal conductivity of water at $20^{\circ} \mathrm{C}$ measured using finite and infinite fringe settings. 
Figure 5.17: Shadowgraph images of the unheated model with deionized water in the upper domain and $10 \% \mathrm{SiO}_{2}$-water nanofluid (by volume) in the lower domain.

Figure 5.18: Experiment performed with $\mathrm{SiO}_{2}$-water at $10.1 \%$ by volume concentration with a temperature gradient at $10^{\circ} \mathrm{C}$, a) unheated model, b) heated model.

Figure 5.19: Comparing the relative thermal conductivity measurements performed with $\mathrm{SiO}_{2}-$ water at $20^{\circ} \mathrm{C}$ in finite fringe setting mode to correlations discussed in the literature review. 


\section{List of Tables}

Table 1.1: Comparison of convective heat transfer enhancement for various flow conditions and geometries.

Table 4.1: Material properties.

Table 4.2: Boundary conditions applied.

Table 4.3: Percent difference in predicting the lower fluid domain's thermal conductivity at $20^{\circ} \mathrm{C}$.

Table 4.4: Fluid temperature distribution in the beam direction at varying $\mathrm{x}$-locations from the thin barrier.

Table 4.5: Heat transfer rate at the barrier. 


\section{List of Appendices}

\section{List of Figures in Appendices}

Figure C.1: Aluminum barrier.

Figure C.2: Delrin side block (with thermocouples).

Figure C.3: Delrin side block (without thermocouple).

Figure C.4: Aluminum hot/cold block.

Figure C.5: Delrin optical frame (front).

Figure C.6: Delrin optical frame (back).

\section{List of Tables in Appendices}

Table A.1: Thermal conductivity of water at varying temperatures.

Table A.2: Specific heat of water at varying temperatures.

Table A.3: Dynamic viscosity of water at varying temperatures.

Table B.1: Specification of the optical component in the interferometer.

Table C.1: Bill of material for the model.

Table C.2: Fasteners used to assemble the model.

Table F.1: Summary of sources of uncertainty.

Table G.1: Hot plate thermocouple calibration.

Table G.2: Data gathered from the scanning algorithm performed in finite mode with water in both fluid domains. 
Table G.3: Inversed measured data gathered from the scanning algorithm performed in finite fringe mode with water in both fluid domains.

Table G.4: Measured fringe gradient data with water to water experiment performed in finite fringe mode.

Table G.5: Fringe gradient of the measured data for both water fluid domains using subtraction technique.

Table G.6: Data gathered from the scanning algorithm.

Table G.7: Measured fringe gradient data with water to water experiment.

Table G.8: Fringe gradient of the measured data for both fluid domains using subtraction technique.

Table G.9: Data gathered with experimented performed with $\mathrm{SiO} 2-$ water nanofluid at $7.1 \%$ by volume concentration in finite fringe mode.

Table G.10: Data gathered with experimented performed with $\mathrm{SiO} 2$-water nanofluid at $10 \%$ by volume concentration in finite fringe mode. 


\section{Nomenclature}

A

c

$\mathrm{c}_{\mathrm{o}}$

$\mathrm{c}_{\mathrm{p}}$

E

$\mathrm{F}_{\text {ga }}$

g

ga

$\mathrm{H}$

$\mathrm{h}$

I

$\mathrm{k}$

L

1

$\mathrm{N}$

n

$\mathrm{Nu}$

$\dot{Q}$

$\dot{q}$

$\mathrm{Ra}$

$\mathrm{SF}_{\mathrm{v}}$

$\mathrm{T}$

$\mathrm{t}$

TC

th

$u, v, w$

$\overrightarrow{\mathrm{V}}$ area of the specified cavity $\left[\mathrm{m}^{2}\right]$

speed of light [m/s]

speed of light in a vacuum [m/s]

specific heat $[\mathrm{J} / \mathrm{kg} \cdot \mathrm{K}]$

modulus of elasticity $\left[\mathrm{N} / \mathrm{m}^{2}\right]$

compressive force from gasket [N]

gravitational acceleration $\left[\mathrm{m} / \mathrm{s}^{2}\right]$

represents the gasket/ O-ring

height of the fluid domain [m]

heat transfer coefficient $\left[\mathrm{W} / \mathrm{m}^{2} \cdot \mathrm{K}\right]$

Moment of inertia $\left[\mathrm{m}^{4}\right]$

thermal conductivity $[\mathrm{W} / \mathrm{m} \cdot \mathrm{K}]$

path length of the beam through the fluid [m]

length of the optical window [m]

number of wavelengths

index of refraction

Nusselt number

Heat transfer rate [W]

heat flux $\left[\mathrm{W} / \mathrm{m}^{2}\right]$

Rayleigh number

scale factor in the vertical direction [m/pixel]

temperature $[\mathrm{K}]$

refers to time $[\mathrm{s}]$

thermocouple [K]

Thickness of specified cavity [m]

velocity components

Vector velocity field 
W

$\mathrm{X}$

$\mathrm{X}, \mathrm{Y}, \mathrm{Z}$

$\mathrm{Z}_{\mathrm{d}}$

Greek Symbols

$\alpha$

$\Delta \mathrm{s}$

$\Delta \varepsilon$

$\varepsilon$

$\theta$

$\lambda$

$\lambda_{0}$

$\mu$

$\omega$

$\rho$

$\phi$

$\varphi$

Subscript

$\infty$

b

bf

c

$\mathrm{h}$ optical window thickness [m]

fringe distance $[\mathrm{mm}]$

Cartesian coordinate system, [m]

maximum deflection in the optical window $[\mathrm{m}]$

thermal diffusivity $\left[\mathrm{m}^{2} / \mathrm{s}\right]$
additional path length $[\mathrm{m}]$
change in the fringe order $[\mathrm{m}]$
Fringe shift order
Offset angle between parallel optical windows(degrees)
wavelength $[\mathrm{m}]$
vacuum wavelength $[\mathrm{m}]$
dynamic viscosity $[\mathrm{N} \cdot \mathrm{s} / \mathrm{m}]$
weight concentration $(\%)$
density $\left[\mathrm{kg} / \mathrm{m}^{3}\right]$
volume concentration $[\%]$
dissipation function




$\begin{array}{ll}\mathrm{nf} & \text { any type of transparent nanofluid } \\ \mathrm{np} & \text { value for the nanoparticles } \\ \mathrm{o} & \text { initial value } \\ \mathrm{ref} & \text { value for the reference beam } \\ \mathrm{S} & \text { value for the surface of the thin barrier } \\ \mathrm{S}_{\mathrm{c}} & \text { correction due to deflection } \\ \text { test } & \text { value for the test beam } \\ \mathrm{tf} & \text { refers to test fluid in the model } \\ \mathrm{W} & \text { value for the water }\end{array}$

Abbreviations

bf

BS

CFD

CPU

FEA finite element analysis

FVM finite volume method

MZI

ref

TC

test

tf

vc

wt base fluid

beam splitter

computational fluid dynamics

central processing unit

Mach-Zehnder interferometer

reference beam

thermocouple

test beam

test fluid

volume concentration

weight concentration 


\section{Chapter 1: General Review}

\subsection{Introduction}

For many years, researchers in the heat transfer have continuously strived to find new opportunities to enhance the heat transfer in engineered applications. The problem was approached by exploring new techniques, new geometric structures or even new materials. One approach that has become of interest to many researchers for over a decade is nanofluids. In this application, nanoparticles are mixed with a base fluid, for the purpose of improving the properties of the fluid, particularly thermal conductivity. The purpose of the current study is to develop an optical method to measure the thermal conductivity of nanofluids.

Nanofluids have been researched for decades, and continue to be studied (Cengel, 2014). Nanofluids are a mixture of a base fluid and nano-sized particles of a specific metal oxides. The metal oxides in the fluids are known to be good conductors. Several studies have examined the property changes due to the influence on specific parameters of the fluid. For thermal conductivity of the fluid, there are four specific parameters that have been shown to have a significant influence: the material, shape, size of the nanoparticles and the volume concentration of the nanofluid (Choi \& Jeffrey, 1995). The current study will investigate the effect of volume concentration of silicon dioxide $\left(\mathrm{SiO}_{2}\right)$.

The specific nanofluids that will be used in the experiment will be $\mathrm{SiO}_{2}$ with water. The selected nanofluids are suitable for the experimental design, as a transparent fluid is required for the optical technique used for the study. The transparency of the fluid will allow visualization of the temperature field using a laser interferometry. As the volume concentration of the nanoparticles increase, the difficulty in achieving visible interference fringes increases correspondingly (Yousefi et al., 2015).

The current study will involve developing an optical method to measure the thermal conductivity of nanofluids experimentally. Several researchers have performed experimental studies on the potential enhancement to the heat transfer coefficient using nanofluids (e.g. Rao \& Atul, 2014; Haridas et al., 2015). However, those studies have relied on the data of thermal conductivity of the 
corresponding nanofluid commonly from an empirical equation. The empirical equation is the basis of several experimental intrusive techniques that have measured the thermal conductivity of various nanofluids. It was apparent that a reliable method to measure the thermal conductivity was needed. Thus, the current study will be the first to use interferometry to measure the thermal conductivity of nanofluids.

In summary, the current study will involve developing an optical method to measure the thermal conductivity of transparent nanofluids experimentally. $\mathrm{A} \mathrm{SiO}_{2}$-water nanofluid was used in this thesis. A Mach-Zehnder interferometry will be used to measure the temperature gradient at the surface where conduction is the dominant heat transfer mode. The technique will also be modelled using computational fluid dynamics (CFD) to assess the adequacy of the experimental design.

\subsection{Literature review}

The development of an optical method to measure the thermal conductivity of $\mathrm{SiO}_{2}$-water nanofluids is the focus of the study. The basis of the existing techniques to measure thermal conductivity will be discussed. As well, a review of the investigated heat transfer enhancements achieved using nanofluids will be included.

\subsubsection{Nanofluids}

As mentioned previously, nanofluids have been researched for some time. The interest of researchers investigating nanofluids was to understand the significant enhancement in heat transfer rates with varying concentration of nanoparticles in base fluids. The advantages of nanofluid is based on the interaction of the molecular structure of the metal ions containing a sea of electrons moving freely along the surface of the ions, influencing the particle movement. This enhancement of the particle movement with both solid and fluid particles in a medium increases the particle collision rate, which leads to an increase in the transfer of heat energy. The collision of the particles influencing the heat transfer is defined as the thermal conductivity (Cengel, 2014). By combining the metal ions in an aqueous solution like water (base fluid), the properties are expected to have a 
positive effect on the thermal conductivity. However, scientific data is required to understand the magnitude of the changes to the thermal conductivity of nanofluids.

There have been several experimental and theoretical examinations to explore the thermal performance of the nanofluids. From the published data on nanofluids, there are several notable parameters that contribute to the enhancement of the thermal conductivity of the nanofluids (Liu $\&$ Chen, 2014). The particle size, shape, material and the volume concentration of the nanoparticles each have a role in improving the nanofluid's thermal conductivity (Liu \& Chen, 2014). From observation of the documented results, increasing the concentration of nanoparticles is expected to cause an increase in the thermal conductivity. However, even a $0.3 \%$ volume concentration of the copper nanoparticles in ethylene glycol has shown an enhancement of $40 \%$ in the thermal conductivity compared to the base fluid (Eastman et al., 2001).

Aside from the notable parameters that have been shown to improve the thermal conductivity, there has been debate over other factors that have contributed to the improvement of the thermal conductivity. Brownian motion is believed to be one of the contributing factors that influence the thermal conductivity of the fluid. In short, Brownian motion is the agitation of the particles, which changes with respect to the temperature. The changes to the agitation of the particles affects the heat transport of each individual particle within the medium. For example, a particle subjected to a cold environment distributes the kinetic energy much more slowly to its surrounding particles than a particle in a hotter environment (Rao et al, 2014; Wang et al., 1999).

Another commonly discussed mechanism for improvements in the measured thermal conductivity is the effect of thermophoresis. As discussed by a few researchers in experimental studies [Tijerina et al., 2018; Ryzhkov et al.,2014], thermophoresis has been found to have a significant effect on the thermal conductivity of nanofluid solutions. Thermophoresis can be explained by observing the particle migrations of the nanoparticles in the base fluid. The particle migration varies depending on the size and density of the particle under the influence of the temperature gradient. The nanoparticles are usually heavier than the base fluid and will migrate from the hot to cold area. However, the response of the particle migration is different in a natural and forced convection apparatus. The effects of mixing of the nanofluid are greater in forced convection than in natural convection due to the fluid flow and effects like eddies. A study that investigated the effect of thermophoresis has shown significant changes in the local volume concentration of a nanofluid in 
a natural convective setting. The study was done by measuring 14 different locations along the surface and found higher concentrations near the colder surface [Khalili et al, 2017].

Equally important, non-separated nanoparticles have an impact on the enhancement of thermal conductivity. One preparation technique includes a coating agent with the nanoparticles. The purpose of the coating agent is to ensure that the nanoparticles are separated from each other when they are mixed with the base fluid. Even though the mixing process takes hours to yield a stable solution, there is a chance that a few nanoparticles will bond with each other. Non-separated particles develop a structure similar to chainlike bonding, which can change the behavior of the particle motion. A particle with a chain structure can transport more heat along the direction of heat flux (Hamilton \& Crosser, 1962).

As mentioned in a previous paragraph, volume concentration is one of the critical factors that can influence thermal conductivity. Research have shown that the thermal conductivity of nanofluid significantly increase with increasing particle volume concentration. Xie et al. (2011) documented the thermal conductivity enhancement of varying concentrations with graphene, oxide and nonoxide nanofluids, all of which have shown a similar trend at very low concentration. A volume concentration of $0.05 \%$ has been shown to have a $60 \%$ enhancement. Concentrations between 20 to $40 \%$ have shown an enhancement in the thermal conductivity, but equal to the enhancement present at lower concentrations (Kakac \& Pramuanjaroenkij, 2009). The reason many of the studies that analyzed the thermal conductivity of nanofluids considered low concentrations is due to the dramatic enhancement in thermal conductivity.

Another concern for the current study is the calculation of the volume concentration of the experimented nanofluid. The nanofluid used in the present experiment will be a commercial nanofluid, purchased from MkNANO. The undiluted water-based nanofluids that will be used in this study are $\mathrm{SiO}_{2}$ at $40 \%$ by weight concentration with particle size of $20 \mathrm{~nm}$. In order to compare our experimental data with other literature, it is required to convert the weight percent to a volume concentration. Azmi et al. (2013) has provided an equation to convert the weight percent of the nanoparticles to a volume concentration, based on density of water $\left(\rho_{\mathrm{w}}\right)$ and nanoparticles $\left(\rho_{\mathrm{np}}\right)$. 


\subsubsection{Experimental techniques}

Since the 1990's, measurements of the thermal conductivity of nanofluids have been performed using intrusive measuring techniques. For instance, a common intrusive technique is the transient hot wire method. The measuring principle is based on the calculation of the transient temperature profile around a thin wire. The thin wire is the line source which is supplied with a constant heat flux by monitoring the current supplied to the wire. The technique is popular, as it is considered an accurate measurement method, even though it is an intrusive technique. From the research of Murshed et al. (2005), the accuracy of the measurement technique was estimated to be $2 \%$ using comparisons to the thermal conductivity of distilled water.

Another common intrusive measurement technique to measure the thermal conductivity of a nanofluids using a thermocouple at steady state. Wang et al. (1999) used a one-dimensional steady state parallel plate method to measure the temperature with 14 thermocouples along the direction that the heat flux was applied. The experiment was performed with an $\mathrm{Al}_{2} \mathrm{O}_{3}$-water nanofluid. The thermal conductivity was enhanced by $16 \%$ for $5.5 \%$ volume concentration of alumina when compared to water (Wang et al., 1999).

\subsubsection{Empirical correlation equation for thermal conductivity of nanofluids}

Researchers that are interested to understand the potential of nanofluids in heat transfer applications need to be able to predict the thermal conductivity of the nanofluids for varying concentrations and temperatures. A large set of the available measurements of the thermal conductivity were plotted on a scatter plot by Sharma et al. (2012). Sharma et al. (2012) developed a regression analysis of 252 data for a wide range of nanofluids, e.g. Copper $(\mathrm{Cu})$, Copper Oxide $(\mathrm{CuO})$, Titanium Dioxide $\left(\mathrm{TiO}_{2}\right)$, Silicon Carbide $(\mathrm{SiC})$, Zirconium Dioxide $\left(\mathrm{ZrO}_{2}\right)$, and Alumina $\left(\mathrm{Al}_{2} \mathrm{O}_{3}\right)$. The regression analysis gave an empirical equation with a standard deviation of $3.8 \%$ and a marginal error of $\pm 10 \%$, which can be used to predict the thermal conductivity of various waterbased nanofluids. Equation (1.1) is Sharma's correlation equation used to predict the thermal conductivity of nanofluids. 


$$
\begin{gathered}
k_{n f}=k_{w}\left[0.8938\left(1+\frac{\phi}{100}\right)^{1.37} \times\left(1+\frac{T_{n f}}{70}\right)^{0.2777} \times\left(1+\frac{\rho_{n p}}{150}\right)^{-0.0366} \times\right. \\
\left.\left(1+\frac{\alpha_{n p}}{\alpha_{w}}\right)^{0.01737}\right]
\end{gathered}
$$

where $\phi$ is the volume fraction, $\mathrm{T}_{\mathrm{nf}}$ is the temperature $\left({ }^{\circ} \mathrm{C}\right), \rho_{n p}$ is the nanoparticle density, and $\frac{\alpha_{n p}}{\alpha_{w}}$ is the particle to water thermal diffusivity ratio.

However, one issue with the empirical method for predicting the thermal conductivity is that it requires the density and thermal diffusivity of the nanoparticle. For commercial nanofluids, obtaining both parameters is difficult, as the companies producing the nanoparticles do not generally supply this information. Thus, the additional equations provided by Sharma equations for predicting the density and thermal diffusivity would amplify the uncertainty in predicting the thermal conductivity from the empirical equation (1.1).

In addition, an issue which revolves around intrusive techniques is the level of uncertainty associated when measuring the thermal conductivity. For the hot wire method, the drawbacks of the measurement are the buoyancy-driven convection, which disturbs the measurements. Techniques that depend on thermocouples also have errors. The first issue is that the physical probes disturb the temperature distribution. This is because of the probes conduct heat away from/to the immersed fluid. As well, the probes would gain/lose heat from the surrounding due to conduction, radiation or convection, thus affecting the accuracy of the thermocouple measurement. Based on a review of the literature, the optical method proposed in the current study is the first to measure the thermal conductivity of nanofluids with a non-intrusive technique. With an interferometer, the level of uncertainty compared to the intrusive technique may be lower. The interferometry can provide a measurement of the temperature profile without disturbing the physical phenomena of the experiment.

\subsubsection{Comparing the correlation to experimental techniques}

The reliability of the several correlations were analyzed by comparing the experimental data for $\mathrm{SiO}_{2}$-water nanofluids with the correlation at low volume concentrations. Comparison of the correlation to measured data from recent studies provides an indication of the reliability of the 
correlation. It is important to note that Sharma's correlations did not include any experimental data involving $\mathrm{SiO}_{2}$-water in the regression analysis but was still used by many researches for experiments involving $\mathrm{SiO}_{2}$-water nanofluid. Figure 1.1 demonstrates the comparison of the experimental research as previously discussed to Sharma's correlation up to $5 \%$ by volume concentration. The experimental data that were used in the comparison are a combination of intrusive and non-intrusive measurements of $\mathrm{SiO}_{2}$-water nanofluids with varying sizes, shapes, and volume concentrations. For the Sharma correlation, one error that can be identified is the prediction of a relative thermal conductivity lower than 1 for concentrations below $1.6 \%$. Thus, the correlation fails to predict the property of water alone. It is important to note that many of the existing correlations have performed poorly to predict the thermal conductivity for $\mathrm{SiO}_{2}$-water nanofluid compared to the experimental data. This demonstrates that there is room for improvement in predicting the thermal conductivity of nanofluids. 


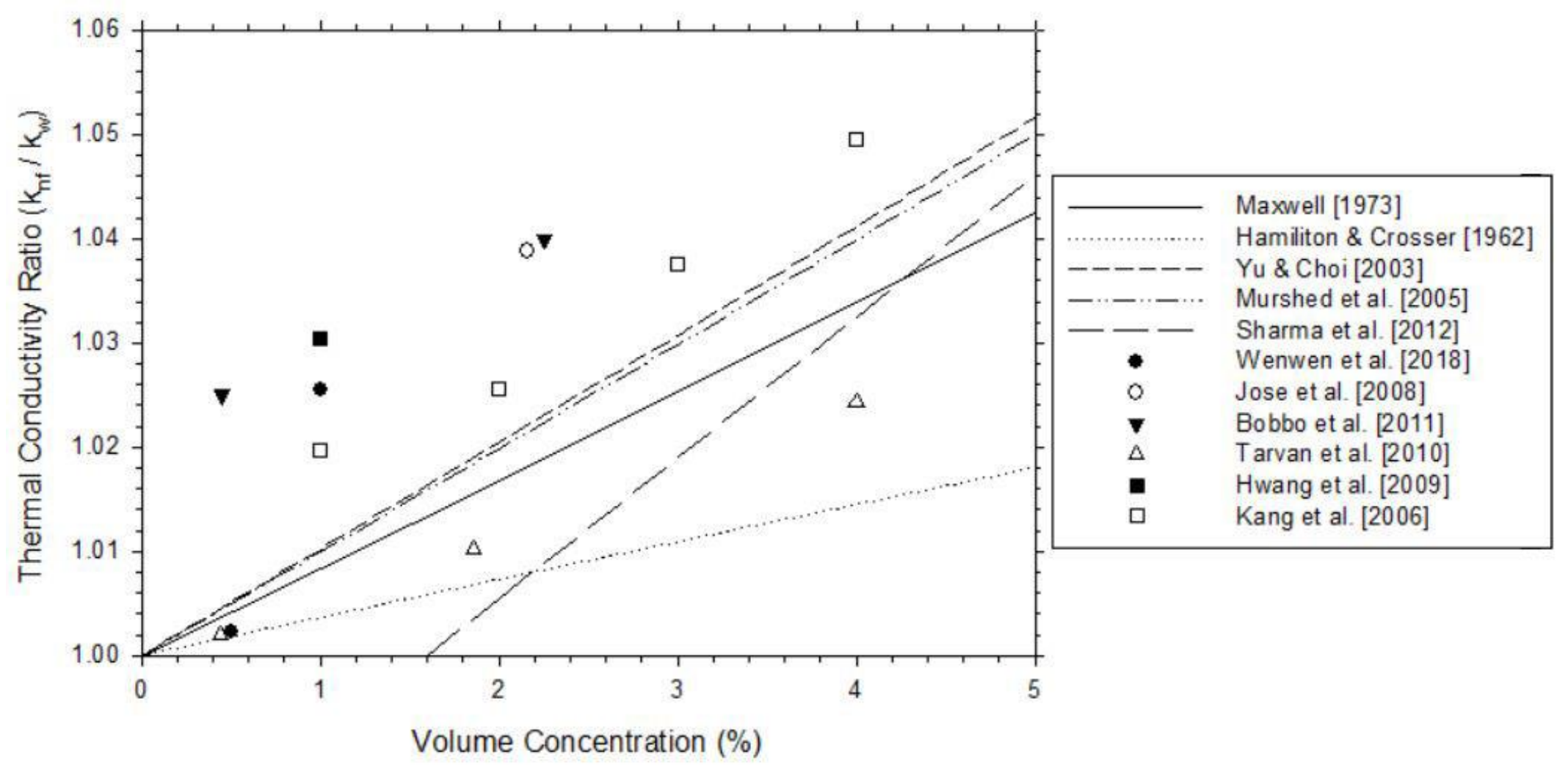

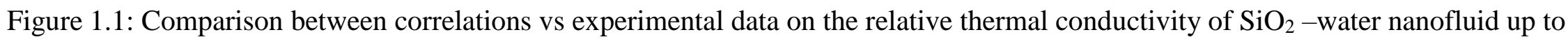
$5 \%$ by volume concentration at $20^{\circ} \mathrm{C}$. 


\subsubsection{Enhancement of convective heat transfer}

The advantages of improving the thermal conductivity of a base fluid with nanoparticles introduces the possibility of enhancing the heat transfer overall. For this reason, researchers were curious to determine the capability of improving the thermal conductivity of the nanofluids and the corresponding changes to the heat transfer coefficient. Many recent papers have investigated the percentage of enhancement of the heat transfer coefficient in different convective experiments.

There are several studies that have investigated the enhancement of the heat transfer with intrusive technique such as transient wire method (Buongiorno et al., 2009), temperature oscillation method (Das et al., 2003) and 3- $\omega$ method (Choi, 2009). However, the following discussion will consider the enhancement of the heat transfer that was investigated with an interferometer. The purpose is to compare the levels of heat transfer enhancement obtained in previous studies using the interferometric technique.

One reason to study the previous work in this area is to consider possible improvements that can be made to the current experimental technique for optically measuring the thermal conductivity of nanofluids. In the measurements of Rao et al. (2014), it was shown that a $21 \%$ and $13 \%$ enhancement to the heat transfer was apparent with just $0.02 \%$ and $0.01 \%$ volume concentration of $\mathrm{Al}_{2} \mathrm{O}_{3}$ respectively. The experiment was performed by observing the temperature profile of a natural convection setup where the nanofluid was heated by a vertical plate.

In the research of Haridas et al. (2015), an experiment was performed in a forced convection in a round pipe for a range of Reynolds number. The results have shown an $8 \%$ increase to the heat transfer with 3\% volume concentration of $\mathrm{Al}_{2} \mathrm{O}_{3}$-water. As well, Rajput et al. (2016) has shown up to $30 \%$ increase to the heat transfer coefficient at $0.01 \%$ volume concentration of $\mathrm{Al}_{2} \mathrm{O}_{3}$-water. When the volume concentration was increased by $0.02 \%$ increments up to $0.05 \%$, the percentage enhancement to the heat transfer coefficient decreased by a $1-3 \%$.

Table 1.1 summaries the results of several studies of the enhancement of convective heat transfer using water-based nanofluids. All of these studies have shown a drastic enhancement of the convective heat transfer at very low nanoparticle concentrations. Some of the reason behind the fluctuation is the uncertainty in the thermal conductivity used in the Nusselt number calculation. 
Table 1.1: Comparison of convective heat transfer enhancement for various flow conditions and geometries.

\begin{tabular}{|c|c|c|c|c|}
\hline Authors & Flow Geometry & Nanofluid & $\begin{array}{l}\text { Volume } \\
\text { Conc. } \\
\phi(\%)\end{array}$ & $\begin{array}{c}\text { Enhancement compared to } \\
\text { the base fluid }\end{array}$ \\
\hline $\begin{array}{l}\text { Wen and Ding } \\
\text { [2004] }\end{array}$ & $\begin{array}{l}\text { Laminar flow in } \\
\text { a constant heat } \\
\text { flux tube }\end{array}$ & $\mathrm{Al}_{2} \mathrm{O}_{3}$-water & 1.6 & $\begin{array}{l}41 \% \text { and } 47 \% \text { increase to } \\
\text { the heat transfer coefficient } \\
\text { at } \mathrm{Re}=1050 \text { and } 1600 \\
\text { respectively }\end{array}$ \\
\hline $\begin{array}{l}\text { Xuan and Li } \\
\text { [2003] }\end{array}$ & $\begin{array}{l}\text { Turbulent flow } \\
\text { through an } \\
\text { isothermal tube }\end{array}$ & $\mathrm{Cu}$-water & 2 & $39 \%$ in the Nusselt number \\
\hline $\begin{array}{l}\text { Hwang et al. } \\
\text { [2009] }\end{array}$ & $\begin{array}{l}\text { Uniformed } \\
\text { heated circular } \\
\text { tube in the fully } \\
\text { developed } \\
\text { laminar flow } \\
\text { regime }\end{array}$ & $\mathrm{Al}_{2} \mathrm{O}_{3}$-water & 3 & $\begin{array}{l}8 \% \text { to the heat transfer } \\
\text { coefficient }\end{array}$ \\
\hline $\begin{array}{l}\text { Chandrasekar } \\
\text { et al. [2010] }\end{array}$ & $\begin{array}{l}\text { Circular pipe } \\
\text { under laminar } \\
\text { flow with wire } \\
\text { coil inserts }\end{array}$ & $\mathrm{Al}_{2} \mathrm{O}_{3}$-water & 0.1 & $\begin{array}{l}\text { Increased Nusselt number } \\
\text { by } 12.24 \% \text { at } R e=2275\end{array}$ \\
\hline $\begin{array}{l}\text { Nguyen et al. } \\
\text { [2007] }\end{array}$ & $\begin{array}{l}\text { Closed fluidic } \\
\text { system operated } \\
\text { with turbulent } \\
\text { flow regime }\end{array}$ & $\mathrm{Al}_{2} \mathrm{O}_{3}$-water & 6.8 & $\begin{array}{l}40 \% \text { to the heat transfer } \\
\text { coefficient }\end{array}$ \\
\hline
\end{tabular}


The problem with the published data that have investigated the heat transfer coefficient of nanofluids obtained using interferometry is the uncertainty in two important fluid properties. The properties are the thermal conductivity of the nanofluids, and the temperature coefficient of refractive index $(\mathrm{dn} / \mathrm{dT})$. Uncertainty in these properties each contribute to the uncertainty of the measurement technique.

The temperature coefficient of refractive index is an optical property that is not fully considered in many of the research papers. Many previous studies have neglected changes in this optical property at low nanoparticle concentrations. The assumption that the temperature coefficient of refractive index is equal to that of the base fluid (water) could critically jeopardize the results. One of the objectives of the current experiment is to minimize the level of the uncertainty as much as possible. For the current study, the data on the temperature coefficient of $\mathrm{SiO}_{2}$-water nanofluid will be based on the measurements of Yousefi et al. (2019) data. Yousefi et al. (2019) have recently measured the thermal coefficient of refractive index over a range of volume concentrations and fluid temperatures with an interferometer.

An idealized layout of the current experimental method is shown in Figure 1.2. The model will be constructed to obtain nearly one-dimensional heat transfer occurring through a layer of nanofluid and a layer of pure water, separated by a thin aluminum plate. The fluid layers will be heated from above to produce thermal stratification and minimize free convection in both fluid domains. The optically-measured temperature gradient at the aluminum plate surface will be used to obtain the thermal conductivity of the nanofluid relative to that of the pure deionized water. Since there are two fluid domains, the data on $\mathrm{dn} / \mathrm{dT}$ will be crucial to optically predict the thermal conductivity of the nanofluid. By using the data of Yousefi et al. (2019) for the temperature coefficient of refractive index, the goal is to obtain a more accurate measurement of the thermal conductivity of the nanofluid. 


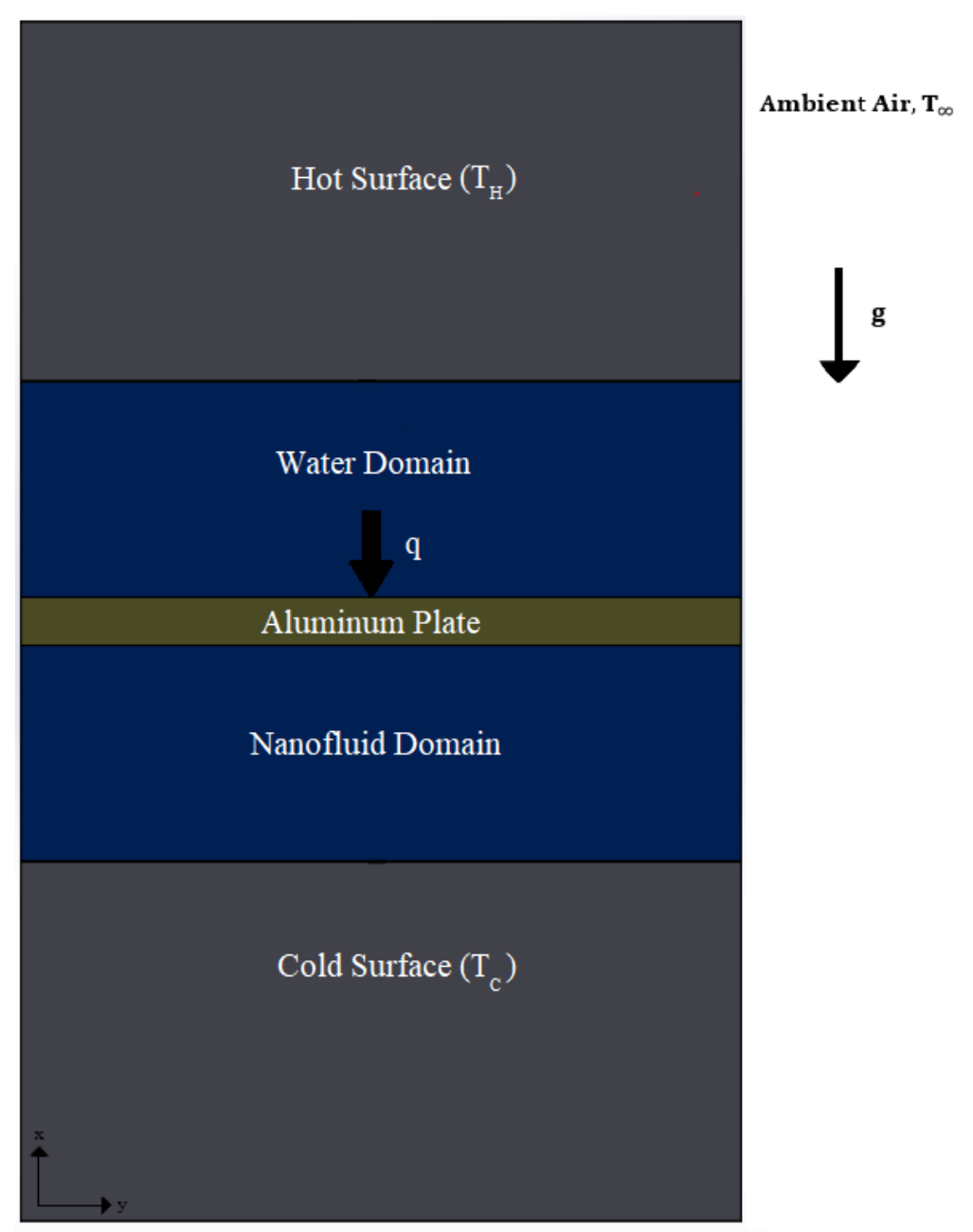

Figure 1.2: An idealized configuration of the experimental configuration.

\subsection{Pure conduction through the horizontal surface}

The premise of the measurement technique is to measure the thermal conductivity is based on Fourier's law, which is represented in equation (1.2). In Eq. (1.2), $\dot{q}$ is the heat flux (W/m²), $\mathrm{k}$ is the thermal conductivity of the fluid, and $\mathrm{dT} / \mathrm{dx}$ is the temperature gradient.

$$
\frac{\dot{Q}}{A}=\dot{q}=-k \frac{d T}{d x}
$$

Eq. (1.2) will be applied at the surface of the thin plate between both fluid layers. At the solid surface the heat transfer occurs by pure conduction due to the no-slip condition. 
The objective of the research is to develop an optical method to measure the thermal conductivity of transparent nanofluids for varying volume concentrations and temperatures. Prior to the experiment, a numerical solution will be obtained to validate the technique and identify the possible problems to overcome in the experimental model design. The particular concern is to make sure the heat transfer through the barrier between the two fluids is sufficiently onedimensional. In particular, the heat losses to the optical windows of the experimental model need to be assessed. A Mach-Zehnder interferometer will be used to visualize the temperature profile in order to determine the thermal conductivity of the nanofluid.

As previously mentioned, a numerical solution of the heat transfer in the experimental model will be presented. The purpose of numerically simulating the experimental model is to assess the accuracy of the proposed technique. Several test will be performed, to illustrate that the proposed method is viable. The computation fluid dynamics (CFD) module of the commercial software SolidWorks will be used to numerically simulate this problem.

\subsection{Scope of research}

In summary, the objective of the research is to develop an optical method to measure the thermal conductivity of transparent nanofluids as a function of volume concentration and temperature. The fluids used in this research will be deionized water and a water-based $\mathrm{SiO}_{2}$ nanofluid. The measurements will be performed using a Mach-Zehnder interferometer. This optical instrument will be used to measure the temperature profile, which will be used to determine the thermal conductivity of the nanofluid relative to water. Before the experiments are conducted, a numerical simulation of the experimental model will be obtained to identify design issues and to validate the method.

The technique that will be developed in this thesis is intended for the measurement of the thermal conductivity of nanofluids. However, the method is also applicable to measure the thermal conductivity of any other fluids that are transparent. It is essential to have a reliable experimental technique to measure the thermal conductivity of nanofluids before extending this research to optical measurements of convective heat transfer enhancement. In convection studies, the fluid's thermal conductivity is needed to evaluate both the Nusselt number and the Prandtl number. 


\section{Chapter 2: Experimental Method and Analysis}

\subsection{Introduction}

This chapter will cover the details of interferometry and its application to the study. The MachZehnder interferometer will be the measurement tool used to determine the thermal conductivity of the nanofluid. The content will provide an overview of each component and the layout required for a Mach-Zehnder interferometry. In sequential order, the methodology of the techniques to measure the thermal conductivity will be explained. This discussion starts with an explanation of how the interferometer produces interference fringes. The number of fringes produced by the laser path passing through the experimental model is presented. This is followed by a description of how the temperature gradient is measured to calculate the thermal conductivity of the nanofluid.

The second portion of the chapter will discuss the experimental model and how the model reflects the generalized theory of the experimental method used to measure the thermal conductivity. The material, the positioning of the fluids, and the temperature distribution within the model will be examined. In addition, the experimental procedure will be briefly discussed.

\subsection{Visibility of fringes}

Prior to the current project, a preliminary issue that required investigation was the visibility of interference fringes in nanofluids. The concern was due to the size of the experimental model and the transparency of the nanofluid. If the volume concentration of nanofluids was increased, the transparency of the fluid would be reduced correspondingly. For that reason, a simple experiment was performed by Yousefi et al. (2015), where visible fringes were achieved for a laser path length of $0.7 \mathrm{~cm}$, which is close to the path length used for the current experiment $(1 \mathrm{~cm})$. Figure 2.1 and Figure 2.2 demonstrate the quality of the temperature profiles produced from the interferometer for both $\mathrm{Al}_{2} \mathrm{O}_{3}$ and $\mathrm{SiO}_{2}$ at nanofluid concentrations of $0.1 \%$ and $0.07 \%$ by weight respectively. It can be seen that a clear interference fringe pattern was achievable up to the concentration of interest in the current experiment, which will be up to a volume concentration of $20 \%$ with $\mathrm{SiO}_{2}$-water nanofluid. 


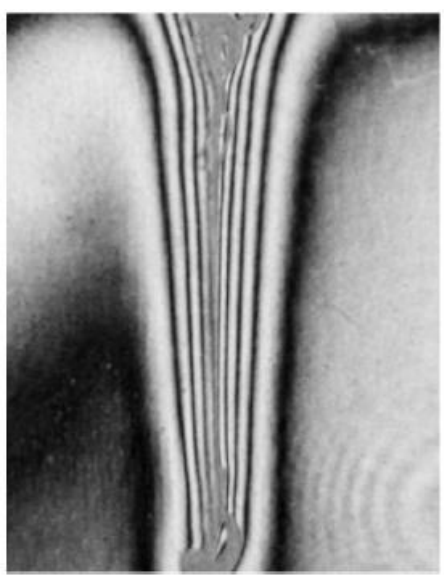

Figure 2.1: Infinite fringe interferogram for $\mathrm{Al}_{2} \mathrm{O}_{3}$-water nanofluid; $0.1 \%$ wt after homogenization (Yousefi et al., 2015).

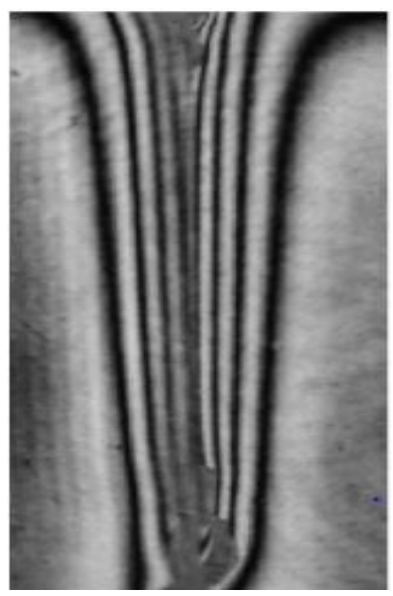

Figure 2.2: Infinite fringe interferogram for $\mathrm{SiO}_{2}$ - water nanofluid; $0.07 \%$ wt. (Yousefi et al., 2015).

The quality of the interference fringes was examined for two nanofluids at varying concentrations in Yousefi's experiments. For the $\mathrm{SiO}_{2}$-water nanofluids, weight concentrations from $0.07 \%$ to $40 \%$ were shown to yield visible fringes in the interferometer. As for $\mathrm{Al}_{2} \mathrm{O}_{3}$-water nanofluid, clear high-quality fringes were captured up to $1 \%$ by weight concentration. It was important to mention, performing experiment with a complete homogenous solution played an important role to achieve visible fringes. The process of transforming the heterogenous nanofluid solution into a homogenous solution was to sonify the mixture for 4-8 hours. The sonification process prevents sedimentation of the nanoparticles to be present in the nanofluid solution. In addition, many existing studies on measuring the thermal conductivity of nanofluids particularly for $\mathrm{Al}_{2} \mathrm{O}_{3}$ and 
$\mathrm{SiO}_{2}$ have experimented with volume concentrations ranging from $0.05 \%$ to $1 \%$. However, due to the measurement uncertainty of the model's ability to effectively measure the thermal conductivity of water, the volume concentration above $7 \%$ with $\mathrm{SiO}_{2}$-water nanofluid will be investigated in this research.

Note that the images seen in Figure 2.1 and 2.2 were set with the infinite fringe mode. There are two available settings to be used when a picture is captured from the interferometer. These are the infinite and finite fringe settings. Traditionally, infinite setting will be used for a visual display of the temperature field. In this mode, fringes are isotherms. The finite setting mode will be used when making measurements from temperature field. The output from the interferometer will display a constant fringe gradient parallel to the divider plate that is superimposed upon the fringe field due to the temperature field. Figure 2.3 is an example of a captured image from the experiment performed using the proposed technique. The captured image demonstrates visible fringes in the nanofluid domain. A high-quality interference pattern in both fluid domains was achieved with the model developed in this project.

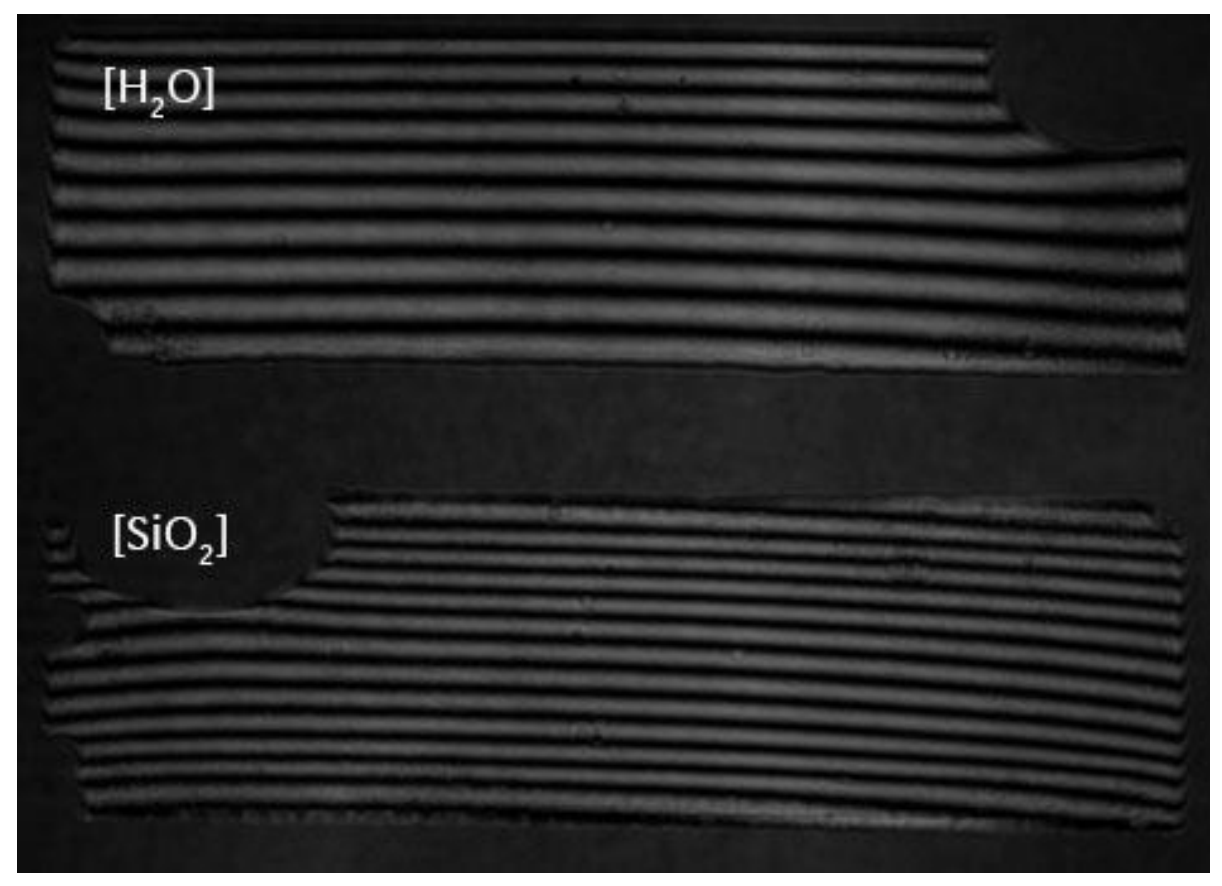

Figure 2.3: Experiment performed with $\mathrm{SiO}_{2}$-water nanofluid at $10.1 \%$ by volume concentration using finite fringe setting. 
Another critical factor that can be taken from Yousefi et al. experiment was the path length used to achieve visible interference pattern. The path length used in Yousefi's experiment was $0.7 \mathrm{~cm}$. which has validated that the path length used in the current model for this project will be successful. The path length used in the model was set to $1 \mathrm{~cm}$, which was close to the path length used in Yousefi's experiment. The path length was one of the required objectives in the development of the experimental technique to achieve visible fringes.

As mentioned in a previous paragraph, the procedure used to mix the nanofluids is a significant factor in achieving clearly visible fringes. Aside from the requirement to use a transparent fluid, the homogeneity of the nanofluid is important. If the nanofluid is not mixed thoroughly, the resulting particles will not be uniformly distributed, and the experiment would result in a poorquality data. Firstly, the particles will not be dispersed, and secondly the nanoparticles will be settling on the surface. As a result, the documented concentration does not correctly reflect the locally measured concentration. Thus, adequate mixing the commercial nanofluid was a critical part of the experimental procedure. A process that has been used to mix the nanofluid to evenly distribute the nanofluid was done with an ultra-sonification technique. A probe was submerged into a solution and emits sound waves to agitate the particles in the solution. The system converts the electrical signals into physical vibrations, breaking down the solid particles to uniformly distribute the particles in the solution. Experiments with nanofluids have shown that this process of mixing the nanofluid was optimal for research purposes. Transmission electron microscopy can analyze the nanoparticles characterization and have demonstrated the nanoparticles in the base fluid are uniformly distributed in a nanofluid after undergoing a sonification for more than 8 hours. After this process, the nanofluid can maintain its properties for a long period in a cold environment. Thus, a sonification process will be done prior to the experimental measurements to ensure the nanoparticles are evenly distributed in the solution.

As mentioned in Chapter 1, there is an important variable that has been neglected in previous studies involving low volume concentrations of the nanofluids. Many of the studies that predicted the thermal conductivity have miscalculated the measurement uncertainty at low volume concentrations. That particular variable is the temperature coefficient of refractive index, which is needed to calculate the temperature gradient from the fringe gradient. Researchers that have investigated the nanofluids (Haridas et al., 2015; Rajput et al., 2016) at volume concentration 
below $0.05 \%$ have assumed the temperature coefficient of refractive index to be equal to that of pure water $(\phi=0 \%)$. However, recently Yousefi et al. [2019] performed an experiment to investigate the temperature coefficient of refractive index at a function of both weight percent $(0$ $40 \%$ ) and temperature as shown in Figure 2.4. The data shows significant changes in the temperature coefficient of refractive index as the weight concentration increases. The result demonstrates the temperature coefficient of refractive index could influence the measurements that are used in optical techniques to measure the thermal conductivity of $\mathrm{SiO}_{2}$-water nanofluid.

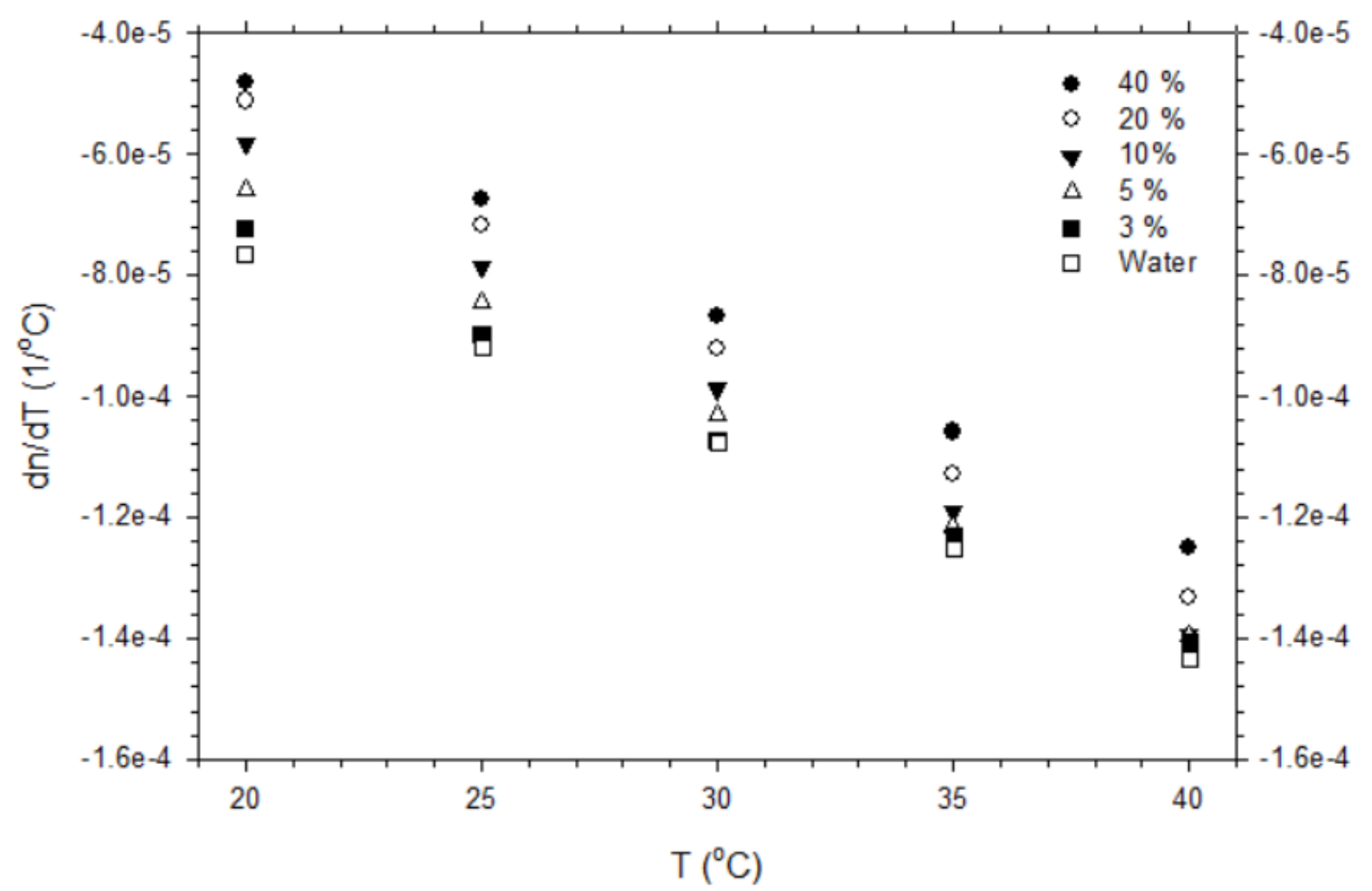

Figure 2.4: Temperature coefficient of refractive index at varying temperatures and weight concentrations with $\mathrm{SiO}_{2}$-water nanofluid (Yousefi, 2019). 


\subsection{Introduction to interferometry}

Interferometry is a non-intrusive measuring technique that can provide a real time measurement of the refractive index, from which the temperature field can be obtained. There are various optical techniques that are used for heat transfer research, and the interferometry setup that will be used for the current project is the Mach-Zehnder interferometer. This optical technique provides a visual display of the temperature field in the experimental model, allowing for measurements to be made and heat transfer properties to be investigated.

There are a few advantages of using the Mach-Zehnder interferometry. A key feature is that the technique is non-intrusive to either temperature field or the flow field, which results in measurements with minimum disturbance. In particular with the current setup, the large displacement of the test beam compared to the reference beam provides the opportunity to improve the clarity of the processed images and the accuracy of the alignment. As a result, the technique to measure the temperature gradient in real-time becomes more reliable.

On the other hand, there are a few drawbacks of using the Mach-Zehnder interferometry. The measurements are best suited for two-dimensional temperature fields, and experiments under laminar flow conditions. The fluid that will be experimented is required to be transparent to laser light, in order for a visible display to occur in the interferometer. The need for transparency limits the type of nanofluids that can be tested. Due to the sensitivity of the entire procedure, the environment of the room is required to be clean and the level of vibration must remain as low as possible. It is important to note that the interferometry consist of expensive and fragile components, which will require a skilled operate and careful handling to occur during the entire procedure.

\subsubsection{The Mach-Zehnder interferometer}

In 1995, Von Bistram [1995] built the Mach-Zehnder Interferometry at Ryerson University that was based on Tarasuk's design (Tarasuk, 1968) and the interferometry at the University of Western Ontario. The properties of the optical component that are used in the interferometer are available in appendix B.1. More information on the design and optical specifications can be found in Roeleveld's doctoral thesis (Roeleveld, 2013). 
A schematic diagram of the Mach-Zehnder interferometry setup used in the current experiment is shown in Figure 2.5. The laser used in the interferometer is a $15 \mathrm{~mW}$ Helium-Neon laser, producing a wavelength of $\lambda=632.8 \mathrm{~nm}$. The interferometer consists of two beam splitters, a beam expander, two flat mirrors, a parabolic mirror, and a spherical mirror. An additional component that will be used in the procedure will be a spatial filter for filtering the light. Also, a small mirror will be used to reflect the light towards the camera. The process of interferometry starts off with the laser directed towards a spatial filter that will remove some of the high frequency noise in the light beam before encountering the beam expander. The beam expander will enlarge the collimated beam to a diameter of $20 \mathrm{~cm}$, which is then directed towards a parabolic mirror. The beam will then travel to the first beam splitter (BS1) to create two identical collimated beams (reference beam and test beam) both heading towards a flat mirror. The test beam will be passing through the experimental model, which will result in the speed of light of the beam changing due to the fluid in the experimental model. In contrast, the reference beam passes through the same medium from the start, which is air. Thus, the change in the speed of light will result in a phase difference when both beams are reconstructed at the second beam splitter (BS2). The phase shift that exists at BS2 results in the formation of constructive and destructive fringes when projected onto the spherical mirror and camera. Right after the beam has reflected off the spherical mirror, the light will target a small mirror that will reflect the light towards the camera.

All the optical components in the setup have specific features to achieve interference patterns and minimize the measurement uncertainty performed with the interferometer. Important features of the optical components are the anti-reflective coatings on the beam splitters, surface flatness of the mirrors $(\lambda / 20)$, and the optical alignment.

The beam splitters have different specifications on each side. The front side is 50\% reflective and $50 \%$ transmissive, and the back side of the splitter is coated with an anti-reflective layer. The positioning of the beam splitter is an important step to achieve the best quality in the captured image.

The surface flatness and parallelism of the beam splitters is an important feature when designing the interferometer. If the optical surfaces are not parallel, the output of the interferometer will have a wave front error. In that scenario, the light will be out of phase, because a portion of the light will pass through a thinner part of the optic, and a portion through a thicker portion of the optic, 
resulting in a wave front distortion. The beam splitters that are used in the interferometry will be flat to $1 / 20$ of a wavelength of light.

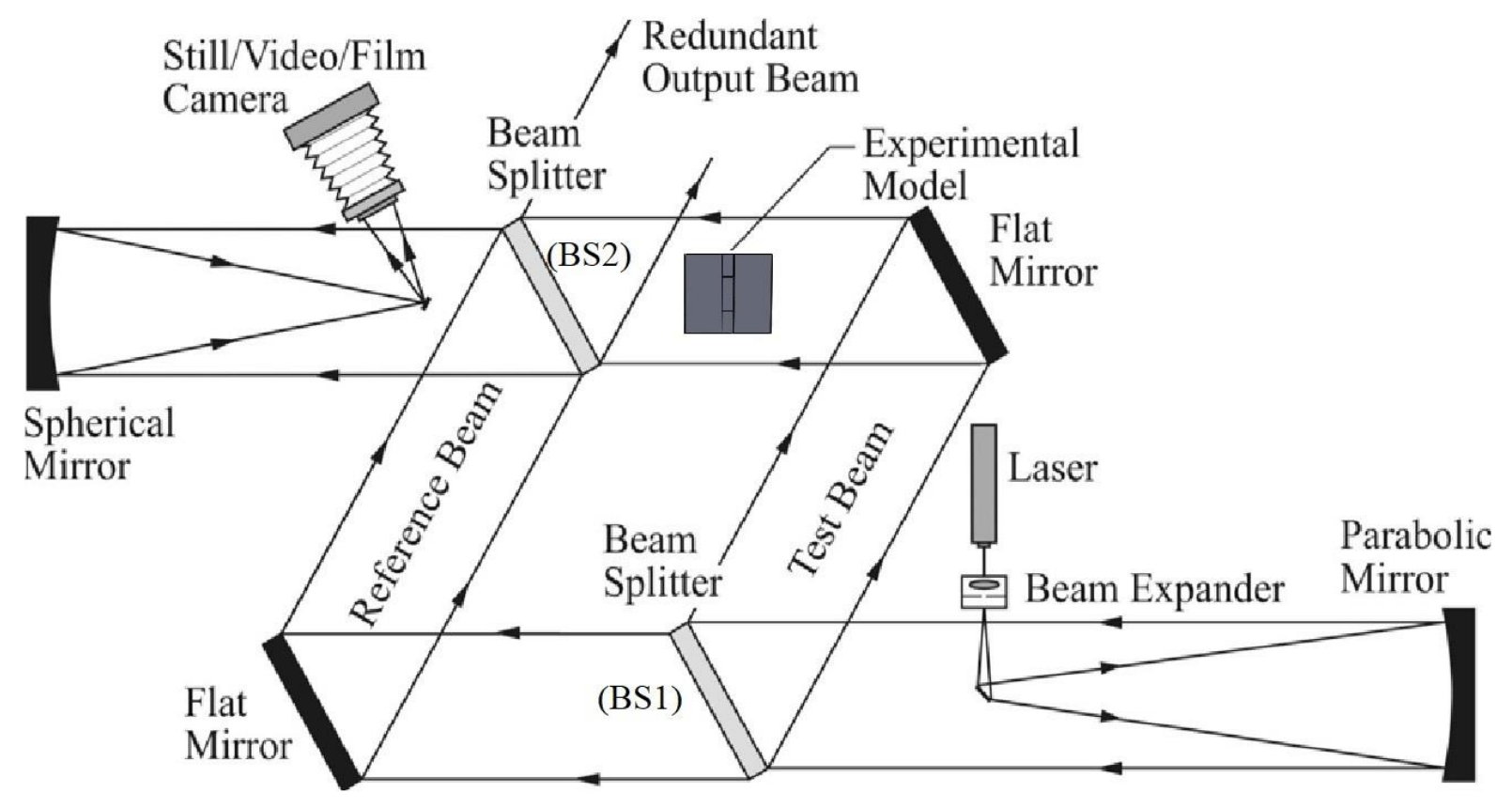

Figure 2.5: Setup of the Mach-Zehnder Interferometer.

The table that each individual optical component will be mounted on was manufactured by Newport Corporation. The optical bench weighs $800 \mathrm{lbs}(364 \mathrm{~kg})$ and has dimensions of $1.22 \mathrm{~m} \times$ $3.05 \mathrm{~m}$ (4 feet $\times 10$ feet) with 1/4 - $20 \mathrm{NC}$ tapped holes equally spaced on $25.4 \mathrm{~mm}$ ( 1 inch) center. The external vibrations from the floor were reduced by placing 8 inflated tire inner tubes under the table. The laser, first beam splitter (BS1), flat mirror for the test beam and parabolic mirror were mounted on the table. The second beam splitter (BS2) and flat mirror for the test beam were mounted on an extension that was cantilevered off the edge of the optical bench (Roeleveld, 2013). 


\subsection{Interference fringe analysis}

The analysis of the interferogram is based on the wave nature of light. The amplitude of the monochromatic light emitted from the laser in the $\mathrm{z}$ direction towards the experiment can be expressed as the following:

$$
a=a_{o} \sin \frac{2 \pi}{\lambda}(c t-z)
$$

Where $a_{o}$ the maximum amplitude, $\mathrm{c}$ the speed of light, $t$ the time, $\mathrm{z}$ the position, and $\lambda$ the wavelength. From that information, the amplitude of the reference beam at a fixed location can be expressed as the following:

$$
a_{r e f}=a_{o, r e f} \sin \frac{2 \pi c t}{\lambda}
$$

Similarly, the amplitude of the test beam at a fixed location can be expressed as:

$$
a_{\text {test }}=a_{o, t e s t} \sin \left(\frac{2 \pi c t}{\lambda}-\phi\right)
$$

where $\phi$ is the phase shift between the two light beams, because of the changes in the refractive index of the fluid in the experimental model.

Assuming that both waves have identical amplitudes $\left(a_{o}=a_{o, r e f}=a_{o, t e s t}\right)$, then the recombined equations of both beams merging at the second beam splitter would be expressed as:

$$
a=a_{o}\left(\sin \frac{2 \pi c t}{\lambda}+\sin \left(\frac{2 \pi c t}{\lambda}-\phi\right)\right)
$$

The interferometry measures the fringes shift of the two waves, which is caused by the change in the refractive index in the test section. A fringe shift $(\varepsilon)$ is a difference in the number of wavelengths in the reference and test beams. The expression of the fringe shift at the surface of the thin barrier is as follows:

$$
\varepsilon=N-N_{\text {ref }}
$$

where $\mathrm{N}$ is the number of wavelengths, which is determined by the path length of the experimental model divided by wavelength, and is expressed as the follows:

$$
N=\frac{L}{\lambda}
$$


The index of refraction (n) is the ratio of the speed of light in a vacuum $\left(\mathrm{c}_{\mathrm{o}}\right)$ to the speed of light in the medium (c), which is expressed as:

$$
n=\frac{c_{o}}{c}=\frac{\lambda_{o}}{\lambda}=\frac{N_{o}}{N}
$$

Over an optical path length L, the fringe shift is equal to the difference in the number of waves in the test beam minus the number of waves in the reference beam. Hence, the fringe shift equation can be written as:

$$
\varepsilon=L\left(\frac{1}{\lambda}-\frac{1}{\lambda_{\text {ref }}}\right)=\frac{L}{\lambda_{o}}\left(n-n_{\text {ref }}\right)
$$

Differentiating equation 2.8 with respect to $\mathrm{x}$ gives the equation for the fringe gradient:

$$
\frac{d \varepsilon}{d x}=\frac{L}{\lambda} \frac{d n}{d x}
$$

Refractive index gradient $(\mathrm{dn} / \mathrm{dx})$ can be expressed in terms of the temperature gradient and the temperature coefficient of refractive index by using the chain rule equation, shown in equation 2.10:

$$
\frac{d n}{d x}=\frac{d n}{d T} \frac{d T}{d x}
$$

where $\mathrm{dn} / \mathrm{dT}$ is the temperature coefficient of refractive index. The data for $\mathrm{dn} / \mathrm{dT}$ for the nanofluids used in the current experiment will be based on the experimental results of Yousefi et al. (2015)

By expanding the fringe gradient in equation (2.9) by using the chain rule on the refractive index gradient will be the equation to solve for the fringe gradient.

$$
\frac{d \varepsilon}{d x}=\frac{L}{\lambda_{o}} \frac{d n}{d T} \frac{d T}{d x}
$$

where $d \varepsilon / d x$ is the measured fringe gradient from the interferometer, and $d T / d x$ is the temperature gradient. Re-arranging the equation and solving for the temperature gradient at the surface of the thin barrier gives: 


$$
\left.\frac{d T}{d x}\right|_{S}=\frac{\left.\frac{d \varepsilon}{d x}\right|_{S}}{\frac{d n}{d T}} \frac{\lambda_{o}}{L}
$$

\subsubsection{MATLAB image processing code}

MATLAB was used to process the digital interferograms to obtain the fringe gradient at the surface of the fluid barrier. An overview of the MATLAB code was discussed to understand the steps performed within the computation process. The image captured from the interferometry will require a mathematical analysis to determine the required information to solve the heat transfer problem. The code itself has a unique procedure to reduce the effects of optical imperfections on the processed image to improve the precision of the data.

A breakdown of the process used in the MATLAB code will be explained in a procedural manner starting from the moment the images were processed to obtain the fringe spacing of the experimental model obtained from the interferometer. A Phase One P45 digital back camera with a resolution of 39 mega pixels was used to capture the still image. The captured image file will be a grey scaled image with 8-bit digitization. An 8-bit digitization indicates the number of bits in a binary representation identifying the brightness level. There are 256 shades of gray in a grayscale image. Before the regression analysis was performed, a median filter was active to modify the captured image. A median filter is a nonlinear digital filtering analysis to reduce the imperfections such as noise or other content reflected from the lens. The size of the median filter was determined by a kernel method. The kernel method determines the size of the filter based on the resolution i.e., the number of peak to peak pixels. The size of the kernel will always be an odd number. Once the median filter has improved the image file, a non-linear regression analysis which includes the equations 2.1-2.4 to generate an intensity variation normal to the surface that is approximated by a sine curve. The regression algorithm was based on Slepicka's (1995) research on the study of interferogram analysis. The curve that was generated is a least square best fit to a single wave based on the spatial coordinates. If the best fit curve is a close fit to the fringe data, the information to calculate the fringe gradient is outputted by the program. From this step on, the fringe gradient was used with the measurement technique to measure the relative thermal conductivity. 


\subsection{Experimental model}

A general summary of the model's design will be discussed to provide a breakdown of the important features that were required to achieve the objective for this project. Figure 2.6 shows an isometric view of the complete model that was used to experimentally measure the thermal conductivity of $\mathrm{SiO}_{2}$-water nanofluid. As seen in Figure 2.6, the shape of each individual part of the design will be rectangular with slight modification to the shape such as grooves for O-ring, and screw holes. There will be two fluid domains of the same dimension, and a thin aluminum solid barrier placed in between the fluid domains. The thin barrier is designed to ensure the heat flux through the barrier is the same. By selecting the thin barrier to be aluminum with a thermal conductivity of hundred times greater than those of the fluids being used, the temperature gradient along the direction of the heat transfer will be as close to zero. A thin high conductivity barrier is used so that the heat flux at the surface of the upper fluid domain is the same as the heat flux at the surface of the fluid on the bottom of the thin barrier. By understanding the structure of model, the next step will be looking into each of the component that will be required and its contribution to the design. As well, each of the individual components that make up the complete model shown in Figure 2.6 is available in Appendix C, with the specific dimensions and materials.

In order to perform measurements at the required temperature with roughly the same temperature difference on both sides of the design, specific components will be required. Aside from the thin aluminum barrier at the center of both fluid domains, hot and cold sources are required for the design to achieve the targeted temperature. Thus, a hot surface at the top of the water domain and a cold surface at the bottom of the nanofluid was set. The desired temperature for both surfaces will be controlled by a bath, supplying water at the targeted temperature. The Corsair Liquid CPU cooler component was used to heat/cool the fluid domain by the flow of water supplied by the water bath. To ensure a stable temperature distribution will exist in the experiment, the surface will be in contact with an aluminum block was heated/cooled to the desired temperature. After 20 minutes have passed the experiment is expected to have reached steady state. The three thermocouples were checked to ensure the desired temperatures were met and steady. Once achieved, the experiment could begin. For the current experiment, the targeted temperature was $20^{\circ} \mathrm{C}$ with a temperature difference of $10^{\circ} \mathrm{C}$. The laser was passed through the experimental model (in the z-direction). The surface on the front and back side of the model needs to contain a 
transparent surface. Thus, two transparent glass windows that are flat to 1/10th of the wavelength and that have a thickness of $4.5 \mathrm{~mm}$ was used.

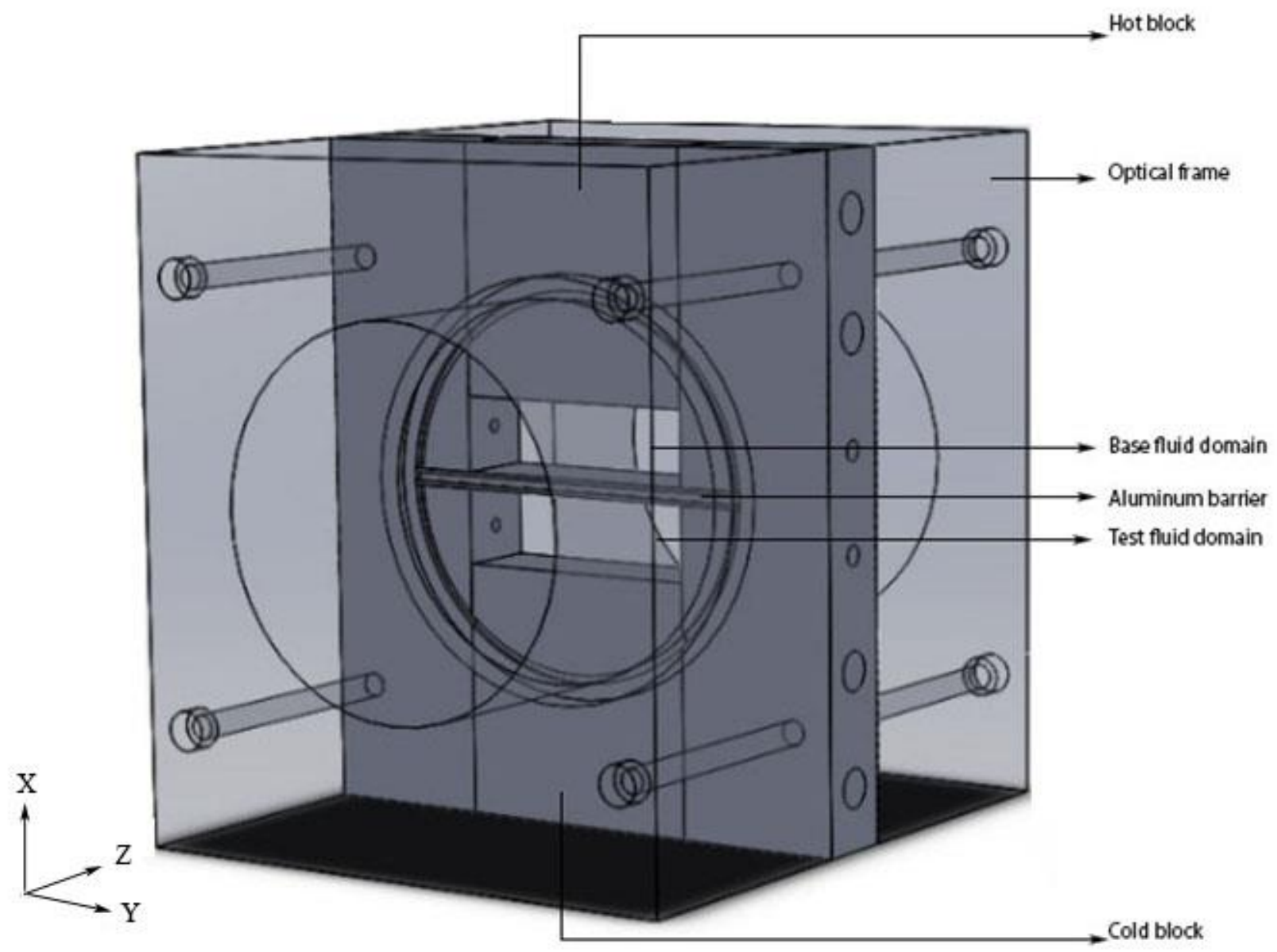

Figure 2.6: Isometric view of the experimental model.

Furthermore, the fluid domains require attention in the construction of model design. The fluids were arranged to have the water placed to be above the nanofluid, due to the difference in the density. The density of the nanofluids is expected to be greater than of water because of the nanoparticles. If leakage became an issue during the experiment, the water will remain on top of the nanofluid, because the denser fluid will maintain its position in lower fluid domain. Information on how leakage was prevented in the design of the model will be discussed in Chapter 5. 


\subsection{Thermal conductivity measurement}

At this point, the concept of the design and the governing equations associated with the interferometry have been explained in order to understand the experimental method used to measure the thermal conductivity. As mentioned earlier, Fourier's equation is the foundation for the measurement of the thermal conductivity of the nanofluid. The heat transfer rate is measured at the surface of the barrier. At the surface the heat transfer occurs by pure conduction due to the no slip condition. As for the thin barrier separating the two fluids in the experimental model, the heat flux at the surface of the thin barrier on the top side should be the same as the heat flux at surface of the bottom side, which is demonstrated in equation (2.13). When the equation holds true during experiment, the Fourier's equation for both fluids can be equated to each other which form the fundamental equation for the experimental technique and demonstrated in equation (2.14).

$$
\begin{gathered}
q_{t o p_{s}}=q_{\text {bottom }_{s}} \\
\left.k_{b f} \frac{\partial T}{\partial x}\right|_{s}=\left.k_{t f} \frac{\partial T}{\partial x}\right|_{s}
\end{gathered}
$$

where $\mathrm{k}_{\mathrm{bf}}$ is the conductivity of the base fluid and $\mathrm{k}_{\mathrm{tf}}$ is the conductivity of the test fluid.

Solving for the thermal conductivity of the nanofluid by re-arranging equation (2.14):

$$
k_{n f}=\frac{\left.k_{w} \frac{\partial T}{\partial x}\right|_{s}}{\left.\frac{\partial T}{\partial x}\right|_{s}}=\frac{k_{w}\left(\left.\frac{d \varepsilon}{d x}\right|_{w}\right)\left(\frac{d n}{d T_{n f}}\right)}{\left(\left.\frac{d \varepsilon}{d x}\right|_{n f}\right)\left(\frac{d n}{d T_{w}}\right)}
$$

To determine the temperature gradient at the surface of the thin barrier in both fluid domains was achieved by using equation (2.15). Equation (2.15) is dependent on the fringe gradient from both fluid domains that was extracted from the interferometry using the MATLAB code. The thermal conductivity of the water $\left(\mathrm{k}_{\mathrm{w}}\right)$ at $20^{\circ} \mathrm{C}$ will be taken from Touloukian's data (1970). The data for water was estimated to have an uncertainty of less than one percent. This data comes from several experimental techniques performed decades ago.

The accuracy of the technique to measure the thermal conductivity experimentally will be assessed by making measurements with water as the test fluid. Note from equation (2.15) that one of the advantages of this comparative method is that the uncertainty in the temperature difference 
measurement (from the thermocouples) does not directly influence the accuracy of the thermal conductivity measurement. Furthermore, unlike most other studies involving the interferometer, the uncertainty scale factor of the captured image does not influence the measurement. This is because the scale factor is the same in both fluid domains. 


\section{Chapter 3: Experimental Apparatus and Procedure}

\subsection{Introduction}

In this chapter, a summary of the apparatus, including the model, frame, and the associated equipment will be explained, as well as the procedure used to execute the experiments. The first portion of the chapter will provide a breakdown of the model, and the method to assemble the model, as it is a necessary process when working with new fluids (i.e., water and nanofluids). Additionally, the customized frame that was designed to provide the model with stability during the experimental test will be explained. Also, each of the essential equipment that is used to perform experiments in the interferometer will be discussed in this section. The second portion of the chapter describes the step by step procedure to perform the experiment and extract the information needed to calculate the relative thermal conductivity of the test fluid.

\subsection{Experimental apparatus}

In developing an experimental apparatus for this project, a list of components was prepared for the experiment to perform smoothly. In addition to the components involved in construction of the experimental model, a set of equipment was needed for a specific task to be met. The collection of equipment used for the experiments is categorized as follows:

- Interferometer equipment

- Calibration and leveling tools

- Temperature control units

- Tools for fluid management

A schematic of the model and the major components of the experiment are shown in Figure 3.1. The figure illustrates the essential components that were used in the experiment. The water baths supplied a flow of water at a constant temperature to the liquid CPU cooler units, which was used to heat the model to the desired temperature. The location of the three thermocouples is indicated. The support frame was designed to control the model's stability. The support frame was attached to a height-adjustable table, which allowed the model's position to be adjusted in the interferometer's test beam. 


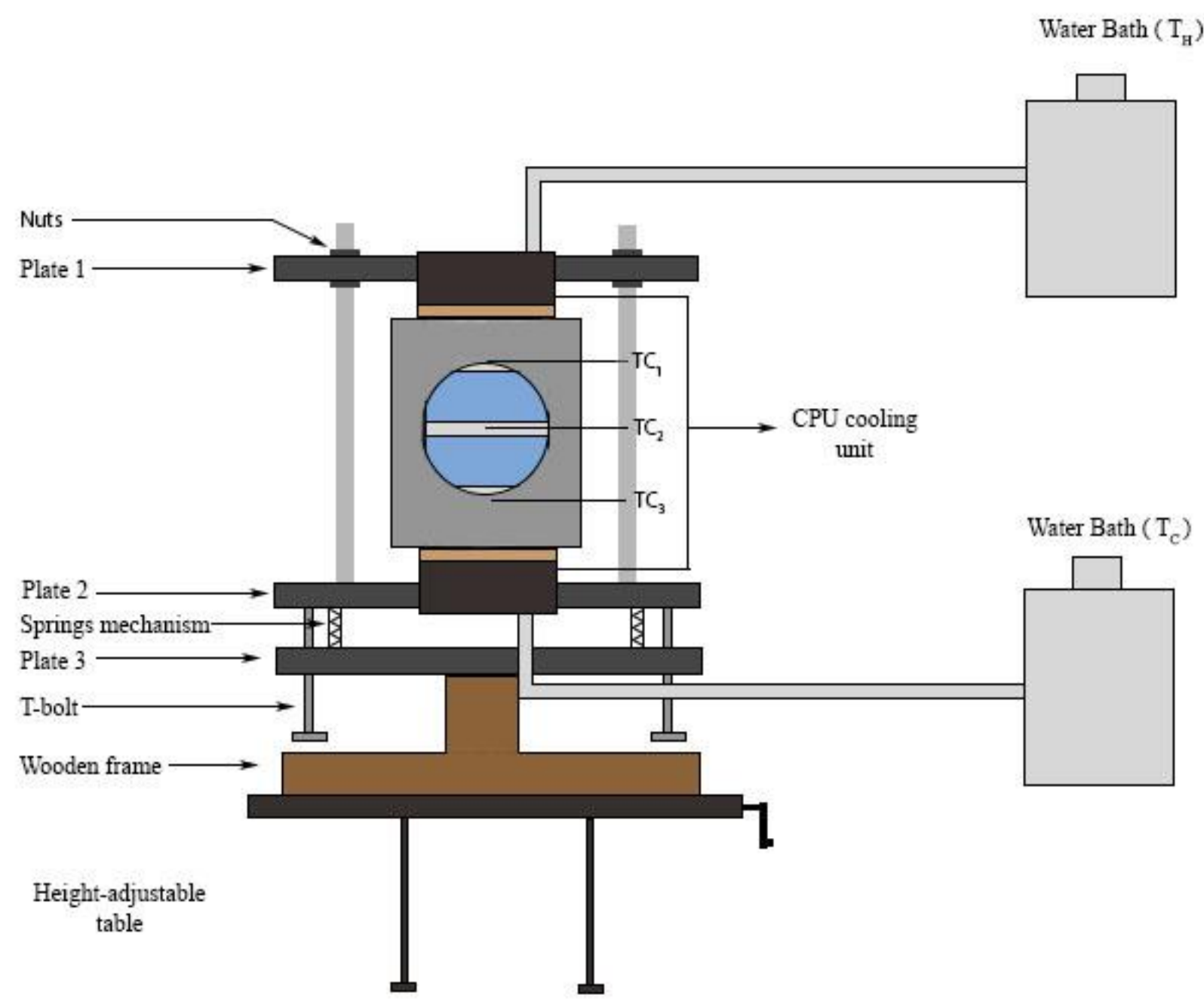

Figure 3.1: Apparatus of the experiment.

\subsubsection{Interferometer equipment}

\section{Optical components}

As discussed in Chapter 2, there is a list of parts which are needed to operate with the interferometer. Figure 2.5 shows the setup of the Mach-Zehnder interferometer (MZI) and the locations of each optical component. Prior to each experiment the optical components of the MZI were carefully aligned using the near-field/far-field procedure of Tarasuk [1968]. 


\begin{abstract}
Airbags
The interferometer is located on the third floor of Eric Palin Hall. In order to obtain stable interference patterns for analysis, the MZI must be isolated from building vibrations. For this reason, all of the optical components of the MZI are mounted on an optical table, which sits on eight air bags. The air bags greatly attenuate the building vibrations and allow optical table to be leveled.
\end{abstract}

\title{
3.2.2 Calibration and leveling tools
}

\section{Digital level}

Performing measurements on a leveled platform is a crucial element for this technique. The main concern is that the heated and cooled surfaces in the test model are horizontal, in order to minimize free convection in both the test and base fluids. Prior to each experiment, the experimental model set in the horizontal position $\left( \pm 0.5^{\circ}\right)$ with a digital level. In addition, the interferometer test beam was adjusted to pass horizontally through the model.

\section{Digital scale}

The process of preparing a lower volume concentration nanofluid than the commercially prepared nanofluid will require a digital scale. The calibrated digital scale was required to measure the weight of the deionized water used to dilute the commercial nanofluid to achieve the desired nanofluid at a lower concentration. The digital scale with an accuracy of \pm 0.05 grams was used to prepare the nanofluids for the experiments.

\section{LED/flashlight}

A small LED light source was used to focus the camera on the center plane of the test model. Focusing the camera enhances the quality of the image and reduces the measurement error caused by beam refraction.

\section{Glass thermometer}

A calibrated glass thermometer was required to calibrate the thermocouples used in the model. The glass thermometer was calibrated by the manufacturer, which was purchased from H-B 
instruments. The measurement resolution was $0.1 \mathrm{~K}$. The calibration of the thermocouples was performed with the glass thermometer using the constant temperature bath. The data is available in Appendix G.1.

\section{Supporting frame for the model}

It was necessary to construct a customized frame to secure the model in position and to maintain the model's stability during the experiment. The design of the structure had two purposes in terms of minimizing the potential errors in the experimental results. The first goal was to position and secure the model in place, along with the equipment associated with the CPU cooling system. The second goal was to ensure that the model was resting on a leveled platform, which was achieved by using a spring mechanism underneath the plate supporting the model. The springs were implemented on three corners of the platform to level the surface supporting the model. Figure 3.2 shows the components of the frame, including the bolts and fasteners. The plate numbers labeled in Figure 3.2 will be referred to in the procedure. Since the frame was a simple concept, the entire frame was made from aluminum and was mounted onto a wooden base. Figure 3.3 shows the frame

firmly locked onto the table in the position needed to secure the model in the test beam for the experiment. 


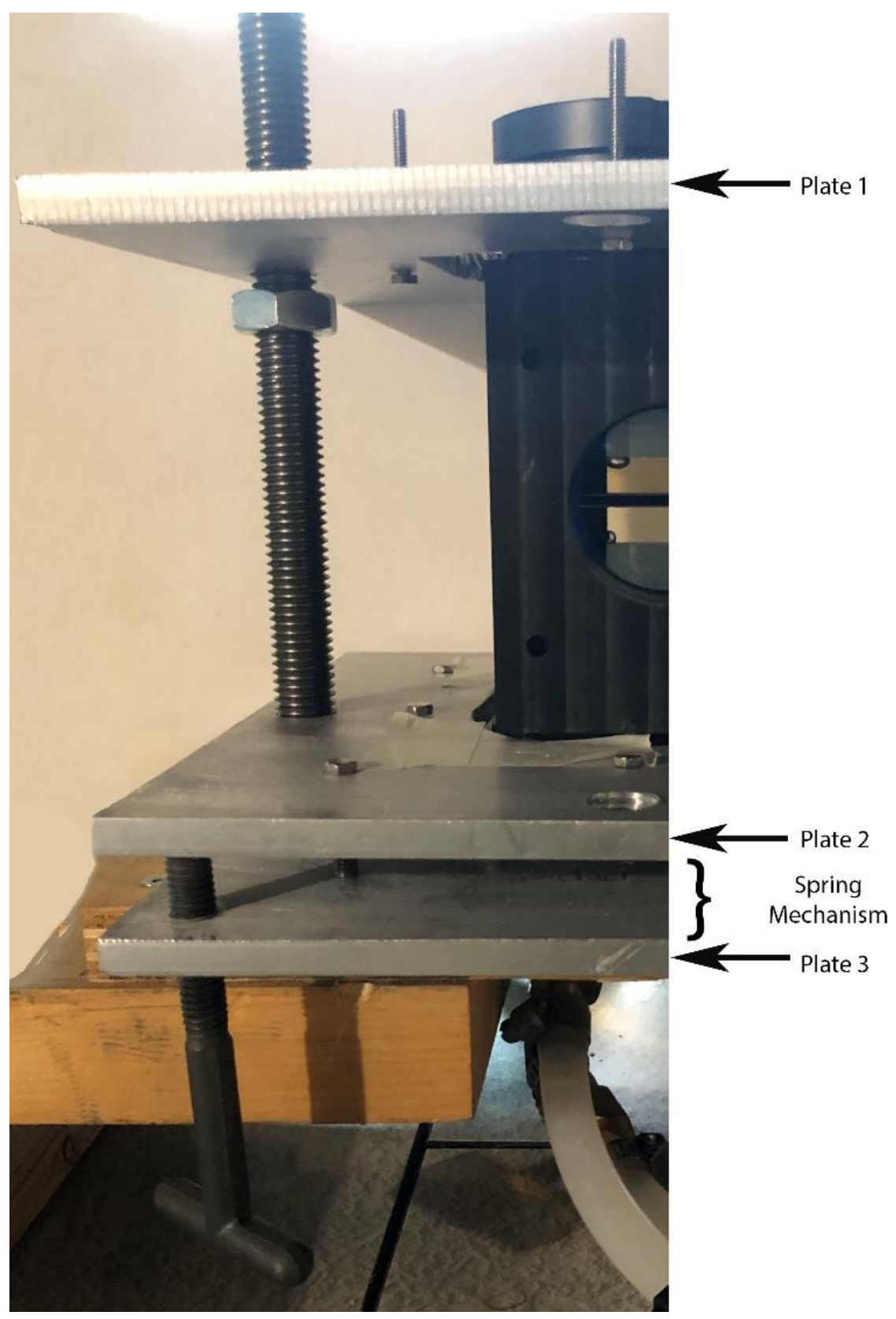

Figure 3.2: Frame design with the model tightened in position. 


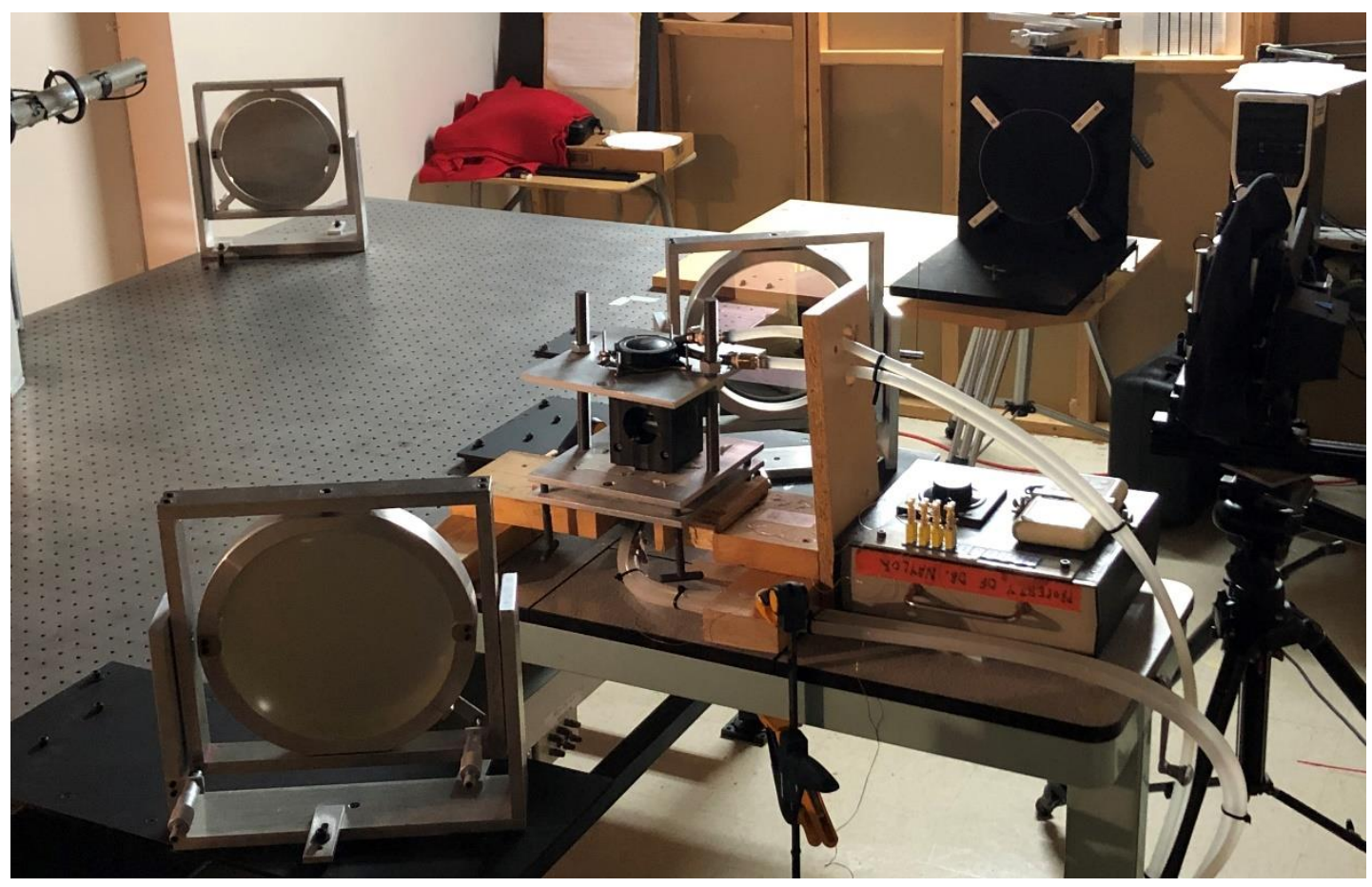

Figure 3.3: Full view of the frame within the interferometry setup.

\subsubsection{Temperature control units}

\section{Liquid CPU cooler}

The model was heated and cooled using two liquid CPU cooling units. Each CPU cooling unit was heated/cooled by a flow of water from a temperature-controlled water bath. The CPU cooling unit is a simple heat exchanger in contact with the copper plate as shown in Figure 3.4. The temperature of the copper plate will be approximately the same temperature as the water bath. Simultaneously, the temperature of the hot and cold blocks in the experimental model will be about the same temperature as the copper plates of the CPU cooling units. A slight modification was made to the two commercially available liquid CPU cooler units. To allow a free flow of water through the heat exchangers, the pump components within the CPU cooler units were removed. 


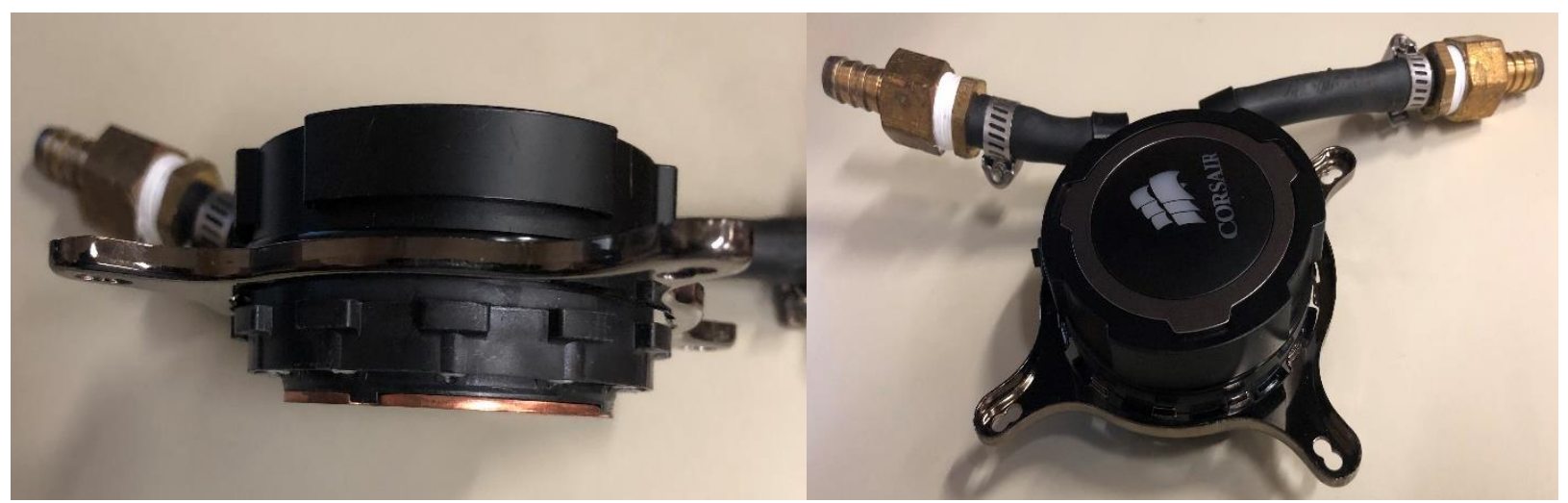

Figure 3.4: CPU cooling units with modifications to connect to the water bath (front and top view)

\section{Water baths}

The measurement technique requires a desired temperature gradient to be maintained within the model. Two LAUDA PROLINE RP 1845 refrigerated circulating water baths were used for this research. These baths pump water to the model's CPU cooling units and have PID temperature control.

\section{Thermocouples}

The thermocouples used for the project were Type $\mathrm{T}$ and a 30-gauge size. The locations of the thermocouple are highlighted in figure 3.1. The thermocouple in the barrier was used to maintain the barrier at a temperature of $20^{\circ} \mathrm{C}$, in both unheated and heated states. The other two thermocouples were used to ensure that temperature gradient in the bottom fluid domain was the same as in the top fluid domain.

\subsubsection{Tools for fluid management}

\section{Fluid injection technique}

The process of injecting the fluid is complicated due to the design of the model. The sizing of the holes for the fluid to enter and leave the fluid domain are extremely small. A $1.3 \mathrm{ml}$ fine stem pipette was used with an extended tip diameter of $2 \mathrm{~mm}$. The pipette was filled with the experimented fluid roughly four times to completely fill the fluid domain with the air flushed out 
within the space. To prevent contamination, two pipette were used for each fluid placed in the two fluid domains. The pipette filled with the fluid was inserted into the fluid domain inlet hole, and once the pipette tip was inserted in the fluid domain, the fluid was injected into the domain.

\section{Fluid storage}

The nanofluid solution used in the experiment must be kept in a secure container for several reasons. Both the deionized water and the nanofluid used in the experiment needs to be free from contamination. In the process of sonifying the nanofluid, the typical procedure requires the solution to be poured into a separate container to mix the solution and be poured back into the sample container, increasing the chances of the solution to be contaminated. The size of the container used in the project was enough to insert the homogenizer's probe and mix the fluid in the same container used to store the fluid. The potential health risk associated with the nanofluid is unknown, and for the safety of individual's health and environment, the nanofluid must be kept in a secure container to prevent spillage.

\subsubsection{Model}

The model consists of ten major components, including the optical windows. The drawing of each component of the model is shown in Appendix C, along with a list of the materials that were used. There were two primary materials used to design the model, excluding the fasteners, which were aluminum and Delrin that were purchased from McMaster-Carr. The thin barrier dividing the fluids, and the hot/cold blocks located on the top and bottom of the model, are made of aluminum. The upper hot block and lower cold block were maintained at the desired temperatures by a flow of water to two CPU cooling units, which were connected to constant temperature baths. The optical frame (designed to maintain the optical window in a fixed position) and the side blocks (designed to secure the hot/cold block) were made of Delrin. Figure 3.4 shows a photo of half of the model that was precisely assembled. The precision of this partially assembled model was used as the reference for the $\mathrm{CNC}$ machining process to machine the remaining parts of the model. The difficulty in manufacturing the model was due to the required tolerances of the individual components and the assembly the model as one piece without any misalignments. Thus, the assembly shown in Figure 3.5 should not be dismantled due to the possibility of interrupting the alignment of the model. 
Figure 3.6 shows the Delrin component responsible for holding the optical window (identified as optical frame). Once the optical window was set in position, and the O-ring applied with silicon lubricant and placed in the O-ring groove, the components were carefully aligned and assembled. Threaded nuts, fitted to the fastener, were used to hold the model together. The threaded nuts were carefully tightened, as over tightening the assembly could bend the optical windows. (The implications of bending of the optical windows are discussed in Chapter 5.) Figure 3.7 shows the completed assembly of the model without any fluid in the fluid domains.

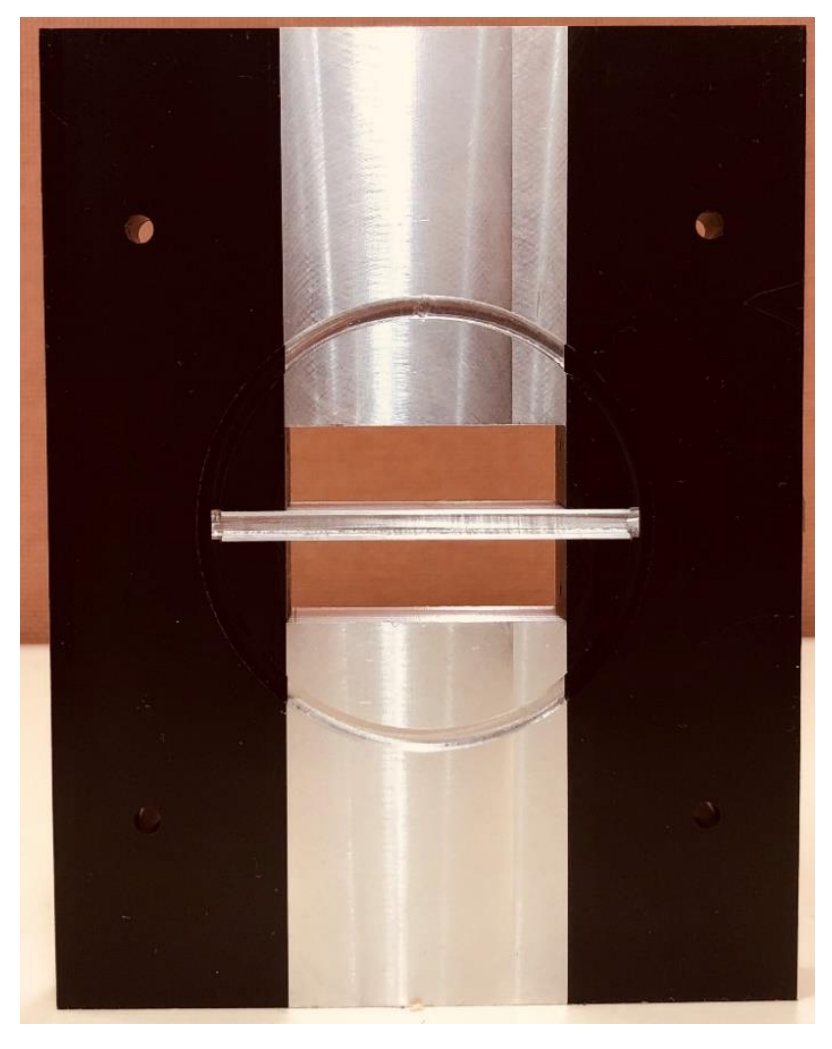

Figure 3.5: Half of the model assembled, without the O-rings in place. 


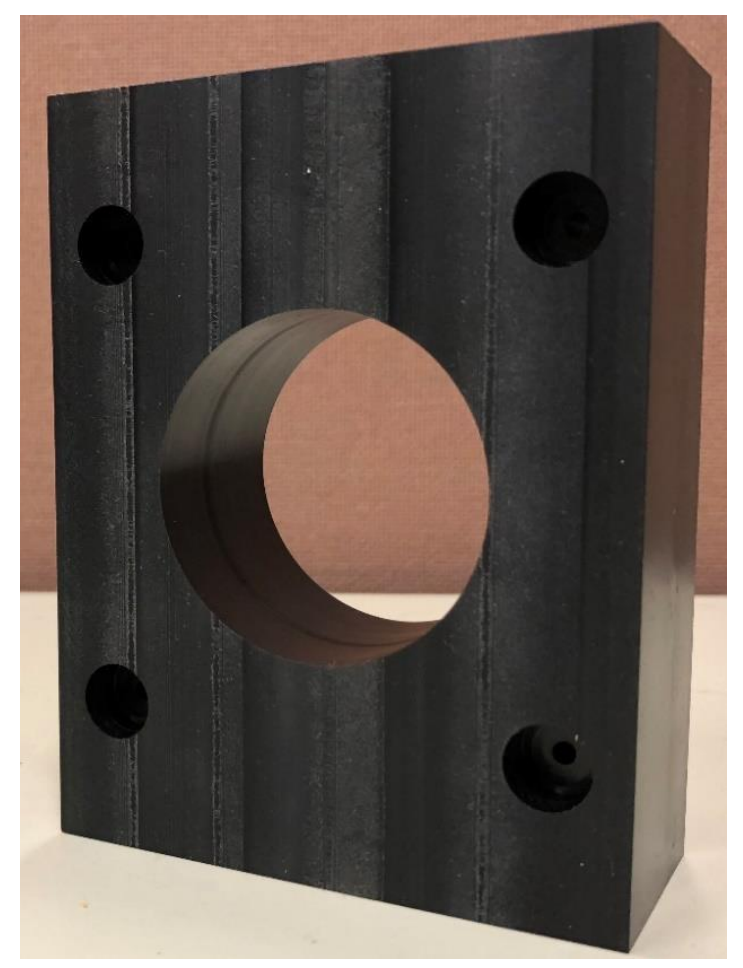

Figure 3.6: Isometric view of the optical frame.

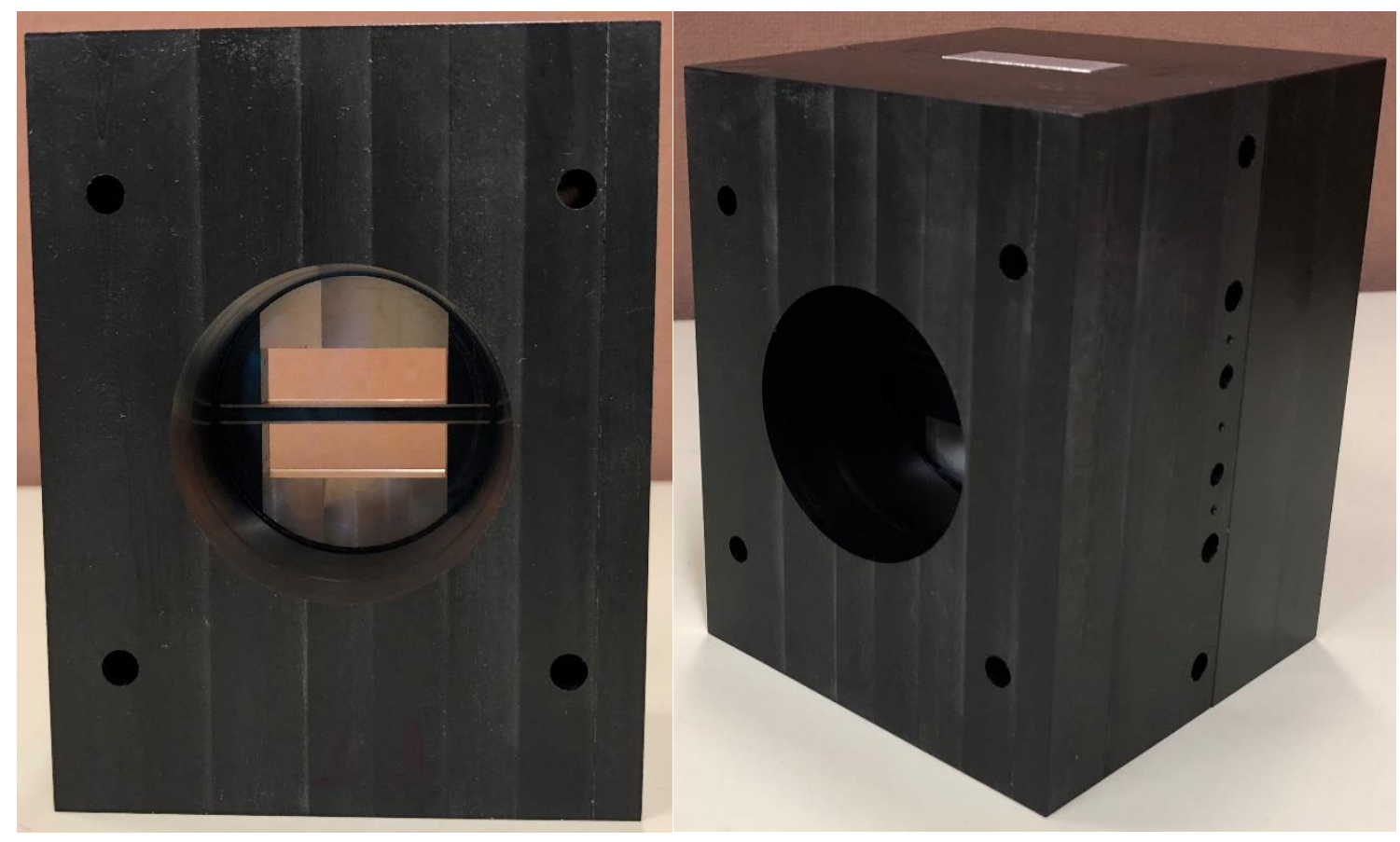

Figure 3.7: Assembled model (Front and Isometric view). 


\subsection{Experimental Procedure}

A well explained procedure of the experiment will be the purpose of the following section. The procedure will begin by setting up the interferometry, which was explained thoroughly by Roeleveld [2013], and will be referenced with a few minor adjustments. The process to assemble the model, inject the fluid, and the procedure to perform measurements that the desired temperature difference will be discussed further below.

\subsubsection{Sample preparation}

The procedure to suspend the nanoparticles within the nanofluid involves processing with an ultrasonic homogenizer. The homogenizer ensures a uniform distribution of nanoparticles within the base fluid (deionized water). As mentioned in Chapter 2, an industrially prepared nanofluid with a volume concentration of $20 \%$ was diluted with deionized water to prepare the lower volume concentration nanofluid needed for the experiment. The process of preparing the lower volume concentration of nanofluid was done using a calibrated digital scale, $50 \mathrm{ml}$ sample containers, deionized water and the industrial nanofluid. The procedure used to dilute the nanofluid to the desired volume concentration is given in Appendix E.3. Since the commercially prepared nanofluid was manufactured in terms of weight concentration, the conversion from weight concentration to volume concentration was done to dilute the sample solution for the experiment. Equation (3.1) was used to convert the weight concentration $(\omega)$ to volume concentration $(\phi)$ (refer to Appendix E.2 for a sample calculation of the conversion):

$$
\phi=\frac{\omega \rho_{w}}{\left(1-\frac{\omega}{100}\right) \rho_{n p}+\frac{\omega}{100} \rho_{w}}
$$

After the sample nanofluid of the desired concentration was prepared, the next stage was to sonify the locally prepared nanofluids. An ultrasonic processor UP400S (400 watts, $24 \mathrm{k} \mathrm{Hz}$ ) was used to suspend the nanoparticles. The sonification process is done by placing the container with the nanofluid within the ultrasonic processor's chamber with the probe submerged into the container. Approximately 45 minutes was spent sonifying the locally prepared nanofluid in the ultrasonic homogenizer, which showed physical signs of the nanofluids being evenly dispersed in the solution (no agglomerated particles on the bottom surface of the container). It was noted that the presence 
of an agglomeration of particles would occur about 24 hours after the solution was sonified. Thus, the procedure of sonifying the nanofluid was performed frequently.

\subsubsection{Model assembly}

Before the experiments could be started, the process of assembling the model was performed. There are six major parts that are required to be mated. This procedure required a lot of patience to ensure a tight fit was achieved for the fluid domains. Half of the model was already mated by the machinist, and the fasteners used in assembling half of the model should not be touched. The exposed surfaces of the fluid domain were cleaned with a microfiber tissue to remove any potential residue attached to the surface. The customized O-ring, with silicon lubricant applied to surface in contact with the optical window, was inserted into the designated groove. Once the O-ring was set in place, pressure was applied gently on the washer to remove excess lubricant. This was done to prevent the lubricant from smudging on the optical windows when the components were mated. The optical windows were gently placed into the optical frame slot and were aligned with the partially assembled model. With the components all aligned, the four fasteners were inserted and carefully tightened to assemble the model as one piece. It is important to note that tightening the nuts too far will cause the optical windows to bend. However, if the nuts are too loose, the fluid in the top domain will seep into the bottom fluid domain. At this point the model was fully assembled. It can be dissembled when working with different fluids for the experiment. The procedure should be done in reverse order when dissembling the model.

The next step with the model assembled was to inject the fluids into the fluid domain which was done in a unique procedure. Two pipettes were required to insert the liquid into the fluid domains of the model. One pipette was filled with deionized water. The second pipette was used to draw out the air from the model. The pipette holding the fluid was inserted into a hole on the right side of the model, and the second pipette was inserted on the opposite side. As the pipette with the fluid filled the fluid domain space, the pipette on the left side was released to allow the air within the fluid domain to be drawn out. This process prevented the interior of the fluid domain from increasing in pressure. This process was repeated for the bottom fluid domain. The goal of drawing out air was also to reduce the air bubbles trapped within the fluid domains. This was mainly the small air bubbles stuck to the surface of the barrier. 
The final step was to place the model within the supporting frame designed to secure the model in place and keep it leveled. The model should be placed at the center above plate 2, with the aluminum block (both hot and cold block) positioned at the center of the CPU cooling unit and the optical window facing in the direction of the flat mirror and beam splitter BS2 (Figure 2.5). With the model set in place, plate 1 was slid into place above the model and the nuts responsible for holding plate 1 were tightened. The digital level was placed on the surface of plate 2 and in front of the model. Based on the reading of the digital level, the three T-screwed were adjusted on the corners of plate 2 until the platform was leveled. Once plate 2 was leveled, the digital level was set on plate 1 , and the nuts were either be tightened or loosened to level plate 1 . At this point, the model was leveled. The thermocouples were inserted into the model with thermal paste applied to cover up the remaining hole.

A procedure that must be revisited while the model was leveled was the alignment of the second flat mirror and BS2 optical components. The process of the flat mirror and BS2 being adjusted to align the light to passing through the model in the test beam, must be redone after the model was

leveled. A reference that was used to align the flat mirror and BS2 was the light that reflected off the frame, plate 1 and 2 . The light reflection from the support frame was utilized to adjust the two optical components to obtain clear interference patterns.

\subsubsection{Experimental test run}

In this section, the process of gathering the data needed to measure the fringe gradient will be explained in a procedural manner. The process of taking the unheated and heated image will be explained and the process of calculating the relative thermal conductivity will be described.

The first image taken was the unheated case, where the temperature of both fluid domains and that barrier are at ambient conditions $\left(\sim 20^{\circ} \mathrm{C}\right)$. By doing so, the fringe pattern can be set and used to establish the temperature induced fringe shift when the model is heated to the desired temperature difference. Based on the thermocouple readings, if the temperature of the fluid and barrier were not at ambient conditions, the water baths were adjusted to reach the desired temperature. Once the temperature was set, the first data gathered was done using the finite fringe setting. The finite fringes were set for the unheated case such that there was a minimum of 8 fringes in both domains. 
These finite fringes were adjusted to be parallel to the barrier. The process of setting the finite fringes was done by adjusting beam splitter BS2. Once everything was set, the process of capturing the image using the digital camera was performed (refer to Appendix D).

The next step was to capture the fringe shift with the model at the desired temperature difference. The hot surface of the model was heated above room temperature, and the cold surface was cooled an equal amount below room temperature. This was done to minimize the heat transfer between the model and ambient air. The water baths were set to achieve the desired temperature difference, and to maintain a temperature of $20^{\circ} \mathrm{C}$ at the barrier. After 30 minutes had passed to allow the model to reach steady state, the thermocouples were checked to make sure the temperatures of the hot and cold plates are at the desired temperature difference. The barrier temperature was also checked. If they need to be changed, the water bath temperatures were adjusted until both desired temperature difference and desired barrier temperature was achieved. The number of fringes in both fluid domains was then checked. If the number of fringes present in both domains had roughly doubled, then the condition of the heated model was a success. Once again, an image of the model was captured on the camera, and the temperature difference, barrier temperature, and room temperature were recorded. This procedure was repeated with the unheated case in the infinite fringe setting and the model was reheated, and an image was captured.

\subsubsection{Processing data}

The process to extract the fringe gradient from the heated model was dependent on the image processing method performed on MATLAB Simulink. A detailed procedure to process the captured image is explained in Appendix D. The procedure to measure the numbers of pixels per fringe at specific location of each image is an iterative process, and the code written on MATLAB performs the iterative process. The image processing was performed for every captured image, as specific inputs are required to examine the area of interest. The algorithm was designed to measure

multiple locations along a concentrated area. The feature in this algorithm was beneficial to obtain a collection of data for each experiment performed in this project, because the average fringe gradient at the surface can be computed. 


\section{Chapter 4: Numerical Simulation of the Experimental Model}

\subsection{Introduction}

The focus of the chapter will be the information about computational fluid dynamics (CFD) and the numerical simulation of the experimental model. Numerical validation of an experimental method has become common practice in engineering research. The CFD analysis will provide an approximate solution to the proposed technique. The software that was used to produce a numerical solution of the problem was a combination of both COMSOL (two-dimensional analysis) and Flow Simulation module of SolidWorks (three-dimensional analysis). The purpose of the numerical study was to validate the technique and identify potential sources of error prior to the experimental study.

The CFD simulations will be performed with two different discretization methods, both capable of an approximate solution. The discretization method used by COMSOL is finite element method (FEM). SolidWorks uses the finite volume method (FVM). The difference in the techniques is how the domain is discretized when solving the governing equations. The FVM technique divides the computational domains into small control volumes and processes the conservations laws on the volumes. Whereas in the FEM, the computational domains are divided into many elements with the variables defined at points, referred to as nodes. In FEM, the weighted residuals of the governing equations are minimized over each element. However, both discretization methods will still yield similar results for the CFD problem.

The content of the chapter will begin by providing the details of the simplified model and the corresponding boundary conditions that were used in the CFD modelling. The governing equations for both two-dimensional and three-dimensional modeling will be explained. The primary interests in the analysis are the temperature distribution at the surface of the barrier that separates the nanofluid from the pure water, the variation of fluid temperature in the beam direction, and the continuity of the heat flux across the barrier. 


\subsection{Model geometry}

The model simulated in the numerical analysis is a simplified geometry which has excluded minor details such as extrusions and grooves. The study will demonstrate the results computed numerically using both steady-state 2D and 3D simulations. The two-dimensional analysis was computed with COMSOL, and the three-dimensional study was computed with SolidWorks. The simplified geometry essentially contains the frame of the experimental model with two fluid domains separated by a thin solid barrier. The nanofluids that will be used in the experiment are expected to have properties close to that of water. So, to simplify the modelling, both fluid domains were set to pure water.

Two-dimensional modelling was the primary procedure used to validate the technique, prior to the three-dimensional study. The two-dimensional study is capable of approximating the solution assuming heat losses in the third dimension would be small. Thus, the solution obtained in the twodimension study should yield roughly the same results as the three-dimensional study. The 2D study provides the temperature distribution throughout the entire model at steady state. The temperature distribution at the vertical center line of the model will be used to verify the proposed technique. This is where optical temperature measurements will be made in the experiment. Figure 4.1 shows the model simulated with COMSOL. In terms of dimensions, the thickness of the water $\left(\mathrm{th}_{\mathrm{w}}\right)$ layers in both upper and lower regions are $9 \mathrm{~mm}$, and the barrier thickness $\left(\mathrm{th}_{\mathrm{b}}\right)$ was $2 \mathrm{~mm}$. As for the datum for both $2 \mathrm{D}$ and $3 \mathrm{D}$ models, the origin of the coordinate system is located at the center of the barrier and the center of the overall width and depth of the geometry to simplify the results presented in the chapter. The geometry was used to verify the heat transfer in the fluid domains was conduction dominated. Table 4.1 lists the thermal conductivity of the materials used in the numerical simulations.

After performing the 2D study, a three-dimensional analysis was performed to ensure the experiment will perform as expected. In the 3D simulation, the temperature will change in all three coordinate directions ( $\mathrm{x}, \mathrm{y}$, and $\mathrm{z}$ ). The length of the fluid domain would have minimal heat loss in the z-direction, along with the width of the thin barrier. One of the concerns was to predict heat loss along both the $\mathrm{x}$ and $\mathrm{z}$ directions of the thin barrier. The depth ( $\mathrm{z}$ direction) of the fluid layers in the model will be maintained at $1 \mathrm{~cm}$, as mentioned in an earlier chapter. Figure 4.2 shows the 
geometry used for the three-dimensional analysis. Note that there were optical windows on each side of the model in the direction of the laser beam. Also, the model was insulated in all directions to prevent heat losses.

Table 4.1: Material properties.

\begin{tabular}{|c|c|c|}
\hline Component & Material & $\begin{array}{c}\text { Thermal Conductivity, } \\
\mathrm{W} /(\mathrm{m} \cdot \mathrm{K})\end{array}$ \\
\hline Housing & Acetal Homopolymer (Delrin) & 0.37 \\
\hline Barrier, Hot \& Cold Plate & Aluminum & 204 \\
\hline Optical Window & Glass & 0.5 \\
\hline Insulation & Foam & 0.020 \\
\hline
\end{tabular}

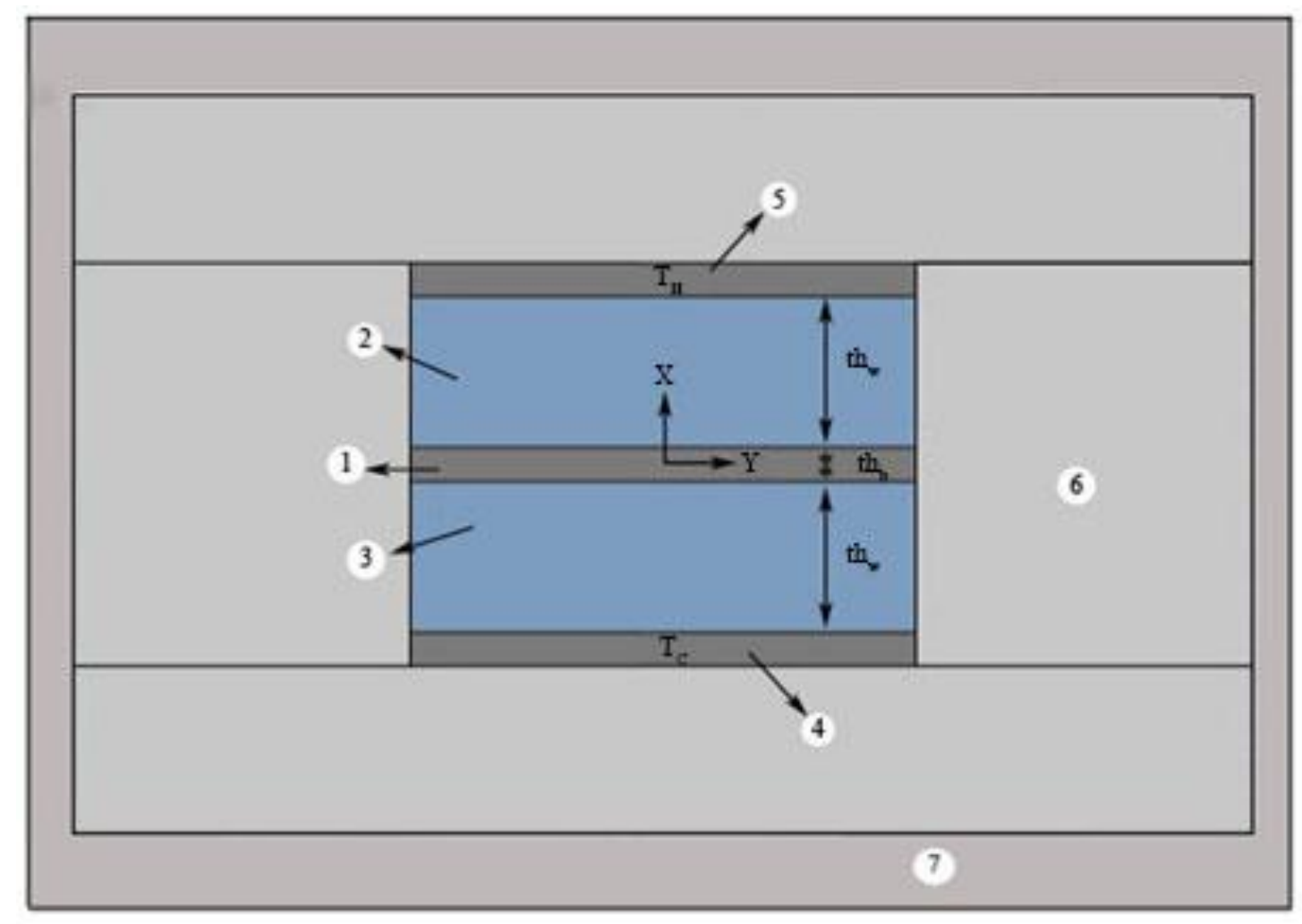

Figure 4.1: Sketch of the 2D model: (1) aluminum thin barrier, (2) \& (3) water fluid domains, (4) cold plate, (5) hot plate, and (6) housing., and (7) insulation. 


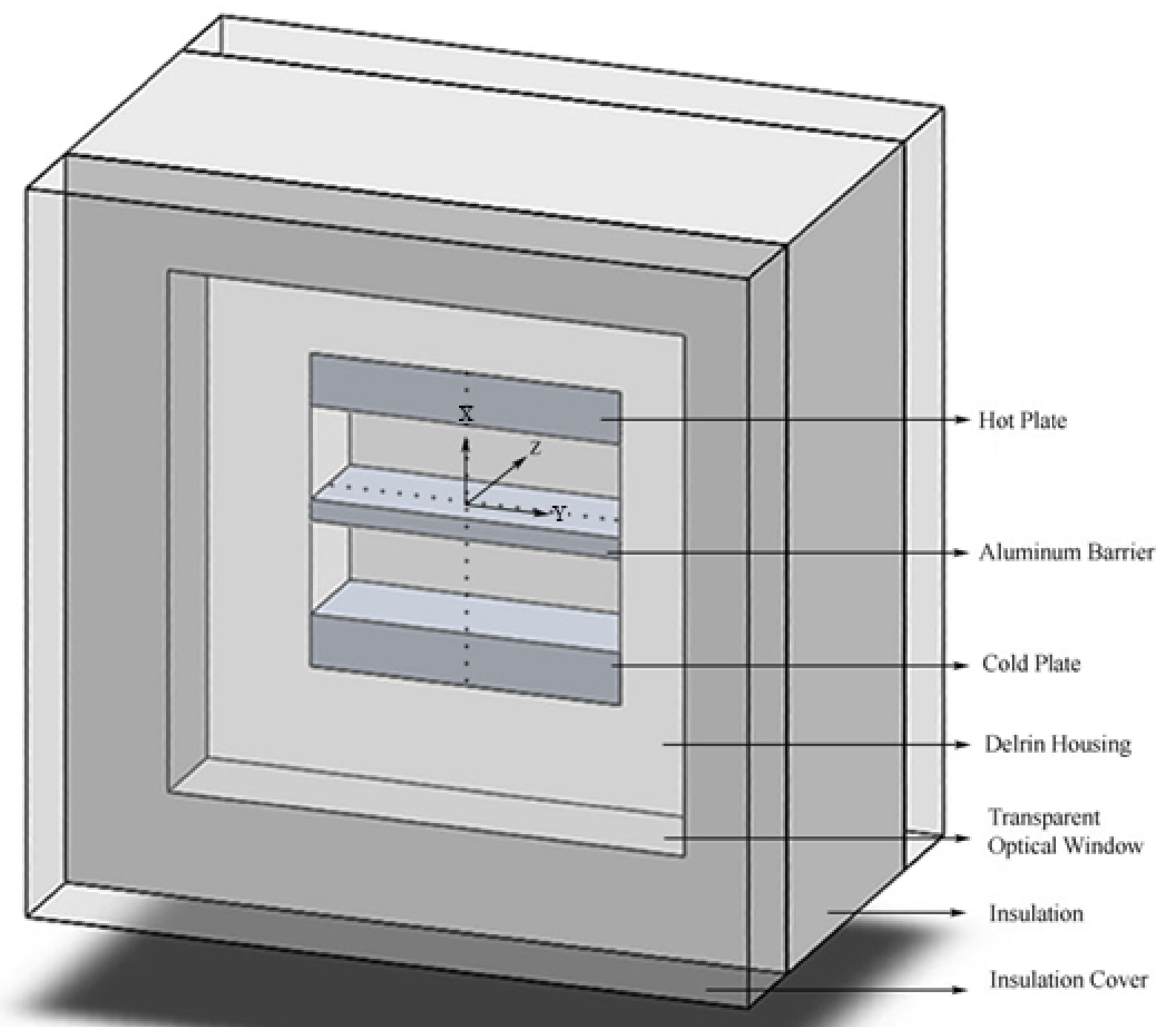

Figure 4.2: 3D model in SolidWorks.

There are a few applied boundary conditions that were required for the model in the numerical simulation. Table 4.2 are all of the assigned boundary conditions required for the numerical analysis. The value of the natural convection heat transfer coefficient used in the numerical study is based on the assumption that the experimented room temperature will be slightly below $20^{\circ} \mathrm{C}$, and the radiation from the surrounding was also considered. The heat transfer coefficient of the air is applied to the outer surfaces of the model. Aside from the properties of the materials of both solid and fluid domains, the main boundary condition that were required are the regulated temperatures (hot and cold plate) and the ambient temperature. 
Table 4.2: Boundary conditions applied.

\begin{tabular}{|c|c|}
\hline Boundary Condition & Value \\
\hline $\begin{array}{c}\text { Heat transfer coefficient for exterior convection } \\
\text { to the ambient }\end{array}$ & $10 \mathrm{~W} / \mathrm{m}^{2} \mathrm{~K}$ \\
\hline Hot plate surface temperature & $23^{\circ} \mathrm{C}$ \\
\hline Cold plate surface temperature & $17^{\circ} \mathrm{C}$ \\
\hline Ambient Temperature & $20^{\circ} \mathrm{C}$ \\
\hline
\end{tabular}

Due to the symmetry of the model, and the extensive computation time required to achieve a converged solution, the computational domain for the 3D model was set to be one quarter of actual model. By reducing the computational domain because of the symmetrical shape, the computation time is greatly reduced. Figure 4.3 provides an illustration of the reduced computational domain (grey region) used in the analysis. The gradient of pressure, temperature and the fluid velocities were set to zero on lines a symmetry. Also, the fluid velocity normal to a line a symmetry was set to zero.

The importance of the mesh size within the computational domain and the impact on the completion time to yield a converged solution will be explained. The mesh size used in both FEA and FVM solver have the same operation, where each individual nodes or volume would be used to solve each of the governing equations associated to the problem. An iterative process will occur solving the governing equations simultaneously, in which every individual mesh would yield a precise solution based on the establish boundary conditions to the problem. Improving the mesh size within the computation domain would improve the convergence and accuracy of the solution. The issue however, by increasing the mesh size will also increase the time required to approach a solution. For instance, one of the computations which consisted of 33,422 finite volumes within the domain required 1 hour to complete the computation. When the mesh was tripled, the time required to reach a convergent solution was in 2 hours. However, the difference in the heat transfer rate from the solution was by $6.5 \%$. Which implies a sufficient amount of mesh could be 
established to reduce the time to achieve a convergence solution. In the 3D simulation, the solution had converged with 93,180 finite volumes in shortest period of time. The heat transfer rate along the $\mathrm{x}$-direction was approximately $0.01 \mathrm{~W}$. Thus, the mesh sensitivity was an important procedure to achieve a precision solution and reduce the time required to achieve a converged solution.

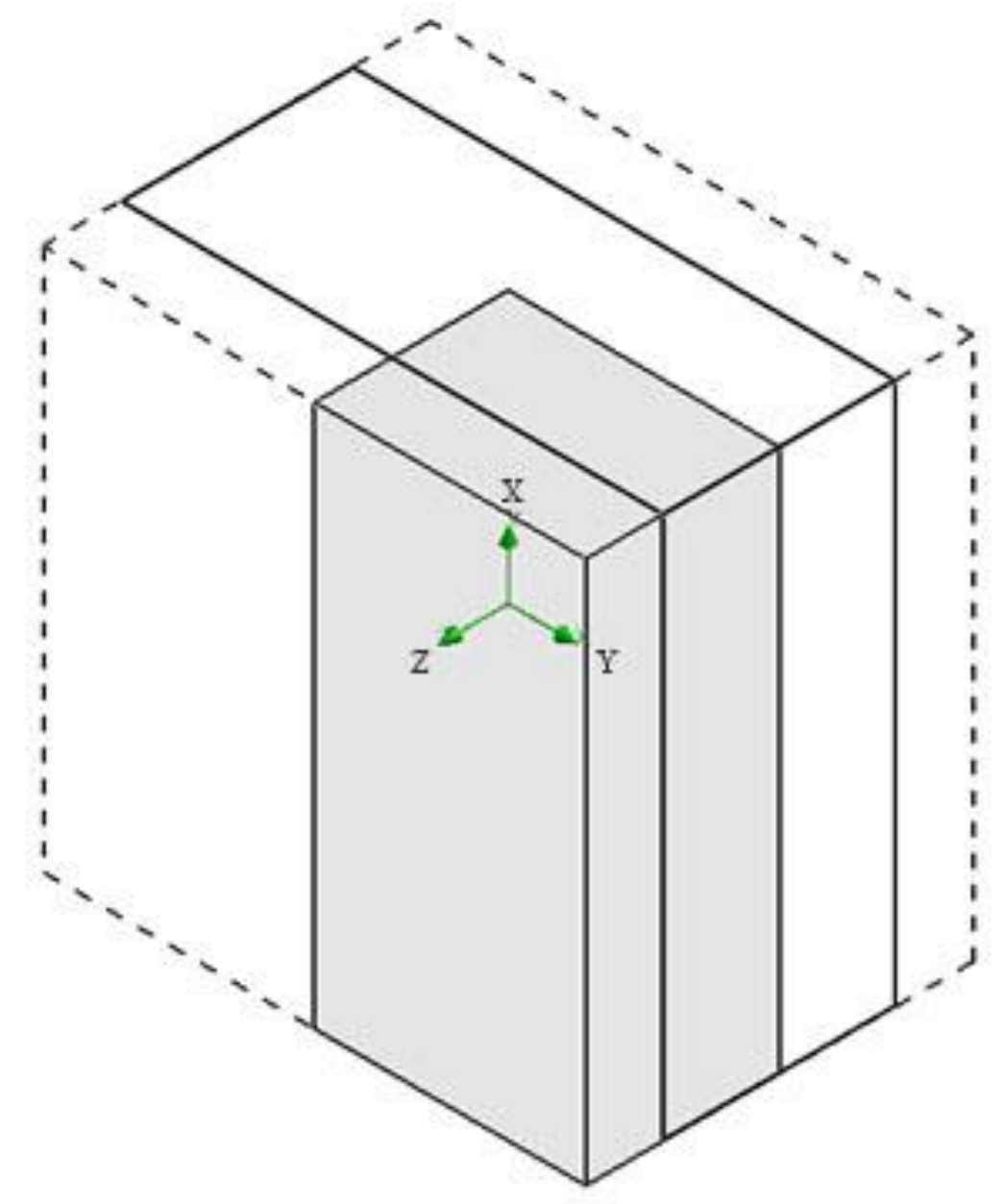

Figure 4.3 Reduced computational domain due to symmetric geometry and boundary conditions. The shaded section is the computational domain. 


\subsection{Governing equations and boundary conditions}

The results of the numerical solution were achieved through numerous iterations, all governed by the three fundamental physics equations. The governing equations that will be presented are specifically for fluid and heat transfer problems. The governing equations are the conservation of mass, energy and momentum. The Naiver Stokes equations are the momentum equations for CFD problems. A summary of the governing equations will be explained to understand the process within the CFD analysis and the results of each investigated element from the numerical solution will be discussed.

The boundary conditions assigned to the model are essential elements for the governing equations to yield a solution. There are three common boundary conditions in a CFD problem: Dirichlet, Neumann and Robin boundary conditions. The Dirichlet condition is defined as when a variable on a specific boundary is a known constant, which in the current simulation is the surface temperatures, $\mathrm{T}_{\mathrm{H}}$ and $\mathrm{T}_{\mathrm{C}}$. Location of the surface temperature are represented in equation (4.1) and 4.2:

$$
\begin{gathered}
T=T_{H} \text { at } y=\frac{t h_{b}}{2}+t h_{w} \\
T=T_{C} \text { at } y=-\frac{t h_{b}}{2}-t h_{w}
\end{gathered}
$$

The Neumann condition specifies the derivative of a variable along the boundary of the domain. In the current simulation, the velocity gradient and temperature gradient that are constant value (equal to zero) along lines of symmetry in the computational domain. Lastly, the Robin boundary condition is the combination of both Dirichlet and Neumann boundary condition. In the current simulation, the Robin condition is used to apply the convective boundary condition on the outside surfaces of the computational domain. This condition is defined by the heat transfer coefficient value $(\mathrm{h})$ and ambient temperature $\left(\mathrm{T}_{\infty}\right)$. This boundary condition is expressed by equation (4.3):

$$
-\left.k \frac{\partial T}{\partial n}\right|_{s}=h\left(T_{s}-T_{\infty}\right)
$$


where $\mathrm{n}$ is the unit normal vector to the boundary of the computational domain, and $\mathrm{T}_{\mathrm{s}}$ is the local surface temperature.

\subsubsection{Conservation of Mass}

The conservation of mass equation, otherwise known as the continuity equation, governs the mass flow over time in the problem. In the current problem, an incompressible fluid will be used, which means that density will remain constant in the continuity equation. The unsteady state incompressible continuity equation is:

$$
0=\nabla \cdot(\overrightarrow{\mathrm{V}})
$$

\subsubsection{Momentum equations}

The momentum equation is simply derived from the physical principle from the Newton's second law, and is shown in equation (4.5) (White, 1999). The equation is an expression that explains the moving fluid in the flow model, which considers both body forces and surface forces on a fluid element acting on all directions ( $\mathrm{x}, \mathrm{y}$, and $\mathrm{z}$ ). The flow is assumed to be laminar. The surface forces are the shear forces, normal stress forces and pressure distribution acting on the surface. Equation (4.6) - (4.8) represent the momentum equations in all three direction:

$$
\rho\left[\frac{\partial \vec{V}}{\partial t}+(\vec{V} \cdot \nabla) \vec{V}\right]=\rho g+\nabla \mathrm{p}+\mu \nabla^{2} \vec{V}
$$

X - Momentum:

$$
\rho\left(\frac{\partial u}{\partial t}+u \frac{\partial u}{\partial x}+v \frac{\partial u}{\partial y}+w \frac{\partial u}{\partial z}\right)=\rho g_{x}-\frac{\partial p}{\partial x}+\mu\left(\frac{\partial^{2} u}{\partial x^{2}}+\frac{\partial^{2} u}{\partial y^{2}}+\frac{\partial^{2} u}{\partial z^{2}}\right)
$$

Y - Momentum:

$$
\rho\left(\frac{\partial v}{\partial t}+u \frac{\partial v}{\partial x}+v \frac{\partial v}{\partial y}+w \frac{\partial v}{\partial z}\right)=\rho g_{y}-\frac{\partial p}{\partial y}+\mu\left(\frac{\partial^{2} v}{\partial x^{2}}+\frac{\partial^{2} v}{\partial y^{2}}+\frac{\partial^{2} v}{\partial z^{2}}\right)
$$

Z - Momentum: 


$$
\rho\left(\frac{\partial w}{\partial t}+u \frac{\partial w}{\partial x}+v \frac{\partial w}{\partial y}+w \frac{\partial w}{\partial z}\right)=\rho g_{z}-\frac{\partial p}{\partial z}+\mu\left(\frac{\partial^{2} w}{\partial x^{2}}+\frac{\partial^{2} w}{\partial y^{2}}+\frac{\partial^{2} w}{\partial z^{2}}\right)
$$

\subsubsection{Energy equation}

There are many forms of writing the energy equations. However, since the current problem is for an incompressible Newtonian fluid, the energy equation can be written as shown in equation 4.9, where $\boldsymbol{\varphi}$ is the dissipation function that accounts for the work done by the viscous forces. The variable $c_{p}$ is the specific heat at constant pressure. Note that for incompressible flows $c_{p}$ is almost equal to the specific heat at constant volume $\left(c_{v}\right)$.

$$
\rho c_{p}\left[\frac{\partial T}{\partial t}+(\vec{V} \cdot \nabla) T\right]=k \nabla^{2} T+\boldsymbol{\varphi}
$$

\subsection{Temperature profile}

The temperature distribution of the model is one of the main concerns of the validation analysis. The temperature distribution along the $\mathrm{x}$ direction has been calculated at steady state. There will be two temperature profiles examined in the procedure, one from both two and three dimensional study. The temperature profile will provide the temperature gradient in both the fluid and solid domains and can be used to validate the proposed technique. The temperature profiles developed from the CFD model will provide a clear understanding of the expected results from the experiment.

In this section, the results of the temperature profiles are examined and discussed. The CFD solution from the two-dimensional study has demonstrated the technique will work as expected. As shown in Figure 4.4, the upper and lower fluid domains have been predicted to have a linear vertical temperature distribution gradient. The reason the temperature gradient is linear throughout the entire region is because the buoyancy driven flow is very weak. The temperature is changing in both directions, however, the problem is close to a one dimensional, i.e., thermally stratified.

The linear temperature profile validates the technique will work as expected. The CFD model predicts that the heat transfer throughout the fluid layers will be conduction dominated. The next procedure will be to move to the three-dimensional study, which will incorporate the heat losses in the $\mathrm{z}$ direction. Figure 4.5 shows the temperature profile from the three-dimensional study. The 
important point from Figure 4.5, is to ensure a linear temperature profile is present along the surface of the thin barrier in the $\mathrm{x}$ direction. Figure 4.6 focuses on the temperature gradient near the surface and demonstrates a constant temperature gradient. The data from Figure 4.6 can be used to validate the performance of the technique by using equation (2.14). In the numerical solution, the water defined in the fluid domains were assigned the property of water. The thermal conductivity of the water at the top domain at the surface of the barrier was found to be equal to $0.594 \mathrm{Wm}^{-1} \mathrm{~K}^{-1}$. Using Figure 4.6, the temperature gradient was computed and substituted into equation (2.14) to determine the thermal conductivity of the water in bottom fluid domain. The computed thermal conductivity of the water in the bottom fluid domain was $0.574 \mathrm{Wm}^{-1} \mathrm{~K}^{-1}$. The percent difference between the thermal conductivity was computed to be equal to $3.50 \%$. Thus, this comparison demonstrates that this technique is capable of measuring the thermal conductivity of the nanofluids experimentally.

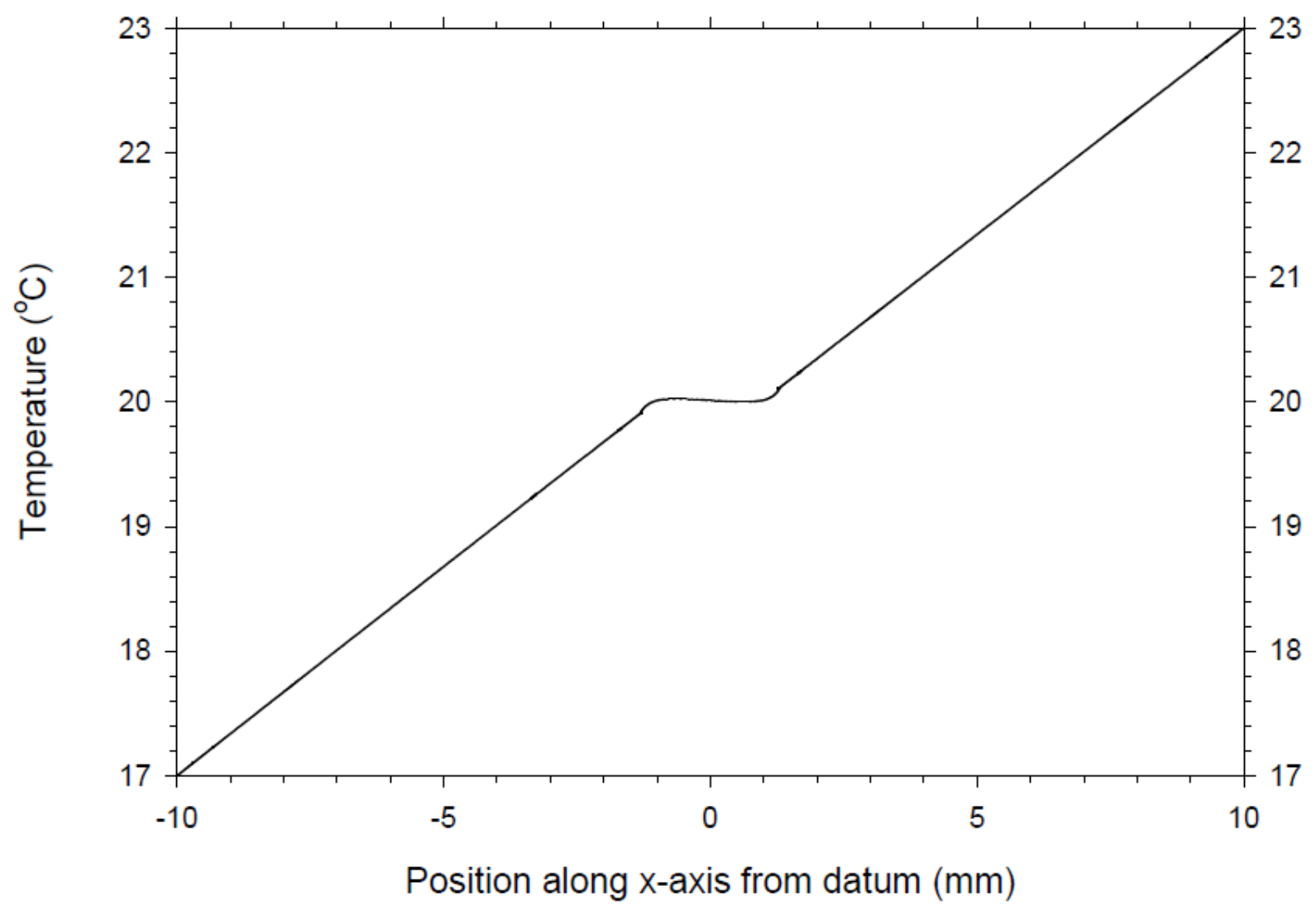

Figure 4.4: Two-dimensional temperature profile along the center line of the enclosure. 


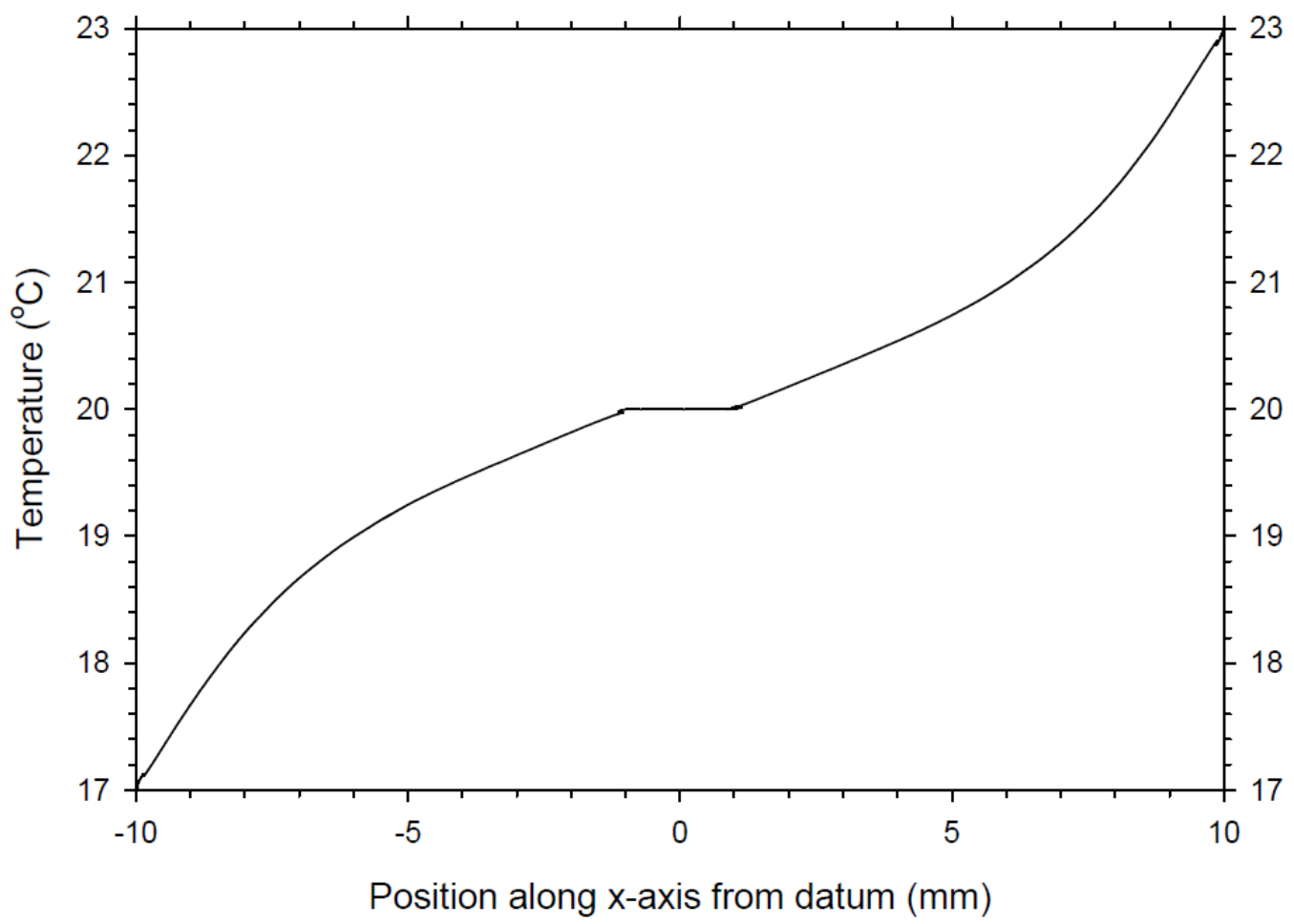

Figure 4.5: Vertical temperature profile on the center line of the model from 3-D study.

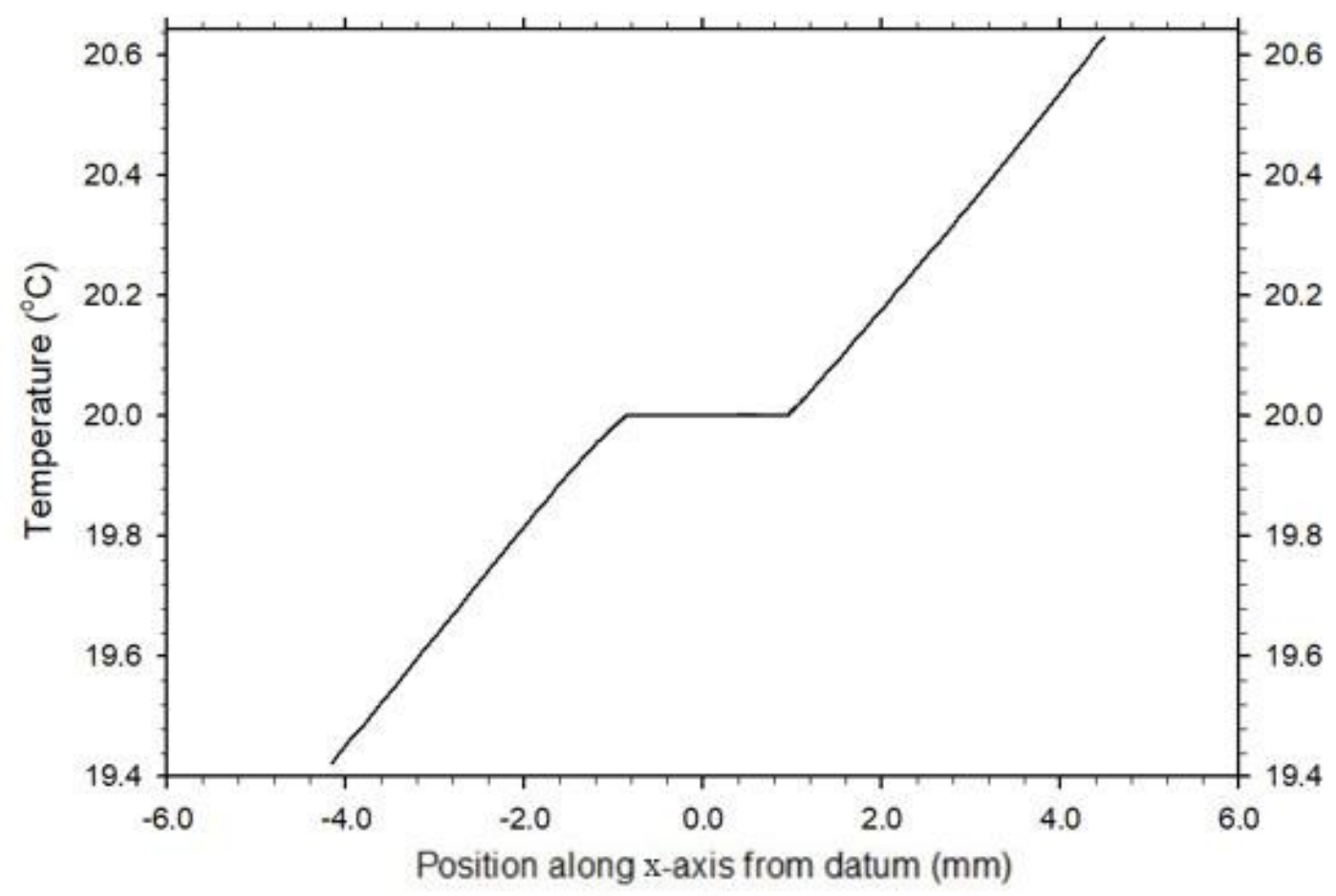

Figure 4.6: Vertical temperature profile near the barrier from 3-D study. 
Table 4.3: Percent difference in predicting the lower fluid domain's thermal conductivity at $20^{\circ} \mathrm{C}$.

\begin{tabular}{|c|c|c|}
\hline Predicted k value & $\begin{array}{c}\text { k value used in Numerical } \\
\text { Solution }\end{array}$ & Percent Error \\
\hline $0.574 \mathrm{Wm}^{-1} \mathrm{~K}^{-1}$ & $0.594 \mathrm{Wm}^{-1} \mathrm{~K}^{-1}$ & $3.50 \%$ \\
\hline
\end{tabular}

Figure 4.6 shows the temperature distribution close to the aluminum barrier. This graph shows the temperature profile in the region of the first two interference fringes, which will be used to calculate the temperature gradient. The graph demonstrates a nearly constant temperature gradient, which validates the temperature field that will be used near the barrier is dominated by conduction heat transfer.

In addition, the velocity gradients from the three-dimension study were investigated to understand the level of convection within the model. Figure 4.7 shows the velocity vectors and streamline within both fluid domains. The typical velocity was about $10^{-4} \mathrm{~m} / \mathrm{s}(\sim 0.1 \mathrm{~mm} / \mathrm{s})$. The figure shows some convection within the fluid domains, but the temperature field is strongly one-dimensional on the center line of the enclosure and conduction dominated. As well, the figure demonstrates the direction of the convection in both domains. 


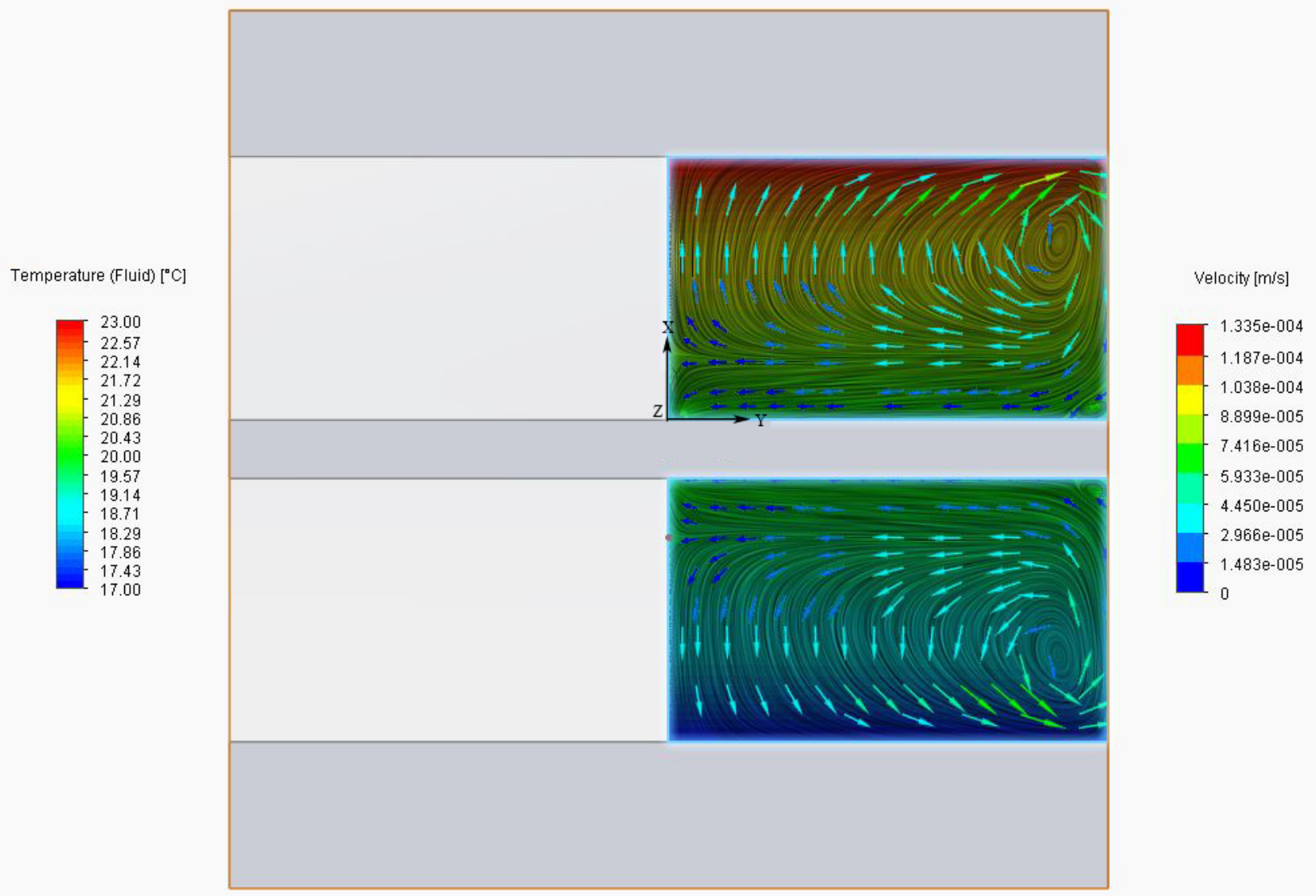

Figure 4.7: Velocity vectors and streamline superimposed over the temperature field from 3-D study.

\subsection{Temperature distribution along the beam direction}

The second important feature required to be investigated is the average temperature reading measured with the optical equipment. The fluid temperature measured by interferometry is an average temperature of the fluid along the path of the laser. The variation of the fluid temperature along the beam is an unknown factor and could potentially affect the accuracy of the measurements. For this reason, the temperature distribution along the beam direction was investigated with the numerical analysis. Since the optical window's diameter is $49.95 \mathrm{~mm}$, several locations within the path length along the center of the beam direction can be analyzed to understand the variation of the average temperature. Figure 4.8 shows the positions that will be analyzed to understand the variation of the temperature. The variation of the average temperature gradient can be determined by investigating the temperature distribution along the beam direction. 


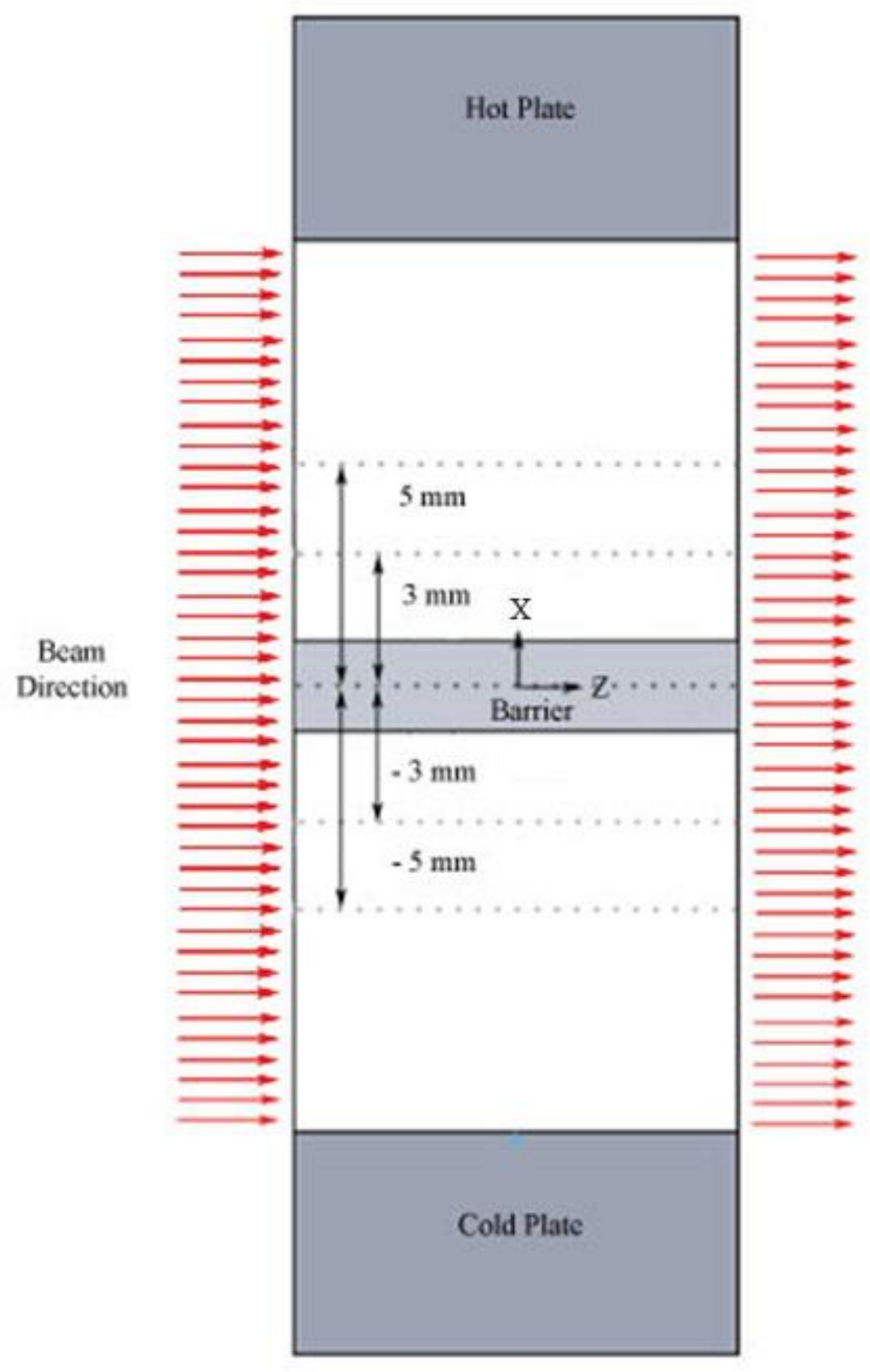

Figure 4.8: Cross section of the experimental model showing the line segments where the temperature variation in the beam direction has been plotted. 
Investigating the magnitude of the variation in the local temperature in the beam direction measured by the interferometry will be discussed. Based on Figure 4.8, Table 4.4 shows the fluctuation of the local temperature measured by the interferometry to be less than $0.1^{\circ} \mathrm{C}$. Figure 4.9 is the temperature distribution at $3 \mathrm{~mm}$ distance away from the datum along the $\mathrm{z}$-axis, and the fluctuation of the local temperature along the beam direction can be seen to be $0.04^{\circ} \mathrm{C}$. Table 4.4 demonstrates the fluctuation of the temperature distribution up to $\pm 4 \mathrm{~mm}$ from the barrier's surface. The maximum value was the highest fluid temperature that was located exactly at the center of the model, and the minimum value were located at the edges, adjacent to the optical window. Based on this data, the error in the interferometry measurements due to temperature variations in the beam direction should be very small.

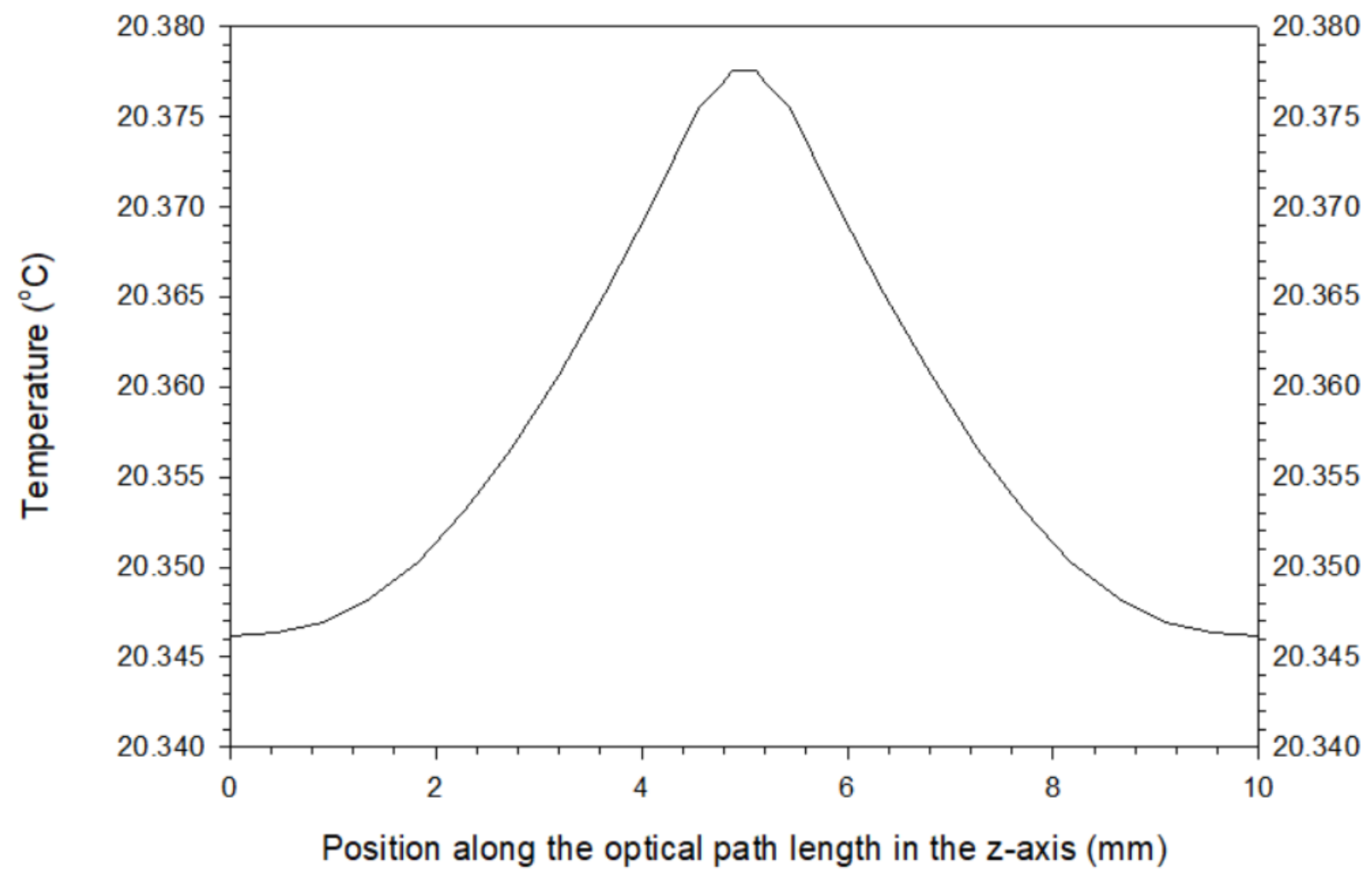

Figure 4.9: Temperature distribution in the beam direction at $3 \mathrm{~mm}$ away from the datum $\mathrm{x}$-axis 
Table 4.4: Fluid temperature distribution in the beam direction at varying $\mathrm{x}$-locations from the thin barrier.

\begin{tabular}{|c|c|c|c|}
\hline $\begin{array}{c}\text { Position } \\
\text { (x-axis) } \\
\text { (mm) }\end{array}$ & $\begin{array}{c}\text { Maximum } \\
\text { Temperature } \\
\left({ }^{\circ} \mathrm{C}\right)\end{array}$ & $\begin{array}{c}\text { Minimum } \\
\text { Temperature } \\
\left({ }^{\circ} \mathrm{C}\right)\end{array}$ & $\begin{array}{c}\text { Temperature } \\
\text { Variation } \\
\left({ }^{\circ} \mathrm{C}\right)\end{array}$ \\
\hline 5 & 20.74 & 20.68 & 0.06 \\
\hline 3 & 20.38 & 20.34 & 0.04 \\
\hline-3 & 19.64 & 19.62 & 0.02 \\
\hline-5 & 19.32 & 19.24 & 0.07 \\
\hline
\end{tabular}

\subsection{Heat transfer rate at the barrier}

The final important analysis is the heat transfer rate at the barrier. As discussed in Chapter 2, the theoretical concept of the technique is to use the Fourier's law at the surface of the barrier. The conduction heat flux in the $\mathrm{x}$-direction is assumed to be equal in the fluids on both sides of the barrier. Thus, it is important to check the heat transfer rate at the barrier with the numerical analysis.

The procedure to evaluate the heat transfer rate can be done by investigating the surface parameter of the thin barrier. Heat transfer rate on the top and bottom surface of the aluminum should be equal to validate equation (2.5). Table 4.5 shows the predicted heat transfer rate at the upper and lower surfaces of the barrier. The numerical solution has demonstrated that the heat transfer rate are equal, and that the proposed technique should give an accurate measurement of the thermal conductivity of the nanofluid. 
Table 4.5: Heat transfer rate at the barrier.

\begin{tabular}{|c|c|c|c|}
\hline $\begin{array}{c}\text { Upper Fluid Domain, } \mathbf{Q}_{\mathbf{x}} \\
(\mathbf{W})\end{array}$ & $\begin{array}{c}\text { Lower Fluid Domain, } \mathbf{Q}_{\mathbf{x}} \\
(\mathbf{W})\end{array}$ & $\begin{array}{c}\text { Barrier Side, } \mathbf{Q}_{\mathbf{y}} \\
(\mathbf{W})\end{array}$ & $\begin{array}{c}\text { Barrier Side, } \mathbf{Q} \mathbf{z} \\
(\mathbf{W})\end{array}$ \\
\hline $1.4 \times 10^{-3}$ & $1.4 \times 10^{-3}$ & $1.61 \times 10^{-5}$ & $4.86 \times 10^{-5}$ \\
\hline
\end{tabular}

The heat losses along the sides of the aluminum barrier were also investigated. Heat losses along the sides could potentially introduce minor errors into the measurements, since the method is based on one-dimensional heat transfer. The heat flux across the barrier in the $\mathrm{x}$ direction has been shown to be approximately constant, the heat losses in the $\mathrm{y}$ and $\mathrm{z}$ directions are shown to be extremely small. From the CFD analysis, the heat transfer rate along the y direction of the aluminum plate was calculated to be $1.61 \times 10^{-5} \mathrm{~W}$. This is much lower that the heat transfer rate in the $\mathrm{x}$-direction, indicating that side losses along the barrier are sufficiently small.

\subsection{Time dependent solution}

A 2D transient solution of the model was obtained, in order to have an estimate of the time required for the model to achieve steady state conditions. For the transient solution, the entire model was initially at room temperature $\left(20^{\circ} \mathrm{C}\right)$. At time zero, the desired temperature difference was applied to the heated and cooled surfaces of the model. The time step was 10 seconds. The solver was set to iterate until 20 minutes was achieved.

At the start of the experiment, after preparing the experimented nanofluids and water into the model. The process of extracting the measurement from the experiment are done only after steady state has been reached. However, the time required to reach steady state is an unknown factor, and can be determined by performing a time dependent analysis using the two dimensional study. Figure 4.10 demonstrates the time lapse of the experiment approaching steady state. The location of each analyzed position described in Figure 4.10 are relative to the datum along the x-axis. Based on the results, the time required to reach steady state will be about 10 minutes. 


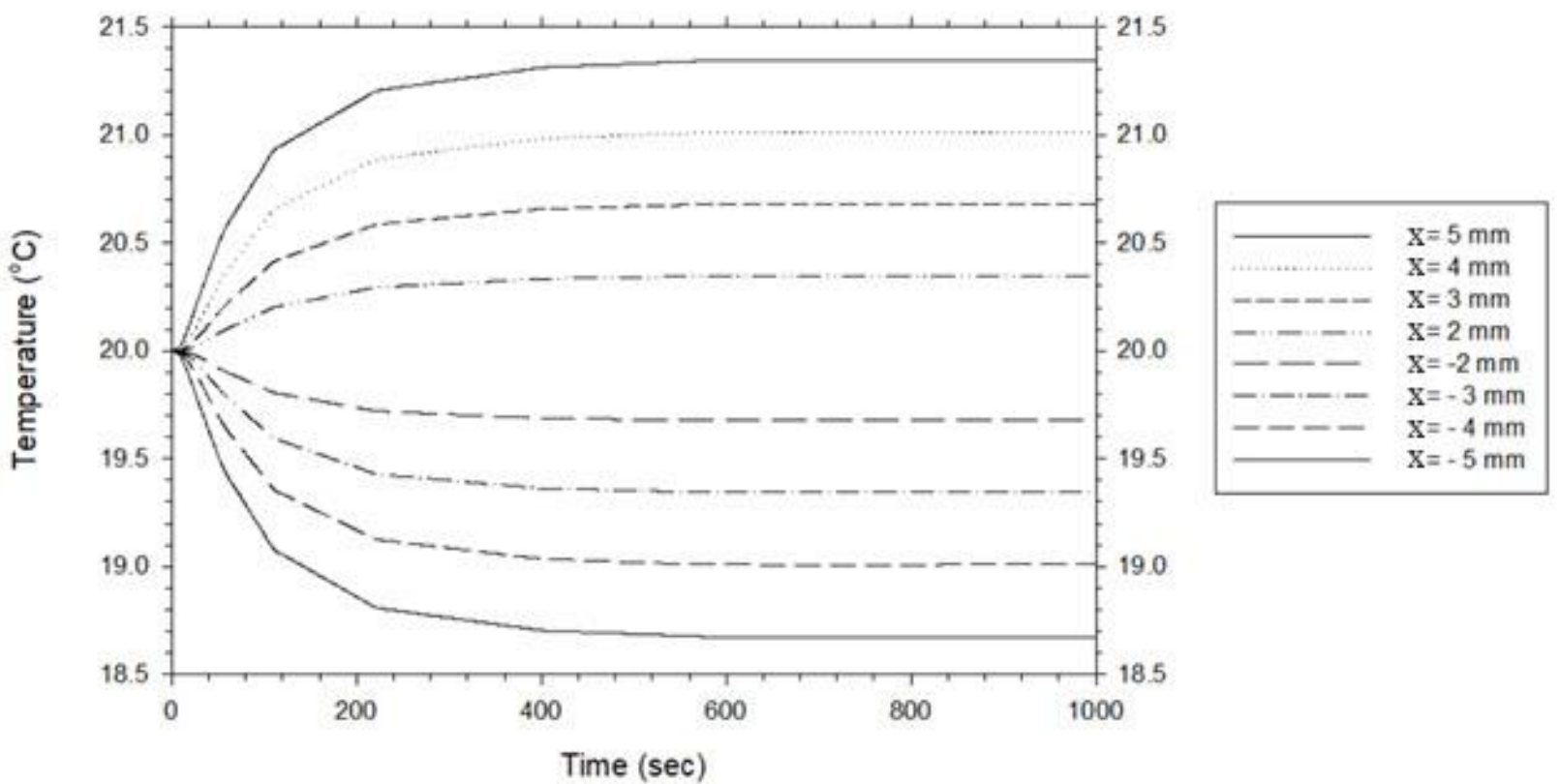

Figure 4.10: Time dependent solution of the temperature distribution in the experimental model from the datum. Temperatures are on the vertical center line of the model. 


\section{Chapter 5: Experimental Results and Analysis}

\subsection{Introduction}

This chapter reports laser-optical measurements of the thermal conductivity of a test fluid, made with the experimental model described in Chapter 3. In this model, the thermal conductivity of a test fluid is measured relative to a base fluid of known thermal conductivity. The results of a preliminary study are presented, which highlight some technical issues with the model. Results of experiments with water in both fluid domains are used to evaluate the method. Finally, the experimental method is applied to $\mathrm{SiO}_{2}$-water nanofluids.

\subsection{Preliminary experiment with nanofluid}

Some preliminary experiments were conducted to evaluate the performance of the model. These experiments revealed unexpected results. Figure 5.1 illustrates the main issues that were found. Figure 5.1 shows a finite fringe interferogram of the unheated model with $\mathrm{SiO}_{2}$-water nanofluid ( $10 \%$ by volume concentration) and water. With the interferometer in finite fringe mode, it was unexpected that the fringe gradient in the test fluid would be different from the base fluid (water) when no temperature difference was applied. As can be seen in Figure 5.1, the vertical fringe gradient in the nanofluid was approximately two times greater than in water. This unusual result required investigation to determine the possible reasons. The following two sections describe the analysis of this problem. 


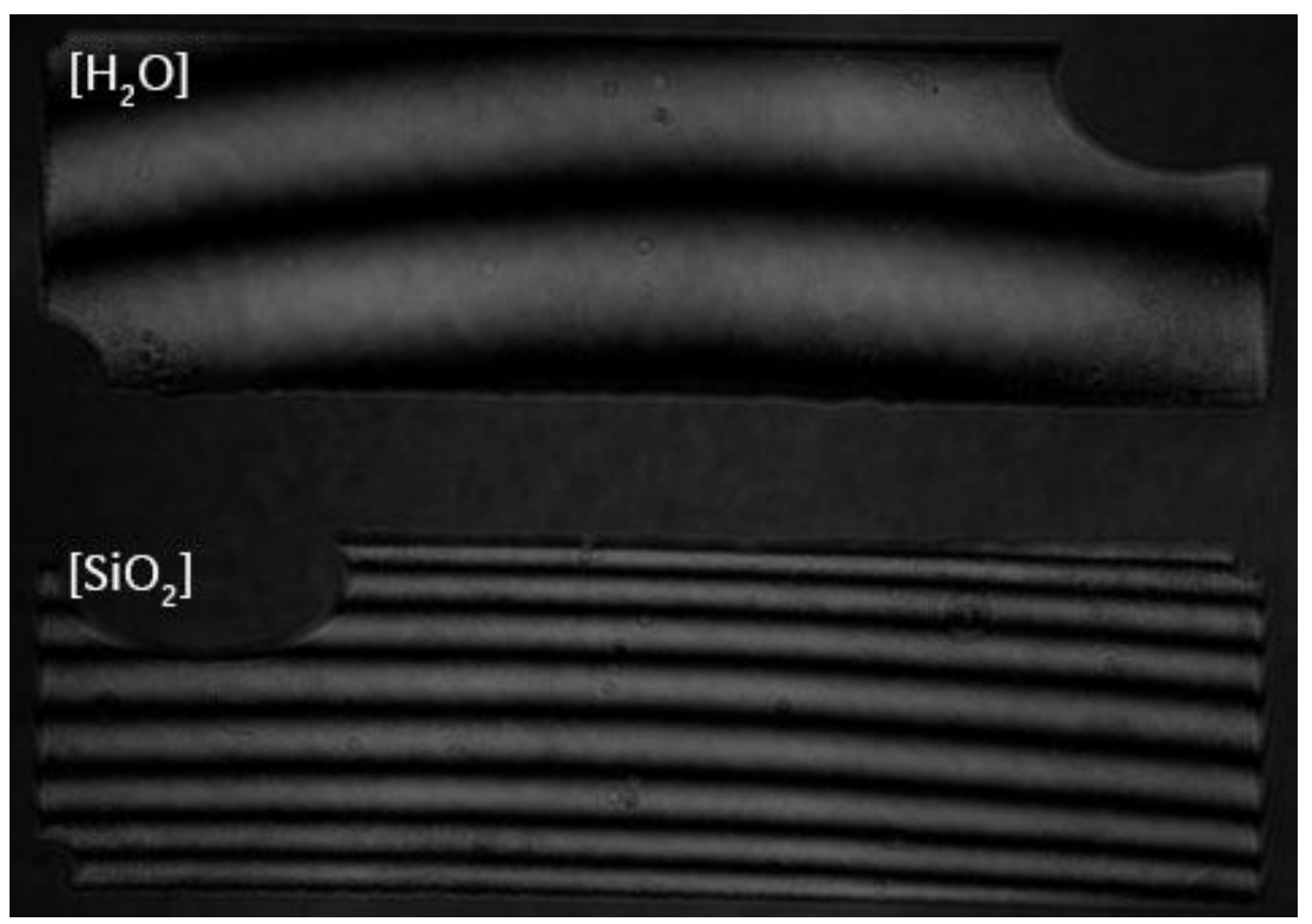

Figure 5.1: Finite fringe image of the unheated model with $\mathrm{SiO}_{2}$ - water nanofluid (10\% volume concentration) and water.

\subsection{Effect of non-parallel optical windows}

Laser interferometry measures very small changes in optical path of light. Due to the limitations of standard machining, as well as the way the windows are mounted in the model, the two optical windows will never be perfectly parallel. Non-parallel alignment in the optical windows will cause the path length to be greater in one fluid domain compared to the other. So, it is necessary to consider the effect of the non-parallel windows on the fringe gradient.

Figure 5.2 illustrates two non-parallel optical windows which are offset by a very small angle, $\theta$. As shown in this figure, the laser passes through a fluid with refractive index $n$. The angle between the vertical coordinate $(\mathrm{x})$ and each window is $\theta / 2$. Because the windows are not parallel, the laser passes through a greater thickness of fluid as $\mathrm{x}$ increases. 


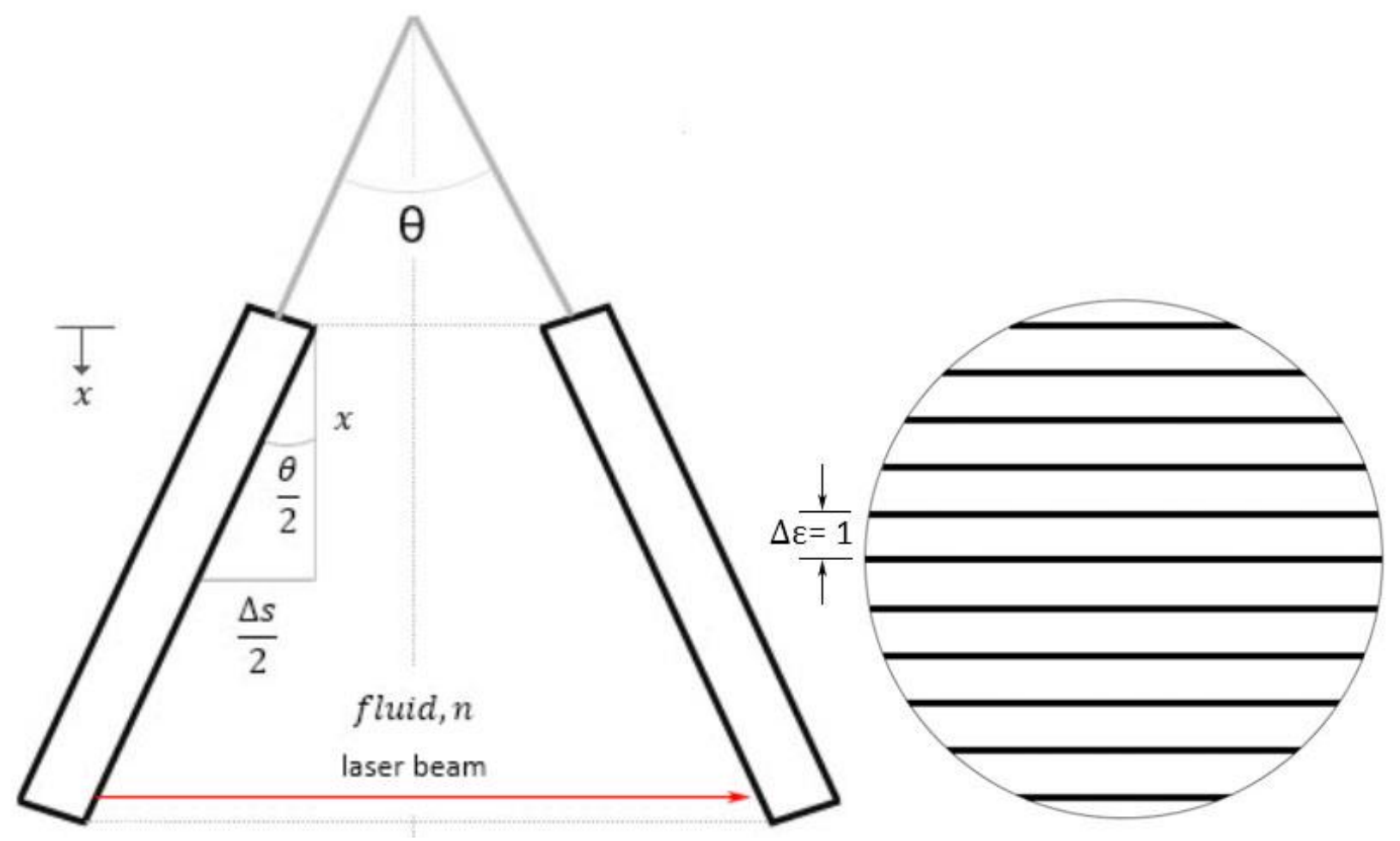

Figure 5.2: Misaligned optical windows.

From geometry, for small values of $\theta$ the small-angle approximation can be applied $(\tan \theta \approx \theta)$ and the additional path length $(\Delta s)$ through the fluid is given by:

$$
\Delta s=x \theta
$$

The additional path length produces a phase shift. The ratio of this phase shift to the wavelength of light in the fluid, gives an expression for the change in fringe order $(\Delta \varepsilon)$ in the $\mathrm{x}$-direction:

$$
\Delta \varepsilon=\frac{x \theta}{\lambda}
$$

Recalling that the wavelength of the laser light depends upon the refractive index of the fluid, as given in Equation (5.3):

$$
\lambda=\frac{\lambda_{o}}{n}
$$

where $\lambda_{o}$ is the vacuum wavelength of the laser. Substituting Equation (5.2) into Equation (5.3) gives an expression for the change in fringe order: 


$$
\Delta \varepsilon=\frac{x \theta}{\lambda_{o}} n
$$

Differentiating this equation with respect to $\mathrm{x}$, gives the following expression for the fringe gradient in the fluid, which is produced by the non-parallel optical windows:

$$
\frac{d \varepsilon}{d x}=\frac{\theta}{\lambda_{o}} n
$$

Equation (5.5) illustrates that the fringe gradient is dependent upon the refractive index of the fluid. Thus, when two different fluids are present between the same optical windows, some difference in the fringe gradients are expected.

Using Equation (5.6), in the current experiment the ratio of the fringe gradient in the nanofluid to the fringe gradient in the water will be given by:

$$
\frac{\left.\frac{d \varepsilon}{d x}\right|_{n f}}{\left.\frac{d \varepsilon}{d x}\right|_{w}}=\frac{n_{n f}}{n_{w}}
$$

In interferogram shown in Figure 5.1, the nanofluid with a volume concentration of $10 \%$ by volume has a refractive index of $\mathrm{n}_{\mathrm{nf}}=1.353$ [Huang et al., 2014] and the water has a refractive index of $n_{w}=1.33$. Equation (5.7) gives a fringe gradient ratio of:

$$
\frac{\left.\frac{d \varepsilon}{d x}\right|_{n f}}{\left.\frac{d \varepsilon}{d x}\right|_{w}}=\frac{n_{n f}}{n_{w}}=\frac{1.353}{1.333}=1.015
$$

Therefore, a $1.5 \%$ difference in the fringe gradient in the nanofluid and water is expected due to the effects of the non-parallel optical windows. Referring to the preliminary experiment shown in Figure 5.1, recall that the ratio of the fringe gradient in the nanofluid to the fringe gradient in the water is approximately 2.0. So, only a small portion of the addition fringes are caused by the nonparallelism of the window. Window non-parallelism is significant, but not the dominate effect. The next section examines another, more significant, effect.

As an aside, the results of this section show that, when working with two different fluids in the current model, there will always be a difference in the fringe gradients. As a result, it will not be possible to achieve the infinite fringe setting in both the base fluid and test fluid simultaneously. 
For this reason, with the current method, it will be necessary to use the finite fringe setting for experimental measurements involving two different fluids.

\subsection{Deflection in the optical window}

Another possible source of fringe gradient in an unheated model is the deflection of the optical windows in their mounts. The uneven tightness in the four fasteners used to mate the model, and the gaskets located between the aluminum barrier and windows have the potential to bend the optical windows. Even a small amount of bending will change the optical path $(\Delta s)$ and produce fringes. In this section, an estimate of the deflection due to the loading of the optical windows, and its impact on the measurements is evaluated. The problem is first analyzed as a simply supported beam, to determine the order of magnitude of the deflection caused by the gaskets. This highly simplified model is verified by comparing the results to three-dimensional finite element simulation.

\subsubsection{Approximation of the force}

To prevent leakage between the two fluid cavities, a customized O-ring gasket was located in a groove on both sides of the aluminum barrier. The O-ring was compressed against the face of the optical window along the window's center line. There was also an O-ring around the perimeter of each optical window. These compressed gaskets generate stress in the optical windows. So, the first step to determine the order of magnitude of the deflection in the optical window was to evaluate the magnitude of the force generated by the O-ring.

The compressive force between the O-ring and optical window was estimated by analyzing a reference image from an experiment performed in infinite fringe mode, shown in Figure 5.3. In acquiring this image, great care was taken to minimize the stress on the optical windows. The fasteners were tightened sufficiently to seal the enclosure and prevent leaks, and not torqued beyond this level.

In infinite fringe mode, with the same fluid on both sides of the barrier, the ideal image should not have visible fringes. The entire fluid field should have a single pixel intensity. However, this is not 
the case in the results from the experiment shown in Figure 5.3. In the vertical direction there is approximately one half a fringe shift in each fluid domain. This fringe shift is caused by the deflection of the optical windows and can be used to estimate the bending force. Figure 5.4 shows a vertical scan of the pixel intensity along the center line of the upper fluid domain. This graph confirms that the fringe shift from the edge of the upper fluid domain to the center of the barrier is approximately $\Delta \varepsilon=0.5$. This fringe shift can be used to estimate the maximum deflection present in the model, and hence the approximate bending force.

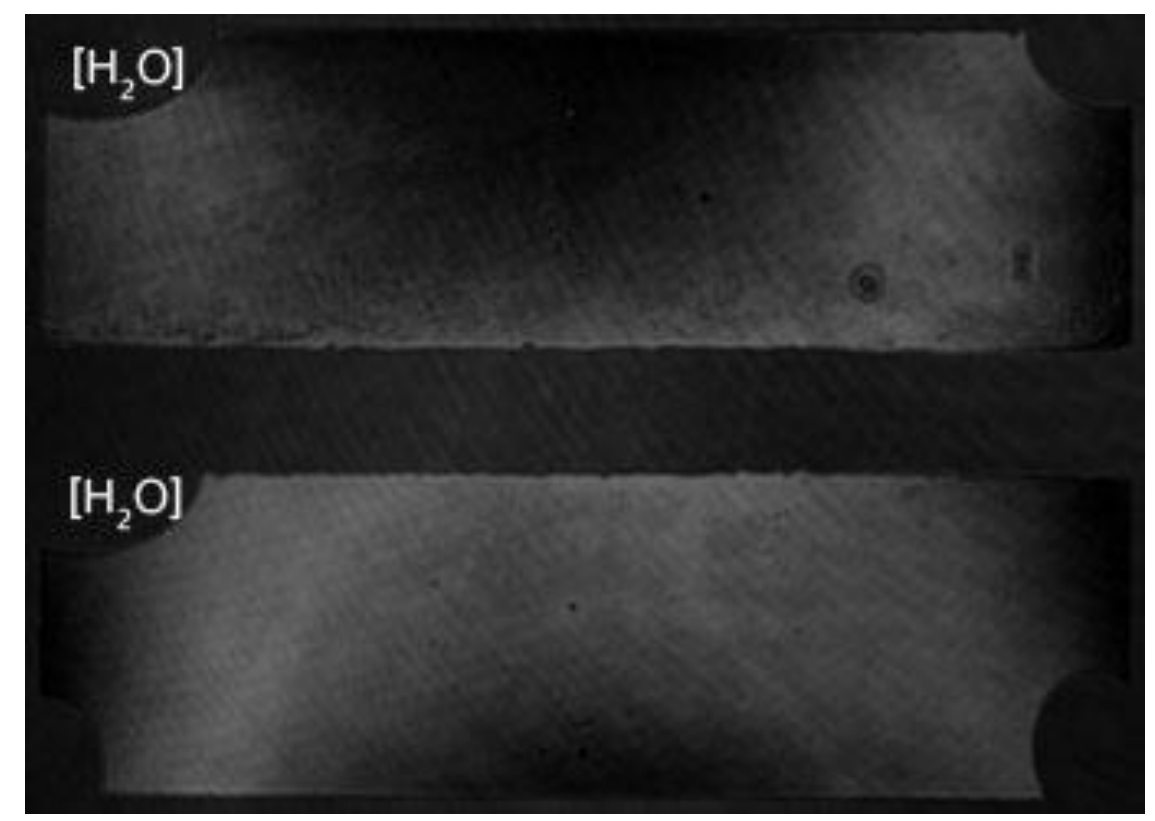

Figure 5.3: Unheated reference image in infinite fringe mode with water in both fluid domains.

As light passes through the water medium, the speed of light will change correspondingly. Equation (2.7) demonstrates the wavelength is inversely proportional to the refractive index of a medium. Using equation (2.7), the wavelength of the laser light will change to $474.7 \mathrm{~nm}$ as the light passes through the water in the fluid domain:

$$
\lambda=\frac{\lambda_{0}}{n}=\frac{6.328 \times 10^{-7} \mathrm{~m}}{1.333}=474.7 \mathrm{~nm}
$$




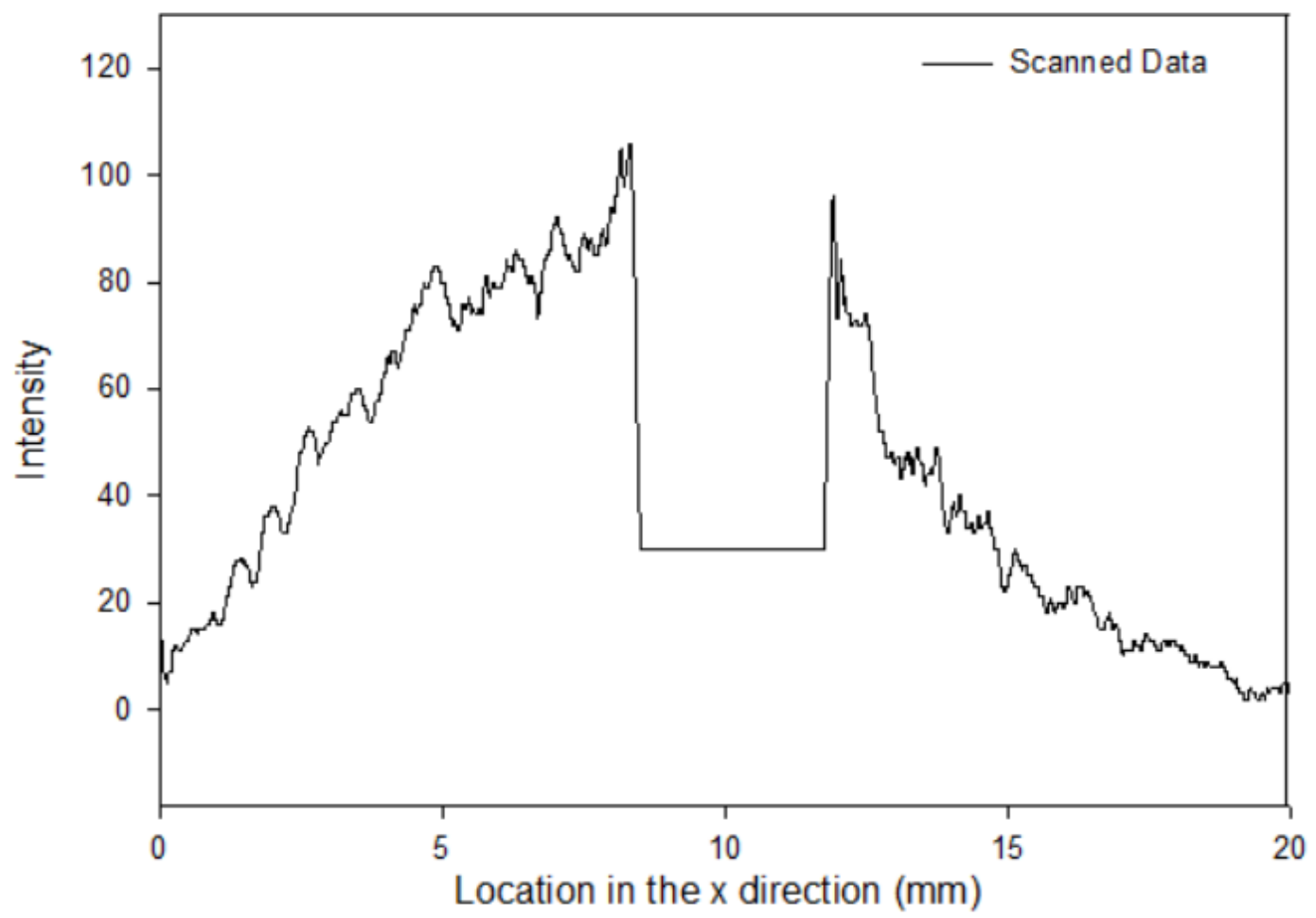

Figure 5.4: Plot of the pixel intensity with distance from the top of the upper fluid domain (for image shown in Fig. 5.3).

Based on the additional fringes ( $\Delta \varepsilon \sim 0.5$ ) seen in the reference image and the wavelength of the light passing through the water, the maximum deflection can be calculated as:

$$
\Delta \varepsilon=\frac{2 z_{d}}{\lambda_{w}}
$$

where $\mathrm{z}_{\mathrm{d}}$ is the maximum deflection in the center of one optical window. The factor of two in the numerator of Equation 5.9 is due to the fact that there is a deflection at both optical windows. The maximum deflection is calculated to be the following:

$$
z_{d}=\frac{\Delta \varepsilon \lambda}{2}=\frac{(0.5) \times(474.7 \mathrm{~nm})}{2}=1.189 \times 10^{-7} \mathrm{~m}
$$


Approximating the optical window as a simply supported beam is a first method used to establish an order of magnitude of the force causing the optical window to bend. Figure 5.5 illustrates the classical simply support beam problem. In this model, the compressive force $\left(\mathrm{F}_{\mathrm{ga}}\right)$ on the optical window from the gasket, is assumed to be a concentrated load along the center of the optical window. The Delrin frame holding the optical window in place was interpreted as simply supported boundary conditions. Although a crude model of the actual state of stress, this approach will give an estimate of the order of magnitude of the force between the window and gasket.
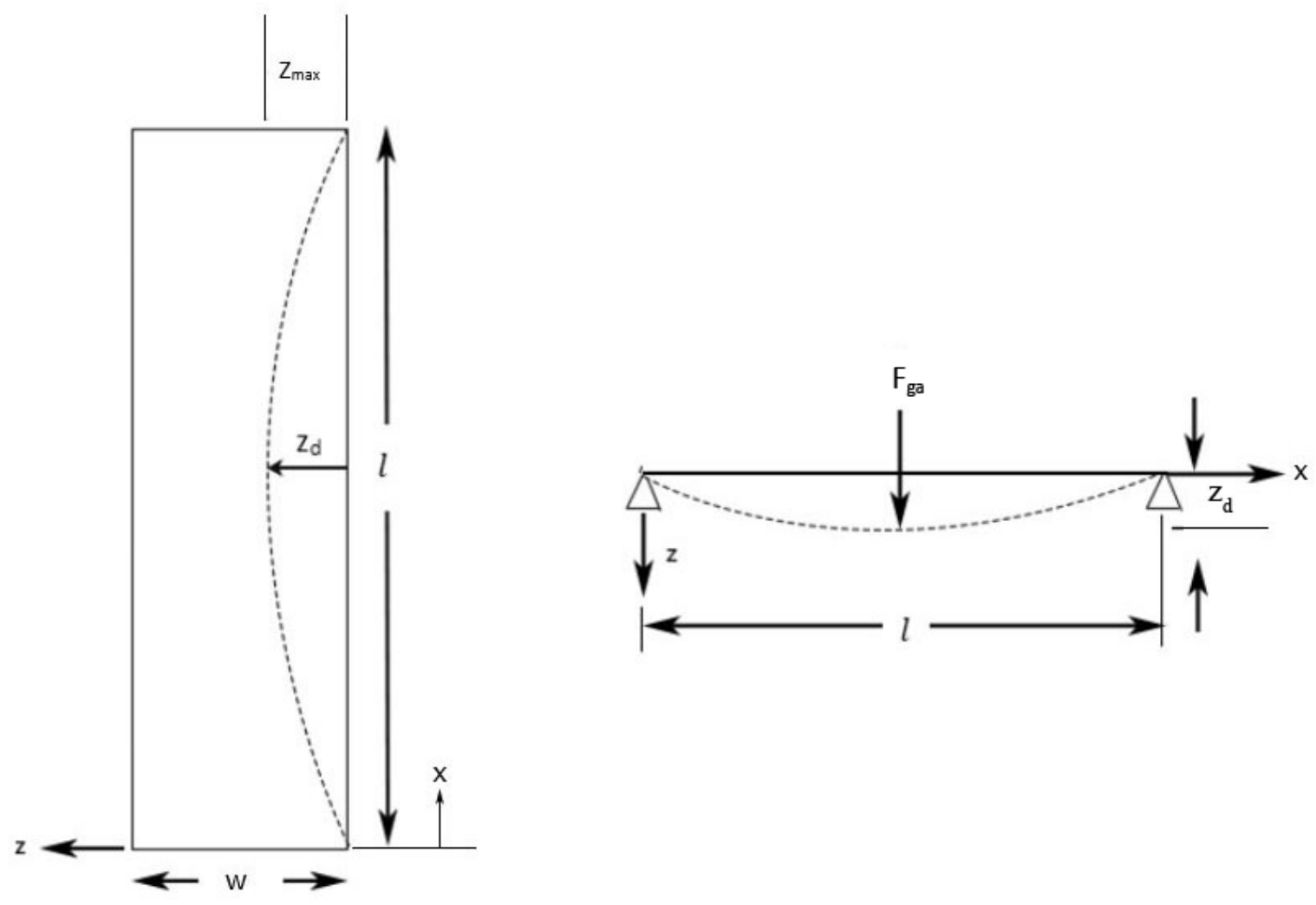

Figure 5.5: Optical window bending due to deflection (left) \& simply supported beam with concentrated load at the center (right).

To calculate the second moment of area, the optical window was approximated a rectangular geometry. The second moment of area of a rectangle was calculated using equation (5.10) and correspond to the variables shown in Figure 5.6. 


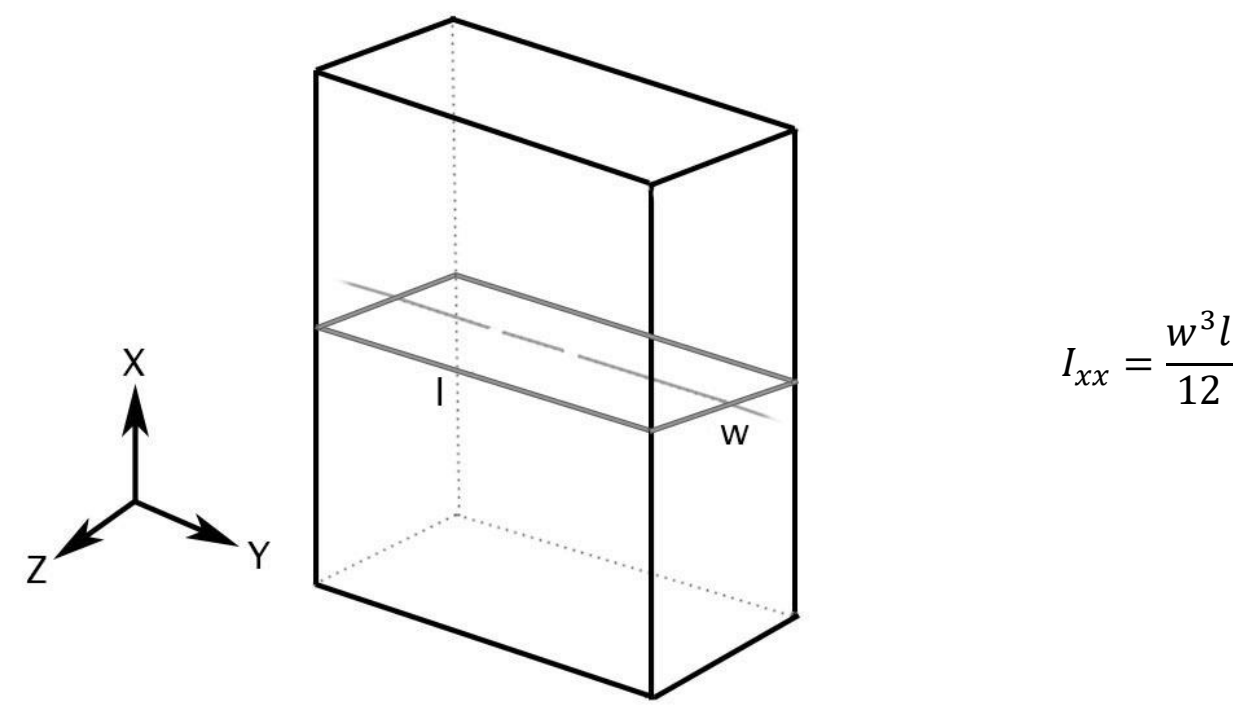

Figure 5.6: Rectangular geometry corresponding to the moment of inertia equation.

where $\mathrm{w}$ is the thickness of the optical window and 1 was taken as the diameter of the optical window.

Using the dimension of the optical windows ( $\mathrm{w}=4.25 \mathrm{~mm}, \mathrm{l}=\mathrm{D}=50 \mathrm{~mm}$ ), the second moment of area can be calculated as:

$$
I_{x x}=\frac{w^{3} l}{12}=\frac{(0.00425 \mathrm{~m})^{3} \times(0.05 \mathrm{~m})}{12}=3.20 \times 10^{-10} \mathrm{~m}^{4}
$$

The maximum deflection $\left(\mathrm{z}_{\mathrm{d}}\right)$ of a simply supported beam with a concentrated load at the center is expressed using Equation 5.11 [Beer et al., 2009]:

$$
z_{d}=\frac{F_{g a} l^{3}}{48 E I}
$$

The deflection calculated from the maximum number of fringes in the unheated image is the maximum deflection. Thus, the force caused by the gasket $\left(\mathrm{F}_{\mathrm{ga}}\right)$ can be solved by rearranging equation (5.11). The modulus of elasticity for fused silicon optical window is $73.1 \mathrm{GPa}$ [Crystran, 2012]. Rearranging equation (5.11) and solving for the force gives:

$$
F_{g a}=\frac{48 E I z_{d}}{l^{3}}=\frac{48\left(73.1 \times 10^{9} \frac{\mathrm{N}}{\mathrm{m}^{2}}\right)\left(3.20 \times 10^{-10} \mathrm{~m}^{4}\right)\left(1.189 \times 10^{-7} \mathrm{~m}\right)}{(0.05 \mathrm{~m})^{3}}=1.07 \mathrm{~N}
$$


This estimated compression force has been compared to the O-ring manufacturer's data, shown in Figure 5.7. Figure 5.7 gives the compression load on the silicon O-ring as a function of the "\% squeeze". The "\% squeeze" is interpreted to be the compressive strain, based on the O-ring diameter. The experimental model was designed to compress the O-ring to fill a square cavity by approximately $2 \mathrm{~mm}$. For a $1.6 \mathrm{~mm}$ diameter O-ring, this corresponds to $10 \%$ compression, if the O-ring was fully compressed. The length of the O-ring at the barrier is $3.0 \mathrm{~cm}$ (1.18 inches). Accounting for this length, and converting the force to metric units, the manufacturer's graph indicates that a force of approximately $1-5 \mathrm{~N}$ (equal to $1.14-5.65 \mathrm{lbs} / \mathrm{in}$ of O-ring circumference) is needed to compress the O-ring by $10 \%$. As previously discussed, fasteners were not fully tightened in this experiment (Fig. 5.3), and the O-ring was not likely fully compressed. Given this fact, and the approximate nature of the mathematical model, it is reasonable to conclude that the estimated force is consistent with the manufacturer's data. This analysis demonstrates that the compressive force of the O-ring on the optical window is sufficient to induce bending that is consistent with the measured fringe distortion.

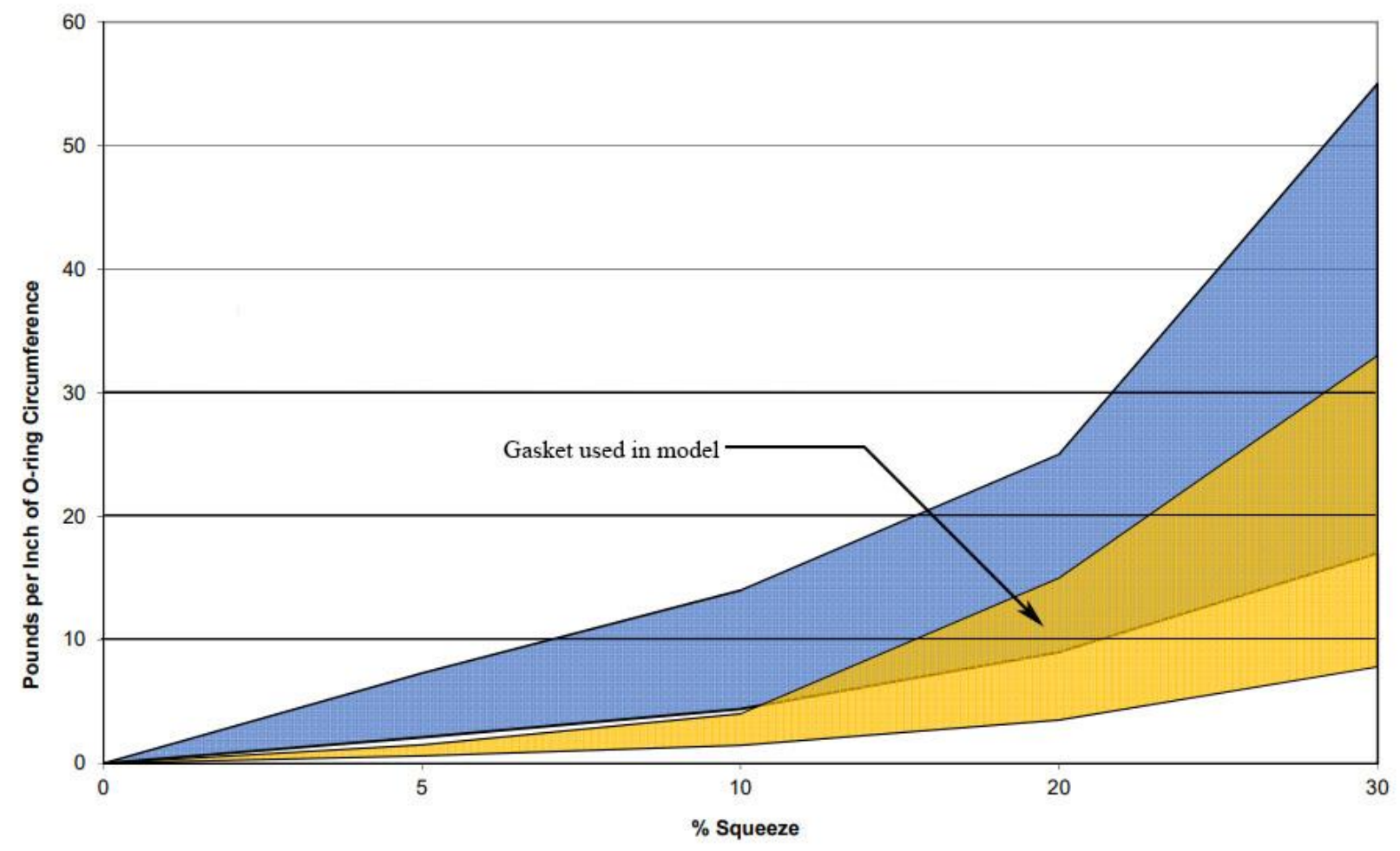

Figure 5.7: Compressive force (per unit circumference) versus compressive strain the silicon Oring (1.6 mm dimeter O-ring) (Parker, 2019). 


\subsubsection{Deflection approximation}

A correction to the measured fringe gradient can be approximated based on approximated force in the previous section's analysis. Based on Figure 5.1, the additional fringes that cannot be controlled due to the deflection in the optical window, can be corrected in the technique by computing the measured fringe gradient. By doing so, the measurement uncertainty of the experiment performed in infinite fringe mode would improve. The process to approximate the additional fringe gradient at the location of the measured data will be done by using Figure 5.3 to plot the deflection curve in the optical window.

Equations (5.12) and (5.13) are the deflection equation which correspond to a simply support beam with a concentrated load at the center. The force used to compute the deflection was the approximated force predicted in the previous section. The graph in Figure 5.8 demonstrates the deflection within the optical window. As discussed earlier, the maximum deflection exists at the center of the optical window.

$$
\begin{gathered}
z_{d}=\frac{F_{g} x}{48 E I}\left(3 l^{2}-4 x^{2}\right) \quad \text { For } 0 \leq \mathrm{x} \leq \frac{l}{2} \\
z_{d}=\frac{F_{g}(l-x)}{48 E I}\left(3(l-x)^{2}-4 x^{2} \quad \text { For } \quad \frac{l}{2} \leq \mathrm{x} \leq l\right.
\end{gathered}
$$

The process to approximate the fringe gradient is by using the deflection curve. A two-step process is done to determine the additional fringe gradient corresponding to the deflection curve. Firstly, rearranging equation (5.9), solving the additional fringe caused by the deflection was done along the entire diameter at the center of the optical window. Lastly, the derivative of the change in the fringe along the center of the optical window is plotted, which represent the fringe gradient due to the deflection as show in Figure 5.8. As indicated in the figure, the scanned locations used for the measurement process are taken at $0.0215 \mathrm{~m}$ and $0.0285 \mathrm{~m}$. Thus, the fringe gradient due to deflection within the location of the measured data are \pm 6 fringe/m as indicated in Figure 5.8.

The calculated fringe gradient corresponding to the deflection demonstrates a resemblance with the experimental data of the measured fringe gradient. In the measured fringes gradient, the upper fluid domain was seen to be slightly smaller than the bottom fluid domain. In similarity, the fringe gradient above the barrier has a positive shift, and the opposite below the barrier. Thus, this fringe 
gradient error caused by the deflection can be used to correct the experimental method to reduce the measurement error.

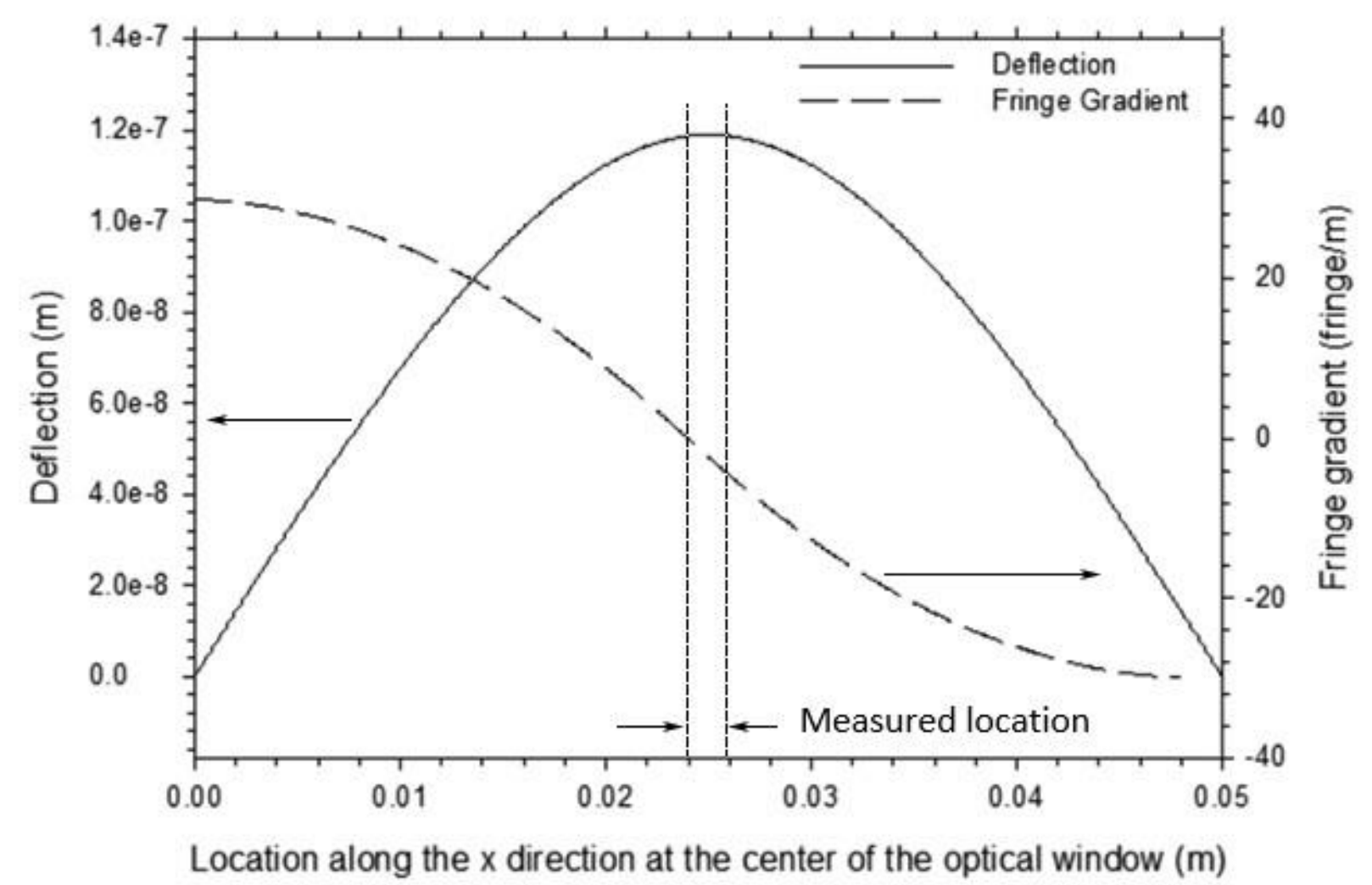

Figure 5.8: Line graph that demonstrates the number of fringes and fringe gradient caused by the deflection on the optical window.

\subsubsection{Comparison of simply supported beam solution to 3D FEA simulation}

Given the success of the highly simplified modelling (simply supported beam), a more accurate estimate was made using the finite element analysis. A three-dimensional stress analysis was simulated on SolidWorks.

The dimensions and materials of the optical window were replicated on SolidWorks. A uniform load was applied at the center of the optical window, i.e., at the location along the center of the Oring. The perimeter of the optical window on the side opposite the uniform load was simply supported. The optical window was discretized into 26,300 tetrahedral finite elements. The insensitivity of the results to the mesh density was confirmed. 
The force required to produce the maximum deflection at the center of the window of $z_{d}=$ $1.372 \times 10^{-7} \mathrm{~m}$ was calculated iteratively. The uniform force was adjusted until the desired center deflection was obtained. This was a simple iterative process since the elastic deflection is linearly proportional to the force.

Figure 5.9 shows the deflection of the optical window computed using SolidWorks Simulation. The force required to achieve the measured deflection $\left(z_{d}=1.372 \times 10^{-7} \mathrm{~m}\right)$ was found to be equal to $4.44 \mathrm{~N}$. This value compares well with the compression figure (Figure 5.7) given by the manufacturer. As previously discussed, a compression force of approximately 1-5 $\mathrm{N}$ is required to achieve a " $10 \%$ squeeze". This advanced stress analysis provides additional evidence that the magnitude of the compressive force between the optical window and O-ring sufficient to produce the measured maximum deflection.

Based on the study performed, bending of the optical window seems to be a weakness in the current design. An obvious solution to this problem is to increase the thickness of the optical window (w). Using the simply supported beam model as a guide, increasing the thickness by a factor of two will increases the second moment of area and hence, the maximum deflection by a factor of eight. However, additional sources of stress, such as thermal stresses (when the model is heated) and uneven forces applied to the four fasteners, contribute to window deflection. Thus, increasing the thickness to $30 \mathrm{~mm}$ may be required to resolve the deflection in the optical window.

The possibility of quickly replacing both optical windows with thicker versions was investigated. Unfortunately, it was found that commercially available optical windows with a diameter of 50 $\mathrm{mm}$ are only available at a thickness of $10 \mathrm{~mm}$ or less, e.g. Newport, Meller Optics, Esco optics, and Edmund optics. In the next iteration of the current experimental model, thicker optical windows will need to be ordered as a custom item. The time period for the delivery of such precision optical component (refer to Appendix $\mathrm{H}$ ) is typically two months. In addition, the Delrin frame of the current experimental model will require additional machining to accommodate the thicker optical windows 


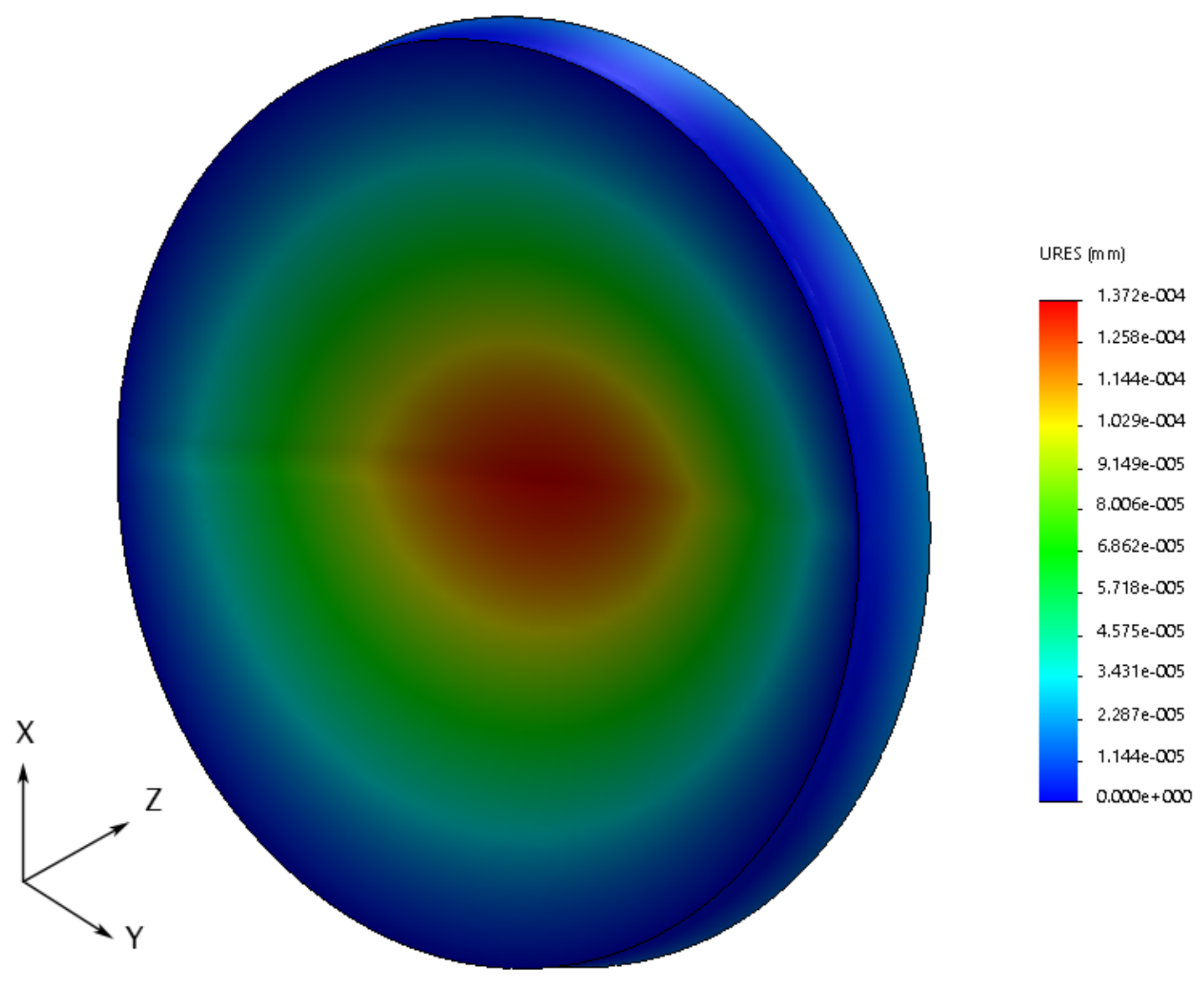

Figure 5.9: Optical window deflection simulation analysis performed on SolidWorks.

Given the restricted timelines of the current project, it was decided to proceed with the existing optical windows and attempt to correct for the stress-induced fringe distortions. As will be shown in the following sections, this approach was partly successful.

\subsection{Water - water analysis}

In this section, a proposed technique to correct for the stress-induced bending of the windows is presented, which is a subtraction technique. This technique was applied to make measurements with deionized water. A set of experiments were initially performed solely with deionized water 
in both fluid domains in order to identify the proposed technique's capability and limitations. With water in both domains the conductivity ratio is known: $\mathrm{k}_{\mathrm{n}} / \mathrm{k}_{\mathrm{w}}=1.0$.

In addition, controllable parameters which impact the measurement uncertainty of the proposed technique will be discussed, which are the temperature gradient and the fringe spacing. These preliminary experiments with deionized water provided insight into the measurement method's capabilities and limitations.

\subsubsection{The subtraction method}

With both fluid domains containing water, it was possible to use either finite or infinite fringe modes to make measurements. When making measurements in finite fringe mode, the fringes in the unheated model were set horizontal to the barrier. The image of this fringe field in the unheated model is considered a reference state. This reference fringe field is caused by two effects: (i) the non-parallelism of the test and reference beams at the second beam splitter, and (ii) the stressinduced deflection of the optical windows. When the model is heated, additional fringes are produced due to the changes in refractive index of the test fluid produced by the temperature field. These fringes are superimposed upon the reference state. Thus, by subtracting the fringe gradient in the unheated state from the fringe gradient in the heated state yields the fringe gradient solely due to the temperature field. This result can be used to calculate the temperature gradient above and below the barrier. For example, in the water-water experiments, a $10^{\circ} \mathrm{C}$ temperature difference results in a doubling of the number of fringes in both fluid domains from the unheated to heated states.

For experiments performed with water in both fluid domains, the heated model will have a greater fringe gradient than the unheated model. Thus, subtracting the fringe gradient of the unheated

model $\left(\frac{d \varepsilon}{d x}_{\text {unheated }}\right)$ from the fringe gradient of the heated model $\left(\frac{d \varepsilon}{d x}_{\text {heated }}\right)$ yields the fringe gradient at the barrier due to the temperature field $\left(\frac{d \varepsilon}{d x_{T}}\right)$. Equation (5.14) illustrates the subtraction equation for experiments performed with a base fluid and test fluid in finite fringe setting mode:

$$
\frac{d \varepsilon}{d x_{T}}={\frac{d \varepsilon}{d x_{\text {heated }}}}-{\frac{d \varepsilon}{d x_{\text {unheated }}}}
$$


With both fluid domains containing water, it was also possible to perform experiments in infinite fringe mode. In this mode, the test and reference beams are nearly parallel, and the fringe field is entirely due to stress-induced deflection of the optical windows (and other optical imperfections). In the infinite fringe mode, the fringe spacing in the unheated reference state (see Fig. 5.3) was too wide to be measured with Matlab scanning algorithm. Instead, the correction due to the deflection $\left(\frac{d \varepsilon}{d x_{S_{c}}}\right)$ was estimated based on the analysis performed in Section 5.4.2. Thus, equation (5.15) was used for experiments performed with water in both fluid domains in the infinite fringe setting:

$$
\frac{d \varepsilon}{d x_{T}}=\frac{d \varepsilon}{d x_{\text {heated }}}-{\frac{d \varepsilon}{d x_{S_{C}}}}
$$

The computed fringe gradient of both fluid domains is required to compute the relative thermal conductivity. This is calculated using equation (5.16), discussed in Chapter 2. Equation (5.14) is used to measure the relative thermal conductivity of nanofluids

$$
\frac{k_{t f}}{k_{b f}}=\frac{\left(\left.\frac{d \varepsilon}{d x}\right|_{t f}\right)\left(\frac{d n}{d T_{b f}}\right)}{\left(\left.\frac{d \varepsilon}{d x}\right|_{b f}\right)\left(\frac{d n}{d T_{t f}}\right)}
$$

Experiments performed with water in both fluid domains will have the temperature coefficient of refractive index to be the same in both domains. So, the conductivity ratio is equal to the ratio of the fringe gradients. Note that the fringe gradients in Equations (5.16) are only due to the temperature field, i.e computed using the subtraction technique (Equations (5.14) and (5.15)).

The absolute thermal conductivity can be easily measured by multiplying the thermal conductivity ratio by the thermal conductivity of water at the barrier temperature, or the thermal conductivity of the fluid in the top fluid domain:

$$
k_{t f}=\frac{\left(k_{w}\right)\left(\left.\frac{d \varepsilon}{d x}\right|_{t f}\right)\left(\frac{d n}{d T}{ }_{b f}\right)}{\left(\left.\frac{d \varepsilon}{d x}\right|_{b f}\right)\left(\frac{d n}{d T} t f\right)}
$$

The measured fringe gradient equation was developed to be suitable for the proposed technique when working with water, and the next step is to understand the measurement uncertainty associated with the technique. 
The measurement uncertainty for the proposed technique is important to be discussed as it highlights the accuracy of the documented results of the measured data. There are bias measurements with equipment used in the apparatus, along with measurement uncertainty associated with the measuring technique or scanning algorithm. A breakdown of the measurement uncertainty of the relative thermal conductivity of test fluid, and the Rayleigh number is available in Appendix F.

The method of Kline and McClintock [1953] was used to compute the measurement uncertainty. The uncertainty will be difference for the measurements performed with deionized water compared to those with nanofluids. The thermal coefficient of refractive index can be eliminated in the equation, which means the measurement uncertainty is solely dependent on the measured fringe gradient from both fluid domains. For this reason, the measurement uncertainty for water-water experiments will be lower than for nanofluids.

\subsubsection{Finite fringe spacing and temperature difference}

In each experiment there were two adjustable parameters which influenced the measurement uncertainty of the proposed technique. The number of horizontal fringes in the unheated reference state can be set arbitrarily by adjusting the angle of the test and reference beams. Also, the number of fringes produced by the temperature field can be adjusted by changing the temperature difference between the hot and cold plates. In this section, the effect of these parameters are investigated (for the water-water case) in order to find reasonable settings that minimize the experimental uncertainty. These settings were then used for the investigation performed with the nanofluid.

\section{Temperature difference}

An appropriate working temperature difference is required to produce consistent results. With interferometry, there a limitation on the number of fringes can be used within the fluid domain. When the fringe spacing is extremely small, the fringes at surface becomes difficult to distinguish, in part due to refraction effects. Also, the number of image pixels from fringe peak to fringe peak 
becomes small. Figure 5.10 shows sample images in the unheated and heated states at temperature difference of $6^{\circ} \mathrm{C}$. This represents the approximate minimum temperature difference that can be used. At temperature differences less than $6^{\circ} \mathrm{C}$, the difference in the fringe gradients in the unheated and heated states becomes too small to accurately measure. Figure 5.11 shows these unheated and heated condition for a temperature difference of $10^{\circ} \mathrm{C}$. These conditions were found to be near the ideal temperature difference suitable for the subtraction technique. It represents a balance between having fringes that at too fine at the surface while having a significant difference between the unheated and heated images.

a)

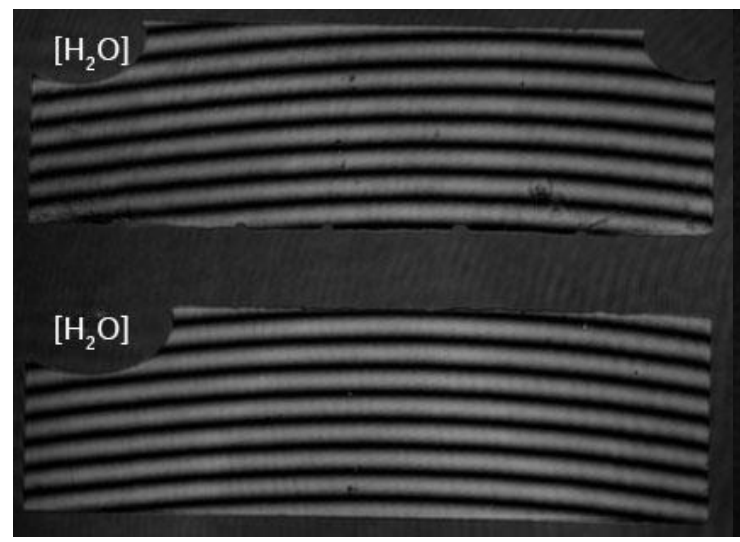

b)

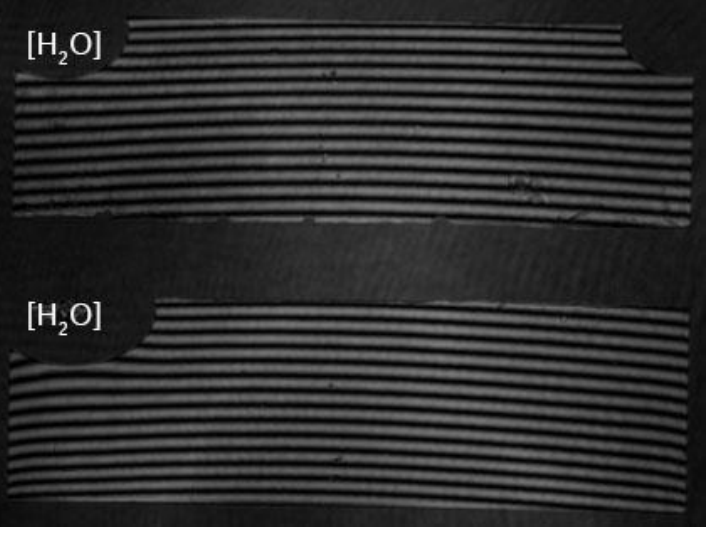

Figure 5.10: Finite fringe setting with $6^{\circ} \mathrm{C}$ temperature difference, a) unheated model, b) heated model.

a)

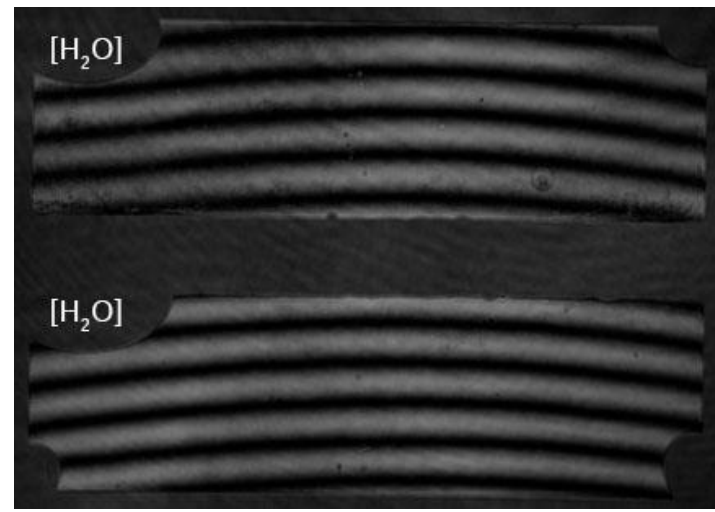

b)

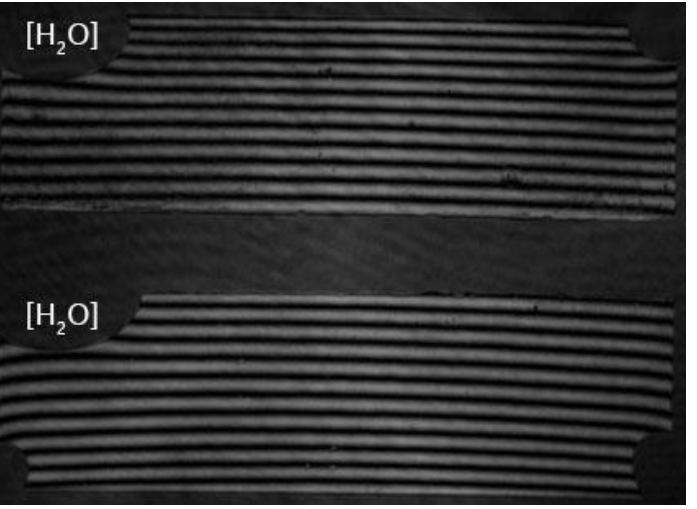

Figure 5.11: Finite fringe setting with $10^{\circ} \mathrm{C}$ temperature gradient, a) unheated model, b) heated model. 
To the unaided eye, the captured images in both unheated and heated state for both temperature differences may appear similar, however, the uncertainty of the extracted fringe gradient was different. Consistently the experiments performed with a $10^{\circ} \mathrm{C}$ temperature difference have shown a lower uncertainty than with the experiments performed with $6{ }^{\circ} \mathrm{C}$ temperature difference. Thus, all experiments reported for the proposed technique will be done using a total temperature difference of $10^{\circ} \mathrm{C}$.

\section{Fringe spacing}

The unheated finite fringe spacing is an important setting that affects the measurement uncertainty for the proposed technique. The entire process of performing the image processing technique is dependent on the reference fringe spacing. It was found that increase the number of fringes in the reference image reduced the scatter in the calculated fringe gradient using the subtraction technique. This was found to dramatically improve the measurement uncertainty. Through trial and error experimentation, a suitable number of fringes in the unheated reference image was found to be approximate 8 in each fluid domain (as in Figure 5.12a).

\subsubsection{Experimental results}

\section{Finite fringe setting results}

The measurements performed with deionized water in both fluid domains in finite fringe setting mode will be discussed, which includes the measured absolute and relative thermal conductivity and the uncertainty of the measurements. The data obtained with deionized water were performed with the barrier temperature at $20^{\circ} \mathrm{C}$, which was achieved by setting $\mathrm{T}_{\mathrm{H}}$ to $25^{\circ} \mathrm{C}$ and $\mathrm{T}_{\mathrm{C}}$ to $15^{\circ} \mathrm{C}$. Figure 5.12 show the model's unheated and heated states. Notice that the uniform fringe spacing in the heated condition illustrates that the temperature field is conduction dominated. As predicted numerically (in Chapter 4) buoyancy-induced convective flow is weak.

Figure 5.13 shows fringe gradient near barrier due to the temperature field in both the upper and lower fluid domains. These results correspond to the interferograms shown in Figures 5.12a and 
5.12b. This figure presents 10 sets of measurements (scans) on each side of the barrier, near the center line of the model. The scatter in the data is caused by random variations in the fringe spacing mainly due to optical imperfections. A key feature of experiments performed with water is that the results are independent of the temperature coefficient of refractive index. By working with the same fluid in both fluid domains, the ratio of temperature coefficient of refractive index is eliminated, resulting in the thermal conductivity ratio being equal to the ratio of the measured fringe gradients. Since both fluid domains contain water, the fringe gradients should be equal on both sides of the barrier. In Figure 5.13, the difference in the mean fringe gradients on the hot and cold side of the barrier is $2.8 \%$ due to measurement errors.

a)

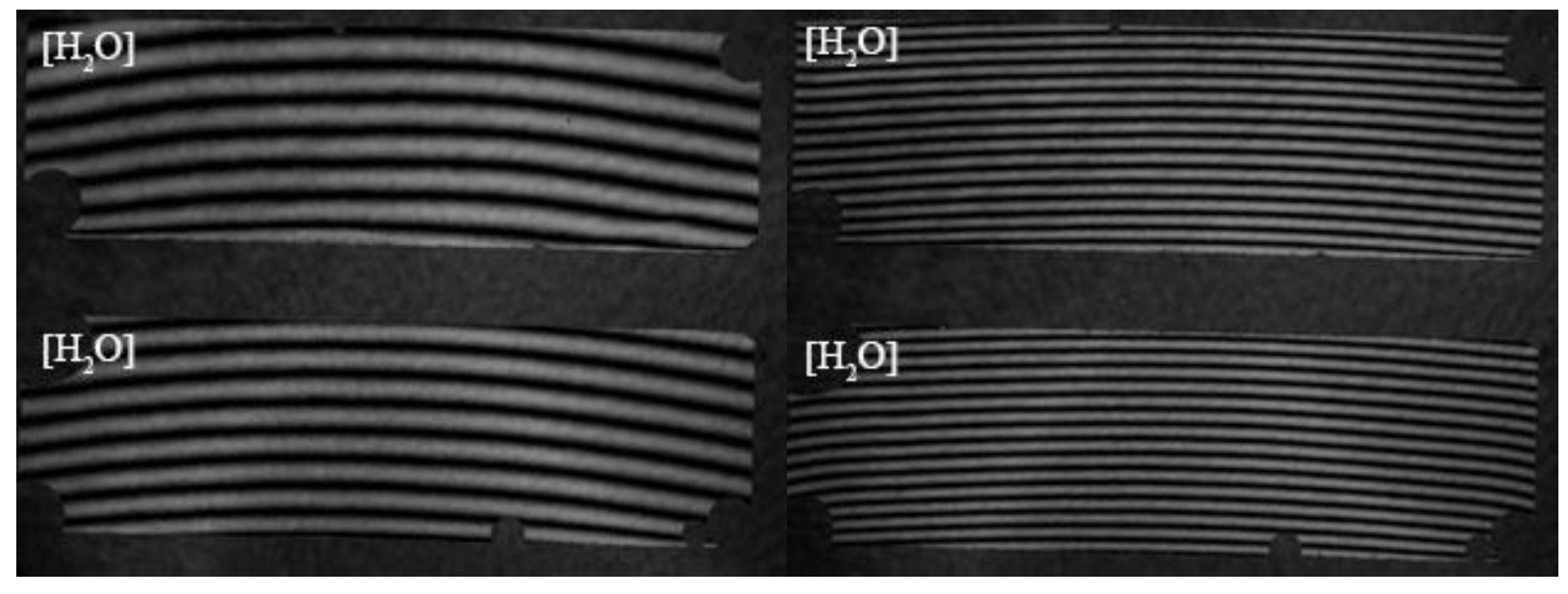

Figure 5.12: Experiment performed in finite fringe mode with deionized water in both fluid domains at a temperature difference of $10^{\circ} \mathrm{C}$ with the barrier temperature set at $20^{\circ} \mathrm{C}$, a) unheated model, b) heated model. 


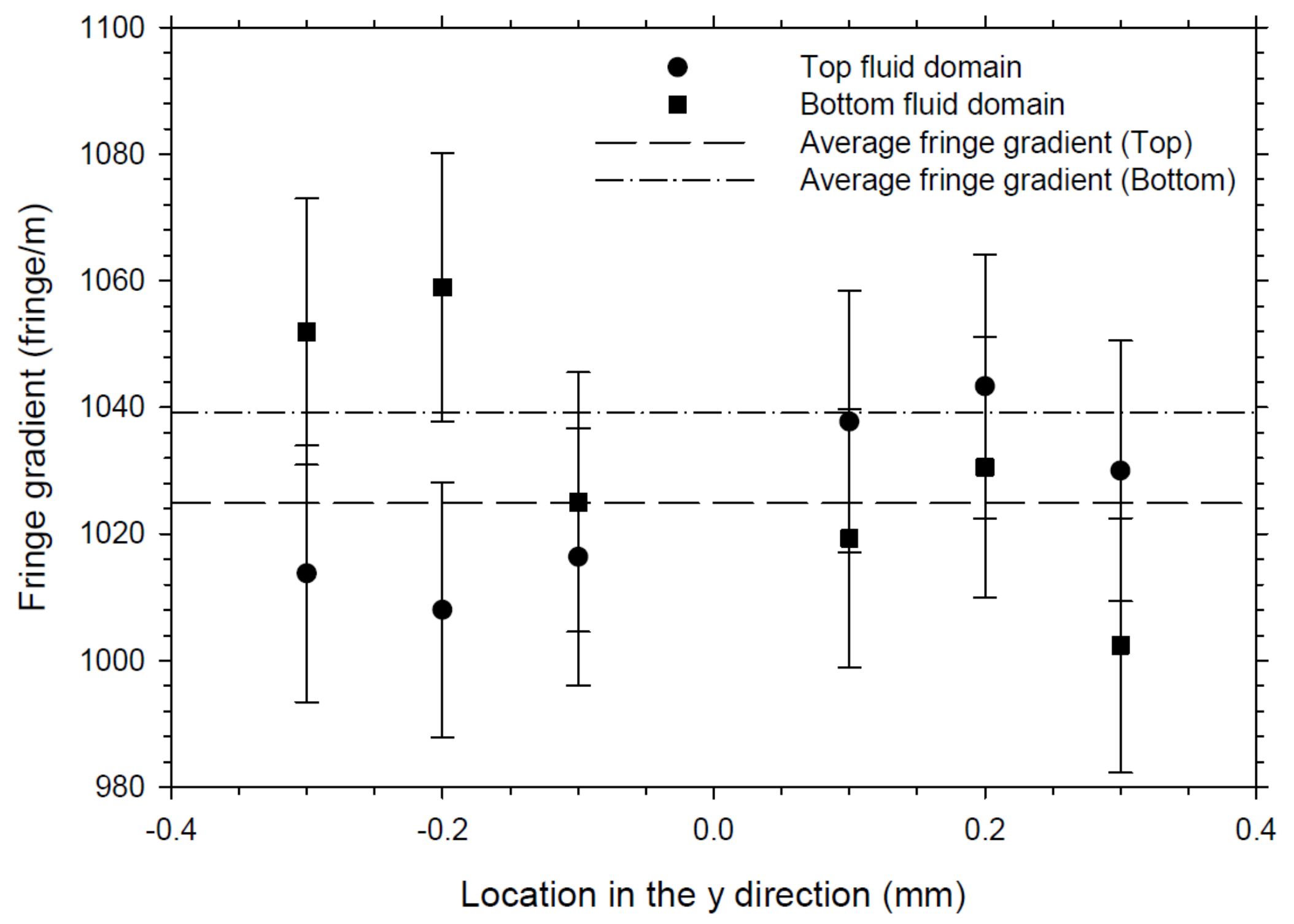

Figure 5.13: Fringe gradient due to the temperature field at the barrier, extracted from the images shown in Figure 5.12. 


\section{Infinite fringe setting results}

Measurements for the water-water case were also made using the infinite fringe setting, for similar conditions that were used with finite fringe mode experiments. The images seen in Figure 5.14 are of the model's unheated and heated state which were taken in infinite fringe mode.

a)

\section{$\left[\mathrm{H}_{2} \mathrm{O}\right]$}

$\left[\mathrm{H}_{2} \mathrm{O}\right]$ b)

\section{$\left[\mathrm{H}_{2} \mathrm{O}\right]$}

10

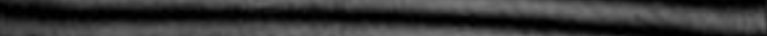

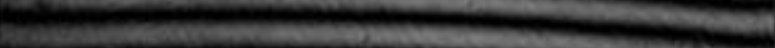

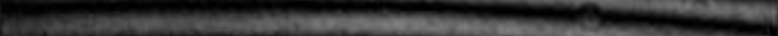

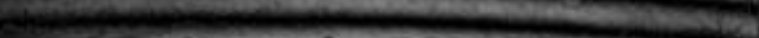

$\left[\mathrm{H}_{2} \mathrm{O}\right]$

1

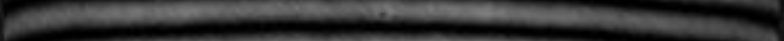

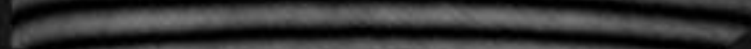

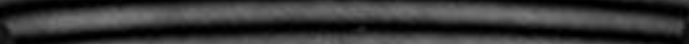

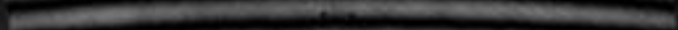

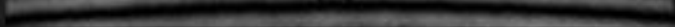

Figure 5.14: Experiment performed in infinite fringe mode with deionized water in both fluid domains at a temperature difference of $10^{\circ} \mathrm{C}$ with the barrier temperature set at $20^{\circ} \mathrm{C}$, a) unheated mode, b) heated model.

The fringe gradient can be seen to be uniform, symmetrical at the surface, and consistent in terms of additional fringes due to deflection in the optical window. By observing the unheated model, roughly half of fringe can be seen to exist in each fluid domain. This stress-induced fringe gradient was used to correct the results, as discussed in Section 5.2. The fringe gradient on both sides of the barrier due to the temperature field is shown in Figure 5.15. These results were extracted from the Figure 5.14. Again, in an ideal error-free experiment, the fringe gradients would be identical on both side of the barrier. In the current experiment, the average difference in fringe gradients on the hot and cold sides of the barrier was $6.89 \%$. This error is higher than the experiment done under similar conditions in finite fringe mode. 


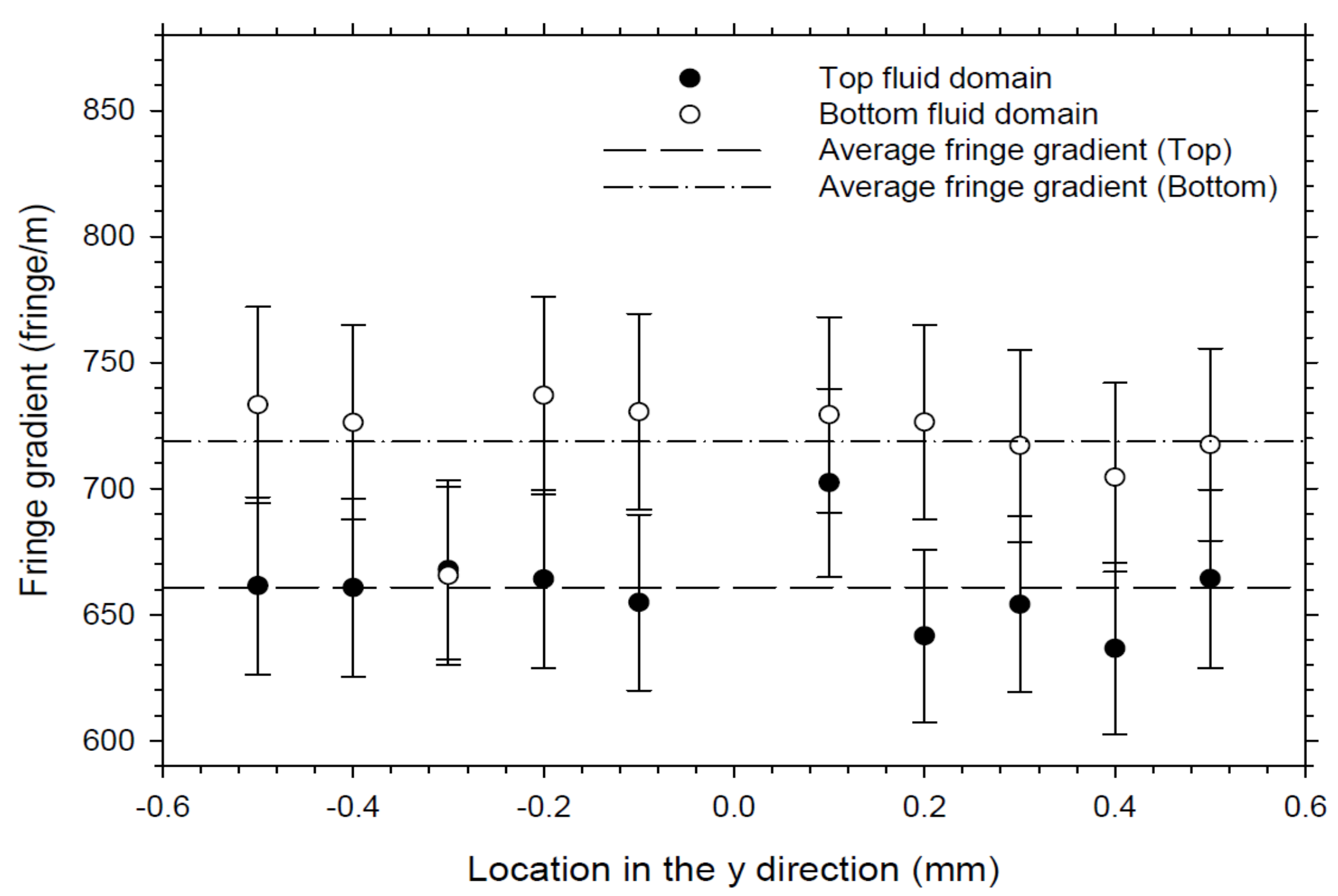

Figure 5.15: Fringe gradient at the barrier due to temperature, measured with water in both fluid domains in infinite setting mode shown in Figure 5.14. 
The experiments done in finite and infinite fringe modes yield a conductivity ratio. The conductivity of the test fluid in the lower domain is determined relative to the conductivity of the fluid in the upper domain (the base fluid). In the current experiments, both fluids were deionized water. To illustrate the experimental method, the conductivity of the test fluid in the lower domain has been calculated using Touloukian's data [1970] of the absolute thermal conductivity of deionized water (the test fluid). Figure 5.16 shows a comparison of the experimental results obtained using finite and infinite fringe modes. The comparison between the two setting modes demonstrates that the technique was successful in measuring the thermal conductivity of water, with a level of uncertainty that is typical for this type of optical method [Roeleveld, 2013].

An important fact that is required to be addressed is the difference between the measurement uncertainty of the two fringe settings, which indicates the approximation used in infinite setting might not be as accurate as intended. With a temperature difference of $10^{\circ} \mathrm{C}$ when the model was heated, the thermal expansion of the optical window should impact the deflection. It might be difficult to notice from observing the images, but the first few fringes near the barrier are likely greater in fringe spacing due to the thermal expansion impacting the deflection. However, the thermal expansion of the optical window was not considered as part of the fringe gradient correction due to deflection analysis as discussed in Section 5.2.2. Although the unmeasurable forces from the fastener make it difficult to predict the change in the deflection considering the thermal expansion of the optical window. In other words, the difference in the measure uncertainty highlight the important in replacing the optical window to improve the performance of the measuring technique.

It should be noted that the results presented in Figure 5.16 are from a single experiment, with images taken in finite and infinite fringe modes. Ideally, these results should be repeated to give an indication of the reproducibility of the method. Unfortunately, reproducibility experiments were found to be challenging, due to the difficulty in achieving a low stress state in the optical windows. In the experiment shown in Figure 5.14a, it can be seen that there are a low number of fringes produced by the stress in the optical windows. Thus, the correction was small. Such a low stress state was difficult to replicate. This issue can be resolved by using thicker optical windows. 


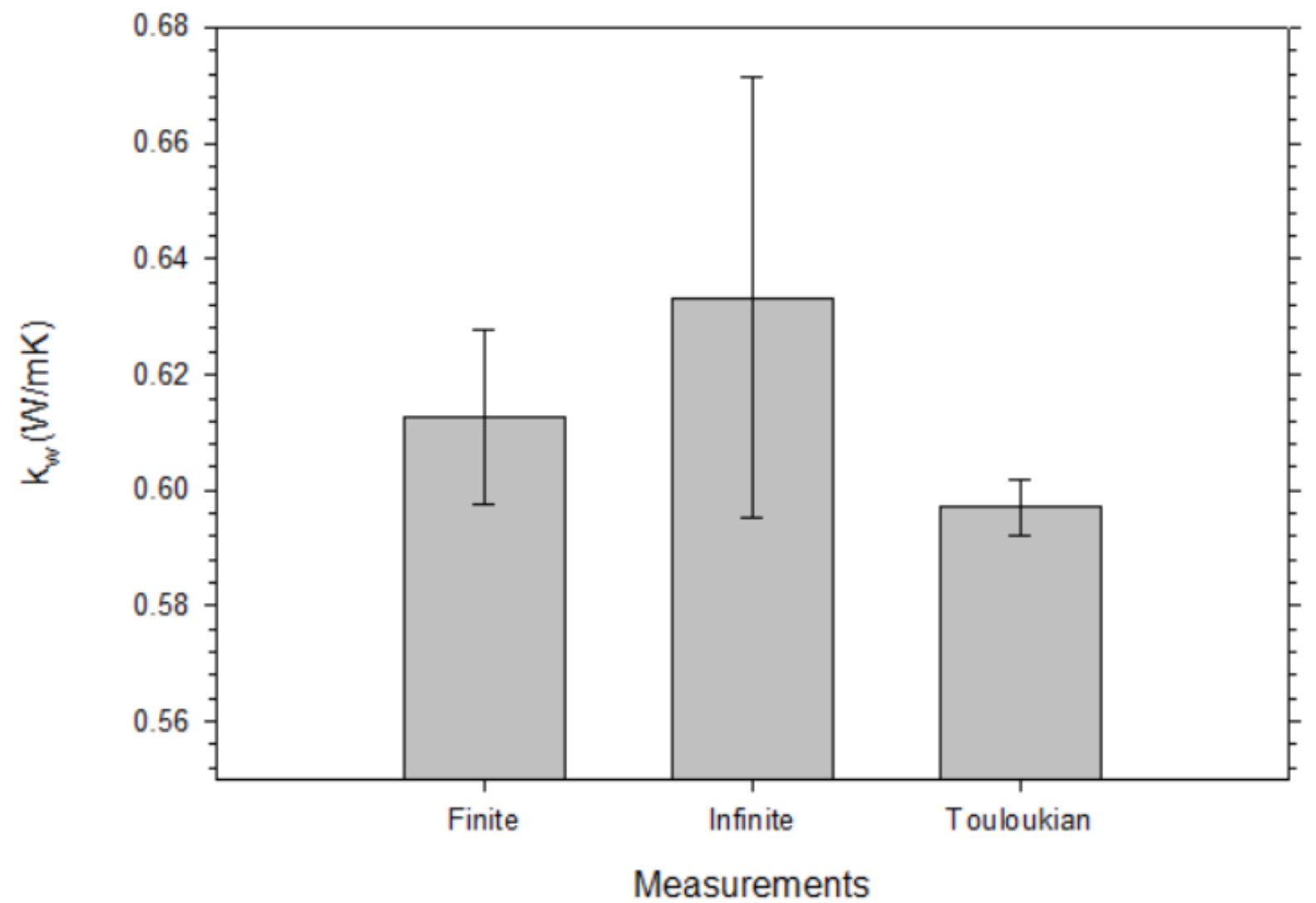

Figure 5.16: Absolute thermal conductivity of water at $20^{\circ} \mathrm{C}$ measured using finite and infinite fringe settings.

\subsection{Water - nanofluid analysis}

Despite the difficulties with limiting the stress-induced fringes, two experiments were conducted with $\mathrm{SiO}_{2}$-water nanofluid. Even if unsuccessful, it was of interest to confirm the quality of the fringe field in the nanofluid. As discussed in Chapter 1, Yousefi et al. [2015] have reported that laser interferometry can be done with $\mathrm{SiO}_{2}$-water nanofluid up to a concentration of $20 \%$ by volume. In the current work, experiments were done for $\mathrm{SiO}_{2}$-water nanofluid at concentrations of $7.3 \%$ and $10.1 \%$ by volume. 


\subsubsection{Leakage issues}

Figure 5.17 is a shadow graph of the unheated model with deionized water in the upper domain and $10 \% \mathrm{SiO}_{2}$-water nanofluid (by volume) in the lower domain. This image does not have any fringes present, because the picture was captured before the light passes through the second beam splitters (BS2, Figure 2.5). Note that quality of the image are somewhat poor because this was an unfocused image taken with a cell phone camera. What appears to be happening in Figure 5.17 is that nanofluid and water are seeping through the barrier and exchanging places. Concentration gradients due to the leakage across the barrier produces distortions due to refraction effects. Of course, such leakage would not have been visible for the water-water experiments because there would be no concentration gradient. This image highlights the difficulty of achieving a near perfect seal between the two fluid domains, while not overstressing the optical windows. Again, this may be resolved by using thicker optical windows, which will allow the fasteners to be fully tightened.

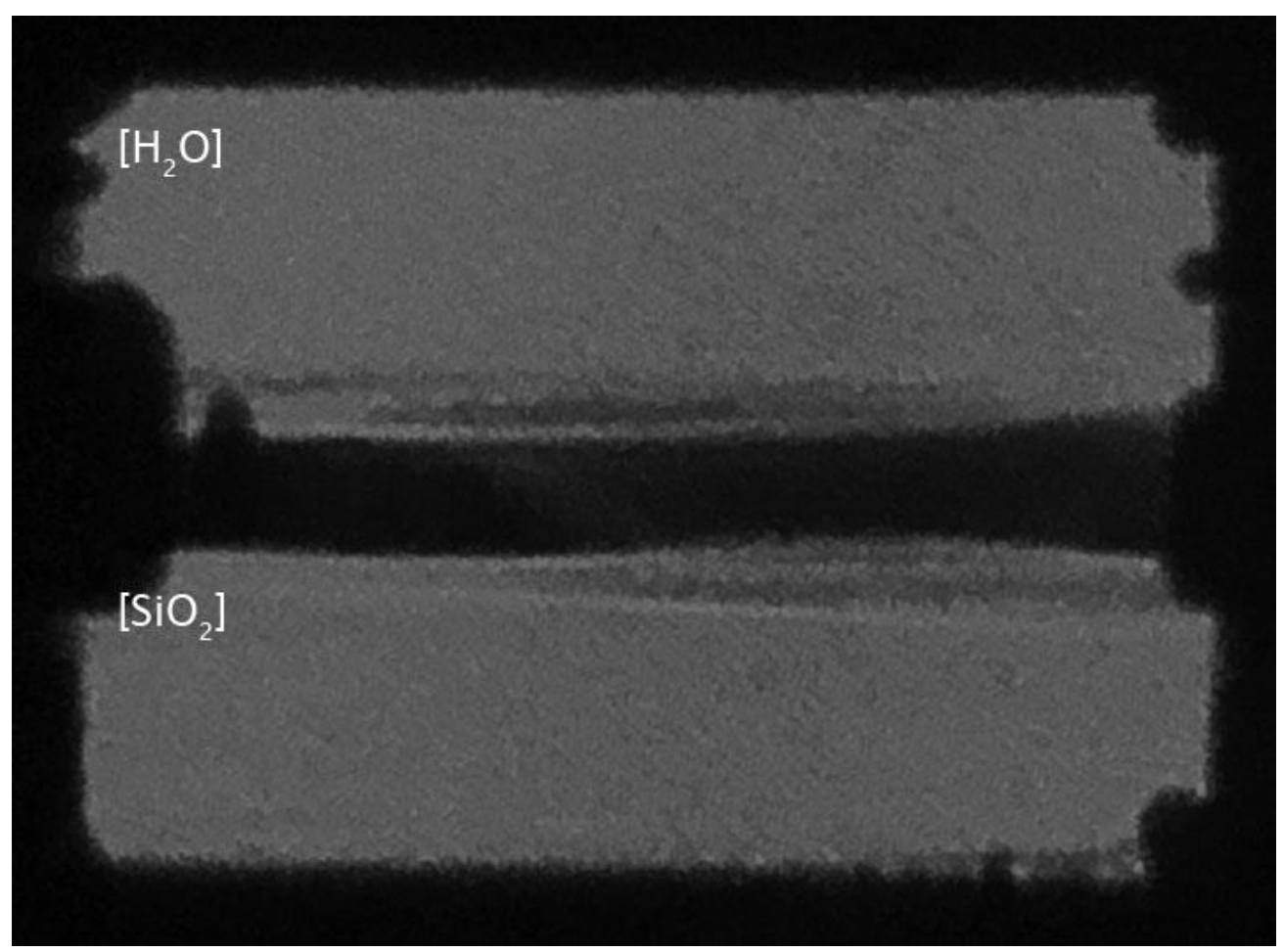

Figure 5.17: Shadowgraph images of the unheated model with deionized water in the upper domain and $10 \% \mathrm{SiO}_{2}$-water nanofluid (by volume) in the lower domain. 
The second issue was the expansion of the nanofluid when the model was heated. It is expected the fluid in the model would expand and increase the pressure within the domain when subjected to a temperature gradient. In fact, the air bubbles seen within the fluid domain are helpful in terms of allowing the fluid to expand and reduce the pressure build up, as the air bubbles reduce in size. However, even with the air bubbles present in the nanofluid domain, the pressure within the fluid domain increased sufficiently to promote leakage of the nanofluid into the upper fluid domain. The thermal expansion of the nanofluid at varying concentration is an unclear area, and the numerous failed experiments have shown that the thermal expansion for concentrations of more than $10 \%$ by volume concentration within a $5^{\circ} \mathrm{C}$ temperature gradient was an issue for the model.

\subsubsection{Nanofluid results and comparison with data from the literature}

The relative thermal conductivity of $\mathrm{SiO}_{2}$-water nanofluid at $7.3 \%$ and $10.1 \%$ by volume concentration were measured using the subtraction technique. Figure 5.18 shows interferograms of the unheated and heated model with a test fluid of $\mathrm{SiO}_{2}$-water nanofluid with $10.1 \%$ by volume. Note that due to the leakage issues experienced with the experiment, the fasteners were required to be tightened significantly more than for the experiments performed with water. As a result, the deflection in the optical windows have drastically increased. This is evident from the very large difference in the finite fringe spacing in the two fluid domains for the unheated condition. At this level of stress, thermal expansion effects become a concern. When the model is heated, it is likely that the state of stress in the optical windows will change. If this happens, then subtraction of the unheated reference image will no longer be a reliable correction.

Figure 5.19 shows the relative thermal conductivity of the two nanofluids, measured using the subtraction method. The measured values are also compared with correlations from the literature. It can be seen the measured conductivity ratios are much higher than predicted by published correlations. As discussed above, because of the high state of stress the optical windows the current experimental technique does not give reliable results. However, it can be seen in Figure 5.18 that the quality of the fringes is high. After modification of the model to accept thicker optical windows, it should be possible to make measurements with these concentrations of $\mathrm{SiO}_{2}$-water nanofluids. 
a)

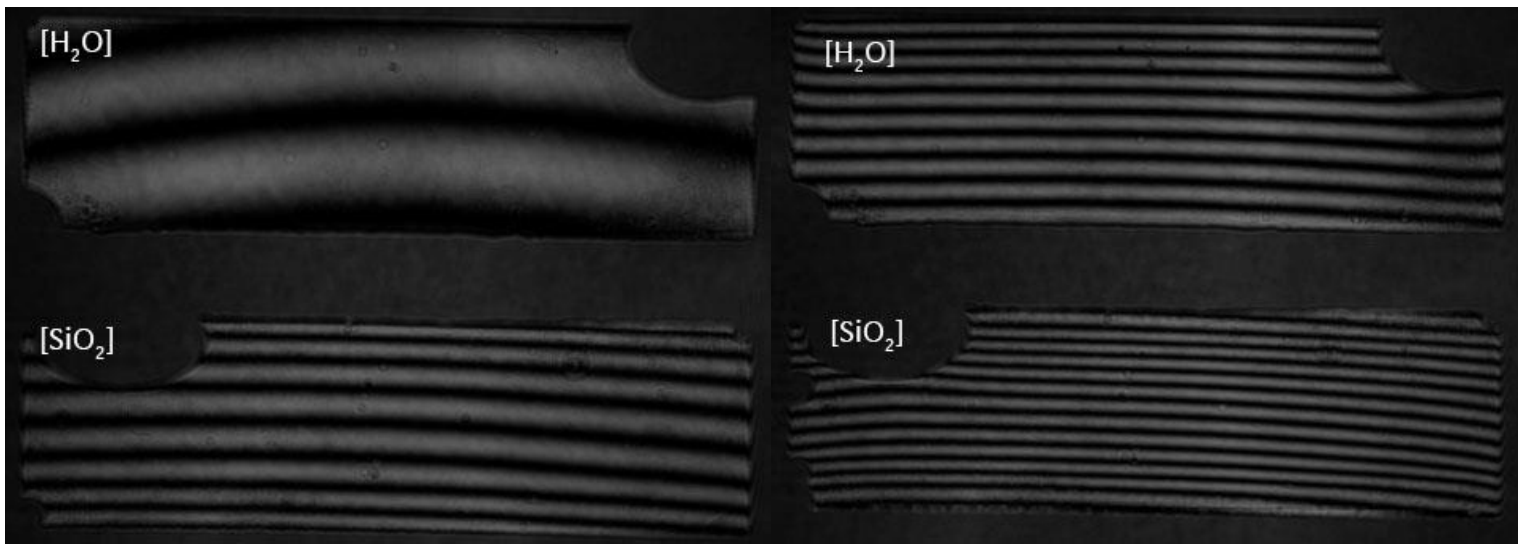

Figure 5.18: Experiment performed with $\mathrm{SiO}_{2}$-water at $10.1 \%$ by volume concentration with a temperature gradient at $10^{\circ} \mathrm{C}$, a) unheated model, b) heated model.

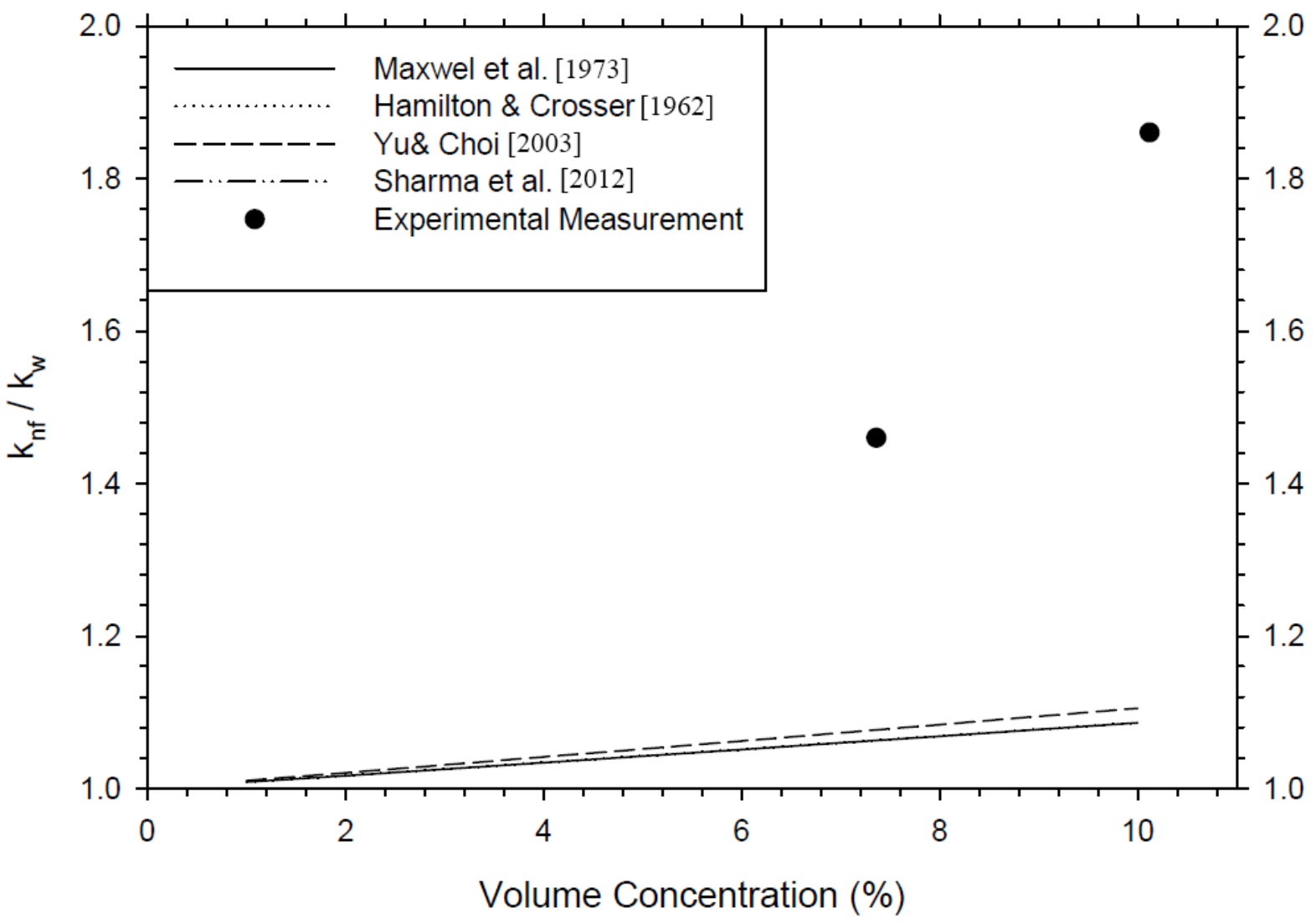

Figure 5.19: Comparing the relative thermal conductivity measurements performed with $\mathrm{SiO}_{2}-$ water at $20^{\circ} \mathrm{C}$ in finite fringe setting mode to correlations discussed in the literature review. 


\section{Chapter 6: Conclusions and Recommendations}

In this chapter, the main conclusions obtained from the study will be presented. Also, the recommendations for future work will be discussed.

\subsection{Conclusions}

This research considered the development of a comparative optical method to measure the thermal conductivity of a test fluid relative to that of a base fluid of known conductivity. Although the method could be applied to any transparent fluid, the motivation for this work was the desire to measure the thermal conductivity of nanofluids. The method was based on laser interferometry, using a classical Mach-Zehnder interferometry (MZI) optical setup. The method is based on onedimensional heat transfer across a high conductivity barrier that separates the two fluids. A test model was designed in CAD software and the performance was evaluated using computational fluid dynamics (CFD). Based on these predictions, an experimental model was constructed for use in the MZI. Preliminary experiments were performed with deionized water as both the test fluid and base fluid. Subsequently, experiments were performed with a $10 \% \mathrm{SiO}_{2}$-water nanofluid as the test fluid.

This research demonstrates that the technique to measure the thermal conductivity of nanofluids non-intrusively by using an interferometer was a partial success. The numerical analysis using CFD has shown that the technique is viable. The results of a linear temperature profiles in the fluid domains at the surfaces of the aluminum barrier indicates that the technique to measure the thermal conductivity is consistent with the one-dimensional assumption. The study confirmed that the temperature fields near the barrier surfaces in both fluid domains is conduction dominated and thermally stratified. As well, the numerical investigation confirms the design was suitable for the proposed technique, in terms of performing experiments with the interferometer.

As a result, one of the main contributions of the research carried out in this study is the design and implementation of the technique, and the ability to measure the thermal conductivity of the test fluid. The experiment demonstrates the method can measure the thermal conductivity of both water 
and $\mathrm{SiO}_{2}$-water nanofluid at $10 \%$ by volume concentration. As well as identifying a few technical issues associated with the model, the results demonstrate the accuracy of the measuring technique by comparing the measured data for deionized water to the thermal conductivity data of Touloukian et al. [1970]. Also, an investigation comparing the measured data of $\mathrm{SiO}_{2}-$ water to the correlation discussed in the literature review was performed. However, due to the design issues present in the experimental model that were difficult to overcome, when the test fluid was different from the base fluid, the measurements were not successful.

The main results achieved in this research project are summarized as follows:

1. Laminar steady state three-dimensional CFD analysis of the experimental model demonstrated that, at a temperature difference suitable for laser interferometry, the buoyancy-induced convection in both fluid domains was very weak. As a result, the temperature field was thermally stratified, and conduction dominated, which is desirable for this technique. Furthermore, the CFD modelling indicated that heat flux across the high-conductivity barrier was strongly one-dimensional, i.e., the heat flux from the base fluid was equal to the heat flux to the test fluid to a high level of accuracy.

2. Preliminary experiments with $\mathrm{SiO}_{2}$-water nanofluid as the test fluid and water as the base fluid revealed that there was a different fringe gradient in the two fluid domains (in the finite fringe mode) even when the model was unheated. This was a surprising result. Optical path length analysis showed that the fringe gradient difference is caused in part by the non-parallelism of the optical windows. It has been shown that there will always be a difference in the fringe gradient in the two fluid domains, even in the unheated mode, if the optical windows are not parallel and the two fluids have different indices of refraction.

3. In addition, it has been found that bending of the optical windows caused by the force of the gasket and fasteners was an addition source of the unexpected fringe gradient difference in the two fluid domains for the unheated condition. This result was demonstrated using simplified and 3D finite element stress analysis. It was found that increasing the thickness of the optical window by a factor of six will make the fringe gradient error due to the deflection negligible. However, with the current design $(t=$ 
$5 \mathrm{~mm}$ ), it was shown that bending of the windows is the dominant source of difference in the fringe gradients in the two fluid domains.

4. When experiments were done with $\mathrm{SiO}_{2}$-water nanofluid as the test fluid, there was visual evidence in the shadowgraph of the model that leakage was occurring across the barrier. It could be seen that the test fluid and base fluid were mixing. Tightening of the fasteners to reduce leakage was found to induce significant bending of the optical windows. For this reason, measurements performed with nanofluid were not successful with the current model design. However, the current research suggests that this technical issue may be resolved by using thicker optical windows.

5. Despite the lack of success in making measurements with nanofluids, it is important to note that this work demonstrates that clearly visible interference fringes can be obtained in $\mathrm{SiO}_{2}$-water nanofluid up to a concentration of $10 \%$ by volume. This result is consistent with the prior work by Yousefi et al. [2015].

\subsection{Recommendations}

\subsubsection{Introduction}

Chapter 5 has outlined the dominant errors which exist in the model and the results of the proposed technique. The technique was a partial success, but there is room for improvement. The focus of this section is to outline possible recommendations to improve the design and procedure, which could reduce the measurement uncertainty of the technique.

\subsubsection{Recommendations for improvements to the experimental model}

\section{Optical Windows}

As discussed in Chapter 5, replacing the optical windows with a thicker window could enhance the performance of the measuring technique. By changing the thickness of the optical window from $5 \mathrm{~mm}$ to $30 \mathrm{~mm}$ should overcome the deflection. The increased thickness was designed to 
accommodate the tightness of the fastener and the thermal expansion of the optical window. By driving the deflection close to zero, it should reduce the extra number of stress-induced fringes. In addition to replacing the optical windows, the Delrin optical frame must be re-machined to fit the new optical window size.

A quote from Esco optics (given in Appendix $\mathrm{H}$ ) is provided to highlight the specifications of the new optical windows, cost, and time to manufacture the optical windows. The effort to purchase new optical windows and redo the experiments was a process that could not be done due to the time required to manufacture the optical window and re-machine the components of the model. The time required to manufacture the new optical window was quoted from Esco optics, which should provide an approximate timeline to plan ahead for the future. The specification of the new optical windows will be a N-BK7 round optical glass, diameter set to $50 \mathrm{~mm} \pm 0.125 \mathrm{~mm}$, the thickness as $30 \mathrm{~mm} \pm 0.125$, the surface flatness of $\lambda / 10$, clear aperture at $90 \%$ of the radius, and the edges being fine grounded. The quote indicates that delivery would take about 7 weeks, at the cost of $\$ 3,360$ (Canadian dollars).

Once the optical windows have been replaced, the next step is to redo the experiments. The experiments with $\mathrm{SiO}_{2}$-water nanofluid up to $10 \%$ by volume concentration should be repeated and compared to the data shown in chapter 5. The ideal fringe pattern should not be the same as demonstrated in Figure 5.1, rather one or two more fringes should be present in the test fluid compared to the base fluid. By replacing the optical windows, the dominant error should be the non-parallelism windows. The results are should likely increase the number of fringes by roughly $2 \%$ in the test fluid domain compared to the base fluid. This will have increased the performance of the measurement technique and perform measurement in infinite fringe mode with nanofluid.

\section{Assembly method and flexible support}

An alternative method to secure the optical windows with the model assembled would be a step forward to improve the model's performance. In the current design, two optical frames are used to keep the optical window from moving out of position. Due to the fluctuation in tightening the fasteners to prevent leakage and align the fringes, the optical windows will never be perfectly parallel with each other as discussed in the Chapter 5. However, a solution to solve both the non- 
parallelism in the optical window and secure the optical windows position, an alternative design should be considered. This can be achieved by setting one optical window fixed in position and the second optical window flexible to be tilted in both $\mathrm{x}$ and $\mathrm{y}$ axis.

This suggested alternative design will further complicate the model but will resolve the nonparallelism windows problem. With the ability to control the angle by a very small degree, this could reduce the angle offsetting the parallel window, and prevent additional fringes. Based on the equation as shown in Chapter 5, by driving the angle to zero, the ability to set infinite fringe with different refractive index compared to water can be achieved.

\section{Model Size}

Increasing the overall size of the model is a potential opportunity, as doubling overall size should yield similar results and simplify the operation. The model was initially design achieve nearly pure conduction, with very little to no convection within the fluid domain. However, the focus of the technique was to extract measurements at the surface of both fluid domains. Thus, increasing the design size can be an option to be considered because measurements are made at the surface of the barrier that is conduction dominated.

The advantages of increasing the overall size would simplify the maintenance process. The procedure of dismantling and resembling the model between experiments was cumbersome and time consuming due to the very small size of the fasteners. For these reasons, increasing the overall size of the model would benefit the procedure and operation for the technique and could likely achieve similar results. 


\subsubsection{Recommendation for further research}

\section{Evaluate the effect of thermophoresis}

Studying the effect of thermophoresis could be a potential investigation to be considered. The current model had demonstrated the difficulty to measure the thermal conductivity of $\mathrm{SiO}_{2}$-water nanofluid due to the deflection in the optical windows. In the unheated state, a significant amount of force was required by the fasteners to prevent leakage from occurring between the two domains. It would have increased the initial deflection in the optical window and increase the number of fringes to occur within both fluid domains. When the model was heated, the deflection could have increased further, which would significantly impact the measurement, as shown in Figure 5.23. However, if the optical windows were replaced with a thicker lens, then deflection could be reduced to very little to no impact on the path length. In other words, the process to measure the thermal conductivity of $\mathrm{SiO}_{2}$-water and $\mathrm{Al}_{2} \mathrm{O}_{3}$ - water with the model would be viable, and the next step could be investigating the effect of thermophoresis.

Particle migration due to the temperature gradient could change the local concentration, and hence the local thermal conductivity. Khalili et al. [2017] have demonstrated this effect with micropipettes in a heated enclosure with free convection. A study is needed to determine if this is a significant effect in the present experiment setup.

A procedure to study the effect of thermophoresis could be done by heating the model from below. In the current arrangement, the hot plate was located on the top of the model, and the cold plate was set on the bottom. This arrangement reduced mixing in both domains because the system was thermally stratified. The local volume concentration in the test fluid domain could be different than anticipated for experiments performed with nanofluids. However, by switching the hot/cold plate, the test fluid domain will experience a small effect of mixing due to convection. By slightly increasing the natural convection in the fluid domain, this will likely maintain the homogenous mixture of the nanofluid in the model during the experiment. 


\section{Comparison with a secondary experimental technique}

Comparing the experiment results to a secondary intrusive/non-intrusive technique could improve the quality of the comparative analysis. In the current study, the experimental data was compared to the correlation used to predict the thermal conductivity. However, Sharma's correlation [2012] are not specifically designed for $\mathrm{SiO}_{2}$-water nanofluid yet was used by many researches. As mentioned in Chapter 1, there are many intrusive techniques available to measure the thermal conductivity of the fluid. A technique commonly used and available is the transient hot-wire method. The purpose of the comparison was to understand the difference in the measurements extracted by the two experimental techniques under the same conditions.

The outcome of many experimental investigations on measuring the thermal conductivity of nanofluid had varied because of the process used to prepare the nanofluid. Majority of the experimental investigation on the nanofluid's thermal conductivity is inconsistent. Either the particle size, shape, mixing technique, and the measured local volume concentration are parameters that are varying in majority of the documented results performed experimentally. Comparing the technique's experimental results to other experimental results discussed in the literature would be challenging to reach a consensus on the nanofluid's thermal conductivity.

The ability to measure the thermal conductivity of a nanofluid by two different experimental techniques under the same conditions would provide a more meaningful and better comparison. Control and consistency are critical elements to reach a proper conclusion in experimental investigations. The two experimental techniques could potentially spot a similar pattern in the results, such as common errors in the results, or evidence to support the effect of thermophoresis. Compared to the correlations that have considered a few assumptions about the nanofluid's property as discussed in Chapter 1, a comparison with a secondary experimental technique could result to a reliable assessment. 


\section{Appendix A: Water properties}

All of the properties that were used in the calculation with the proposed method were based on Thermophysical Properties Research Center (TPRC), Purdue University [Touloukian, 1970]. An extensive collection of data from various experimental techniques were gathered to compile a tabulated data set and correlation for the thermal conductivity, specific heat, thermal diffusivity, and viscosity.

\section{A.1 Thermal conductivity}

A set of recommended values were provided by Touloukian [1970], that were used to determine the uncertainty of the collected data and interpolate the properties based on the temperature operated for the experiment. Table A.1 demonstrates the thermal conductivity corresponding to the temperature within a measurement uncertainty of $1 \%$.

Table A.1: Thermal conductivity of water at varying temperatures.

\begin{tabular}{|c|c|}
\hline Temperature, $\mathbf{T}(\mathbf{K})$ & $\begin{array}{c}\text { Thermal Conductivity } \mathbf{k}, \\
\mathbf{W} / \mathbf{m} \cdot \mathbf{K}\end{array}$ \\
\hline 260 & 0.5388 \\
\hline 265 & 0.5470 \\
\hline 270 & 0.5551 \\
\hline 280 & 0.5818 \\
\hline 290 & 0.5918 \\
\hline 300 & 0.6084 \\
\hline 310 & 0.6233 \\
\hline 320 & 0.6367 \\
\hline 330 & 0.6485 \\
\hline
\end{tabular}




\section{A.2 Specific heat}

There were 20 sources that were used to gather the data on the specific heat of water, and a correlation was available for particular ranges of temperature. For the interest of the project, the correlation of the temperature between $-0.15^{\circ} \mathrm{C}$ and $136.85^{\circ} \mathrm{C}$ is provided by equations (A.1). Where the temperature in equation (A.1) is in K. Table A.2 shows the specific heat of water at varying temperatures.

$$
\begin{gathered}
c_{p}=\left(2.13974-\left(9.68137 \times 10^{-3} \cdot \mathrm{T}\right)+\left(2.68536 \times 10^{-5} \cdot \mathrm{T}^{2}\right)\right. \\
\left.-\left(2.42139 \times 10^{-8} \cdot \mathrm{T}^{3}\right)\right) \times 4.1868 \times 10^{3}
\end{gathered}
$$

Table A.2: Specific heat of water at varying temperatures.

\begin{tabular}{|c|c|}
\hline $\begin{array}{c}\text { Temperature. } \mathbf{T} \\
\left({ }^{\circ} \mathbf{C}\right)\end{array}$ & Specific Heat $\mathbf{c}_{\mathbf{p}, \mathbf{J} / \mathbf{k g} \cdot \mathbf{K}}$ \\
\hline 4.4 & 4199 \\
\hline 15.6 & 4185 \\
\hline 26.7 & 4177 \\
\hline 37.8 & 4174 \\
\hline 48.9 & 4176 \\
\hline 60.0 & 4181 \\
\hline 71.1 & 4191 \\
\hline 82.2 & 4199 \\
\hline 93.3 & 4211 \\
\hline
\end{tabular}




\section{A.3 Dynamic viscosity}

Table A.3 shows the dynamic viscosity of water from temperatures of 0 to $96.9^{\circ} \mathrm{C}$ gathered from Touloukian set of data [1970].

Table A.3: Dynamic viscosity of water at varying temperatures.

\begin{tabular}{|c|c|}
\hline $\begin{array}{c}\text { Temperature. } \mathrm{T} \\
\left({ }^{\circ} \mathrm{C}\right)\end{array}$ & $\begin{array}{c}\text { Dynamic Viscosity } \mu, \mathrm{kg} / \mathrm{m} \cdot \mathrm{s} \\
\left(\mathrm{x10}^{-3}\right)\end{array}$ \\
\hline 0 & 1.753 \\
\hline 6.85 & 1.422 \\
\hline 16.9 & 1.043 \\
\hline 26.9 & 0.823 \\
\hline 36.9 & 0.672 \\
\hline 46.9 & 0.56 \\
\hline 56.9 & 0.476 \\
\hline 66.9 & 0.411 \\
\hline 76.9 & 0.36 \\
\hline 86.9 & 0.319 \\
\hline 96.9 & 0.285 \\
\hline
\end{tabular}




\section{A.4 Thermal diffusivity}

The data that was collected from Touloukian set of data [1970] based which was reported to have an error of less than 0.1 percent and are displayed on Table A.4.

Table A.4: Thermal diffusivity of water at varying temperatures.

\begin{tabular}{|c|c|}
\hline Temperature. $\mathbf{T}\left({ }^{\circ} \mathrm{C}\right)$ & $\begin{array}{c}\text { Thermal diffusivity } \alpha, m^{2} / s \\
\left(\times 10^{-7}\right)\end{array}$ \\
\hline 4.85 & 1.350 \\
\hline 17.65 & 1.423 \\
\hline 18.35 & 1.43 \\
\hline 18.85 & 1.431 \\
\hline 19.25 & 1.431 \\
\hline 20.15 & 1.437 \\
\hline 21.45 & 1.445 \\
\hline 21.65 & 1.446 \\
\hline 22.85 & 1.447 \\
\hline 22.95 & 1.454 \\
\hline 23.35 & 1.454 \\
\hline 24.05 & 1.456 \\
\hline 25.35 & 1.465 \\
\hline
\end{tabular}




\section{Appendix B: Equipment}

\section{B.1 Specification of interferometer and equipment}

Table B.1 demonstrates the specification of the optical components used in the Mach-Zehnder interferometer.

Table B.1: Specification of the optical component in the interferometer.

\begin{tabular}{|c|c|c|c|c|}
\hline Component & Beam Splitter & Flat Mirror & $\begin{array}{l}\text { Parabolic } \\
\text { Mirror }\end{array}$ & $\begin{array}{l}\text { Spherical } \\
\text { Mirror }\end{array}$ \\
\hline $\begin{array}{l}\text { Size } \\
\text { (diameter) }\end{array}$ & $8 "$ & $8 ”$ & $8 "$ & $8 "$ \\
\hline Thickness & $1.5^{\prime \prime}$ & $1.50^{\prime \prime}$ & $1.37^{\prime \prime}$ & $1.37^{\prime \prime}$ \\
\hline Flatness & $1 / 20 \lambda$ & $1 / 20 \lambda$ & - & - \\
\hline Parallelism & 1 arc second & - & & \\
\hline Coated & $\begin{array}{l}\text { Side 1: } 50 \% \text { refractive } \\
\text { Side } 2: 50 \% \text { transitive }\end{array}$ & $95 \%$ reflective & & \\
\hline Aperture & - & - & $\mathrm{f} / 8.00$ & $\mathrm{f} / 3.00$ \\
\hline $\begin{array}{l}\text { Surface } \\
\text { Accuracy }\end{array}$ & & & $1 / 8 \lambda$ & $1 / 4 \lambda$ \\
\hline
\end{tabular}




\section{Appendix C: Mechanical drawing of the model}

In Chapter 3, the overall experimental model was explained in detail and shown in Figure 3.4 3.6. The details of each individual component were briefly discussed to understand the purpose. Figures C.1 - C.6 are the mechanical drawing of each individual parts with all the dimensions and tolerance required for the machining process. All of materials required for project were available at Master-Carr, and the three thermocouples that were used was purchased from Omega Engineering Canada. All the dimensions shown in the drawing are in $\mathrm{mm}$ with the scale provided in each figure.

Table C.1: Bill of material for the model.

$\left.\begin{array}{|l|c|}\hline \multicolumn{1}{|c|}{\text { Part Name }} & \text { Quantity } \\ \hline \text { Delrin Acetal Resin } & \\ \hline \text { 6061 Aluminum } & 4 \\ \hline \text { 4-40 Thread Size, 1in long Aluminum Socket } \\ \text { head screw }\end{array}\right)$


Table C.2: Fasteners used to assemble the model.

\begin{tabular}{|l|c|c|c|}
\hline Location & Tool and Size (in) & Conversion to mm & Tolerance $( \pm \mathbf{m m})$ \\
\hline$(1,2,3)$ Thermocouple hole & $1 / 32$ Drill bit & 0.80 & 0.5 \\
\hline (1) Socket (Thread) & $3 / 64$ Drill bit & 2.38 & 0.5 \\
\hline (1) Socket (Head) & $7 / 64$ Drill bit & 2.80 & 0.5 \\
\hline (2) Socket (Thread) & $1 / 8$ Drill bit & 3.18 & 0.5 \\
\hline (3) Socket (Thread) & $1 / 8$ Drill bit & 3.18 & 0.5 \\
\hline (3) Socket (Head) & Endmill & 5 & 0.5 \\
\hline
\end{tabular}

The optical frame position at the back end (shown in Figure C.6) is identical to the optical window positioned in the front that was describe in Chapter 2 with one minor change. The difference is the extrusion for the nuts for all four bolts. Since all three layers along the $\mathrm{z}$ directions required to be mated, a nut was required to secure the mate and prevent any damages to the model when assembled. 


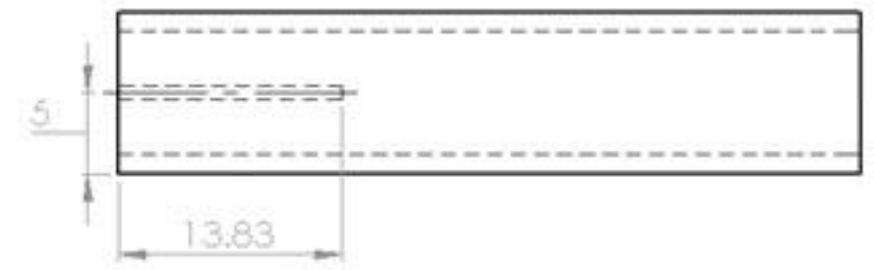

TOP VIEW
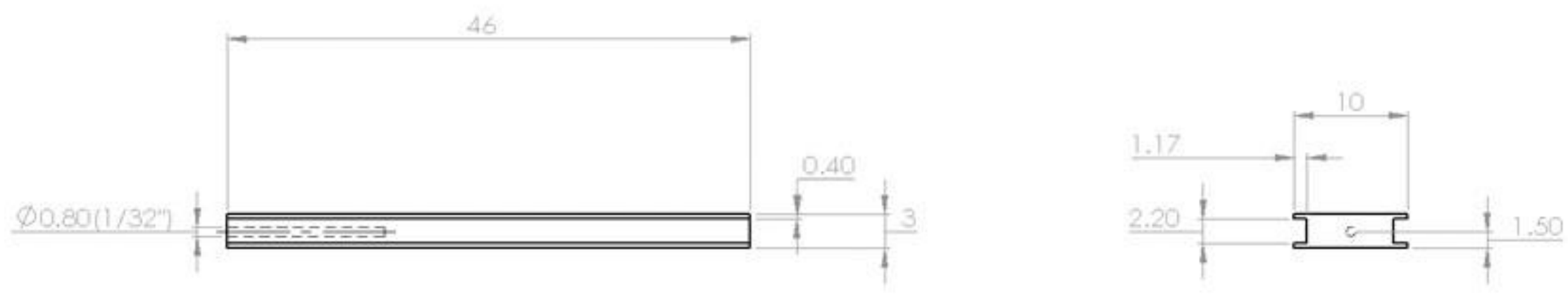

FRONT VIEW

SIDE VIEW

SCALE 2:1

Figure C.1: Aluminum barrier. 


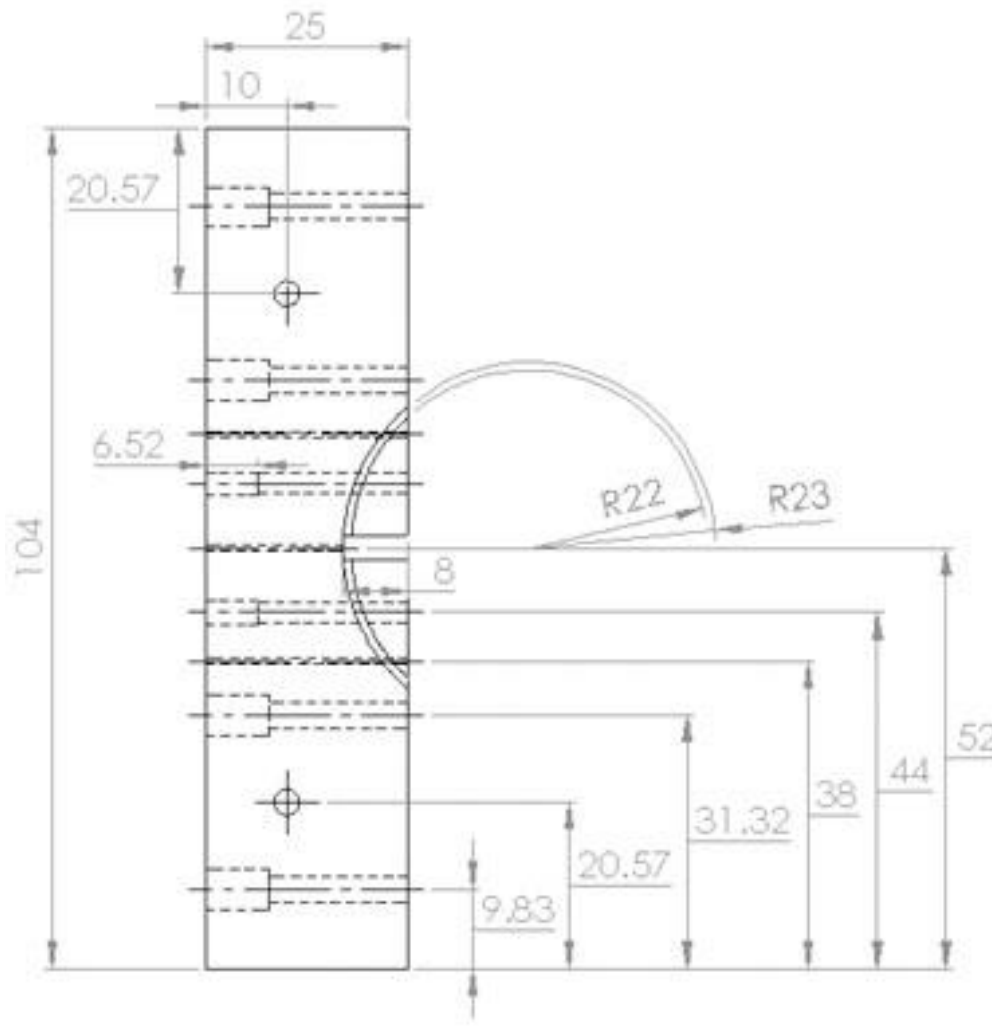

TOP VIEW

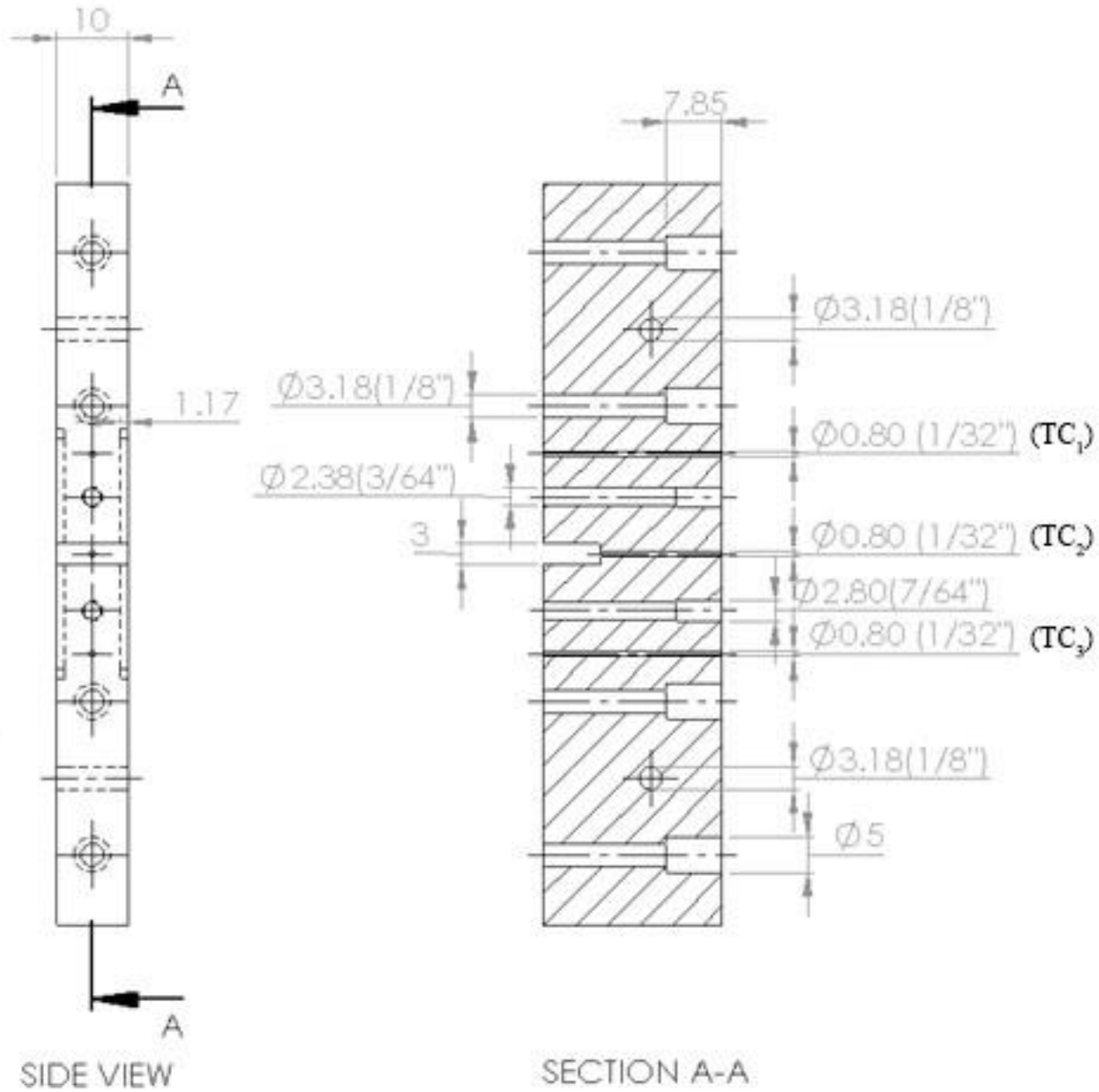

Figure C.2: Delrin side block (with thermocouples). 


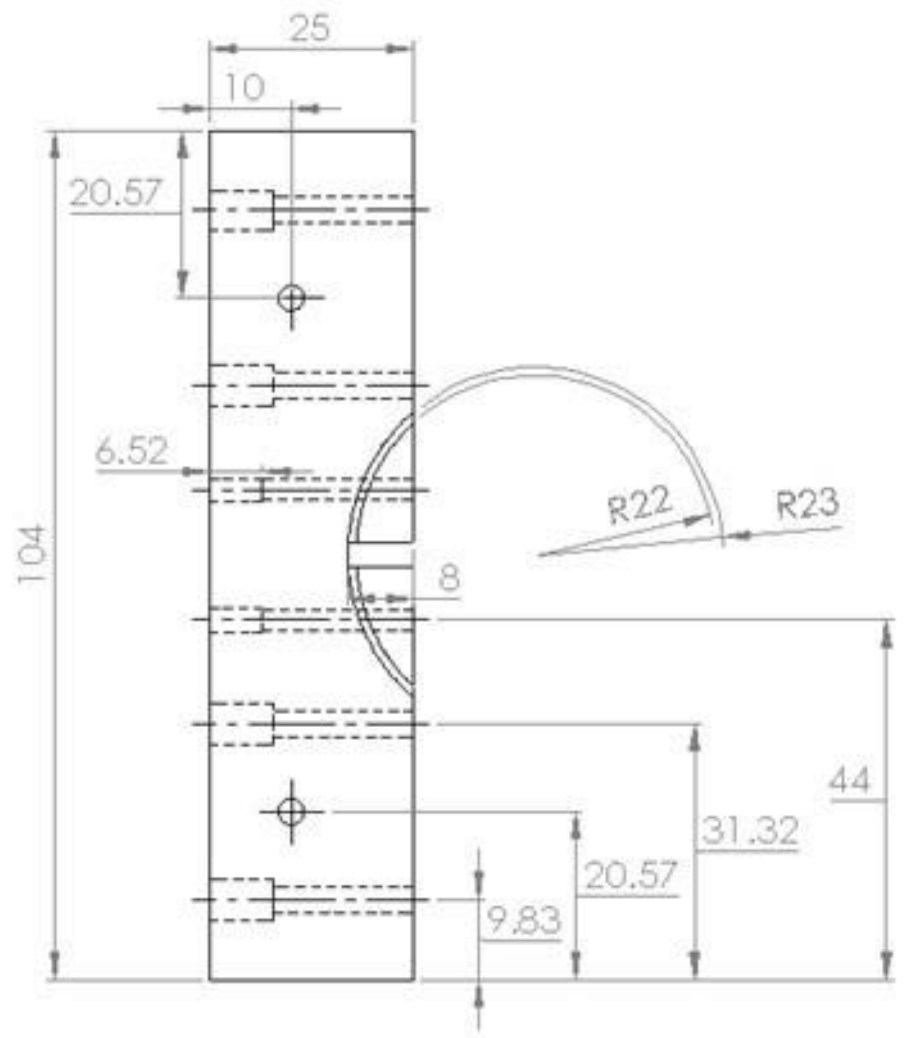

TOP VIEW

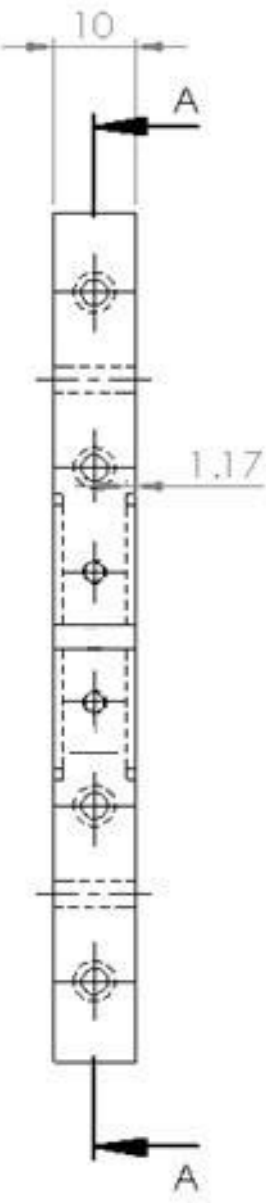

SIDE VIEW

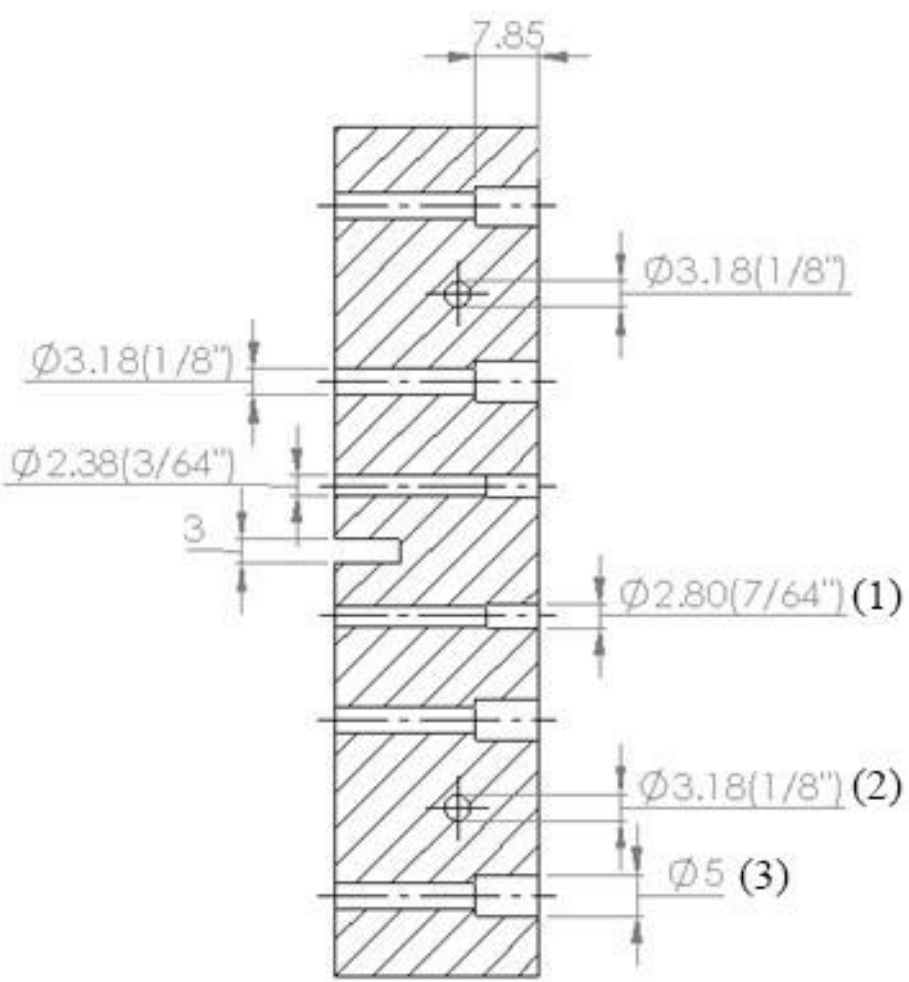

SECTION A-A

Figure C.3: Delrin side block (without thermocouple). 


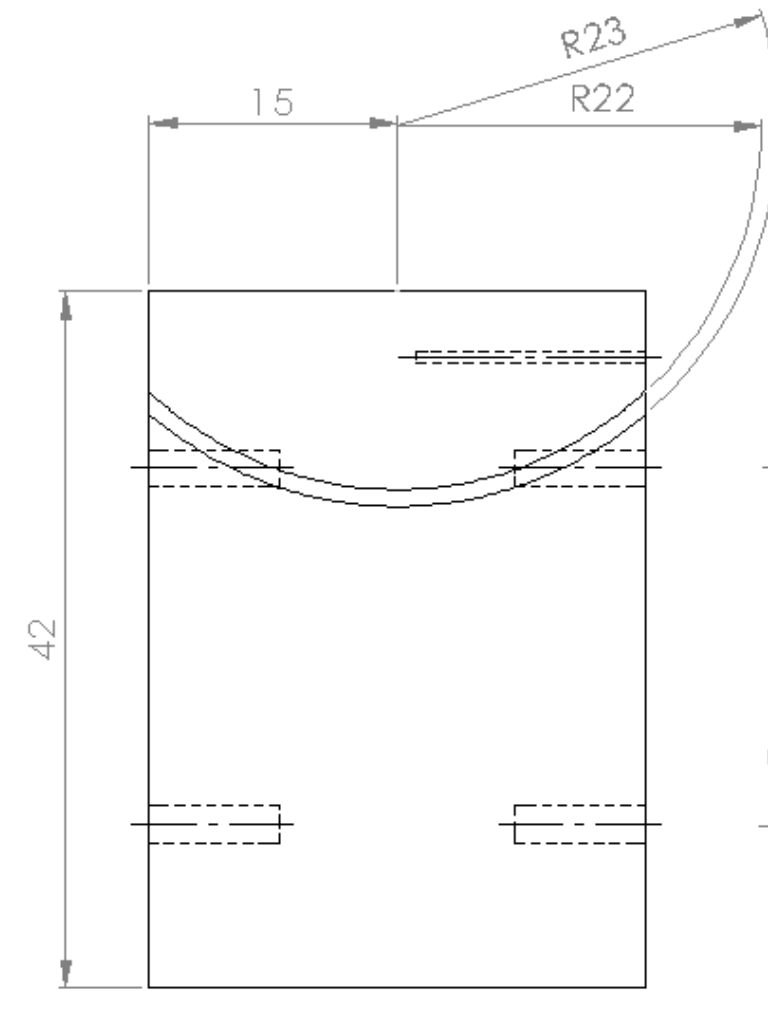

TOP VIEW

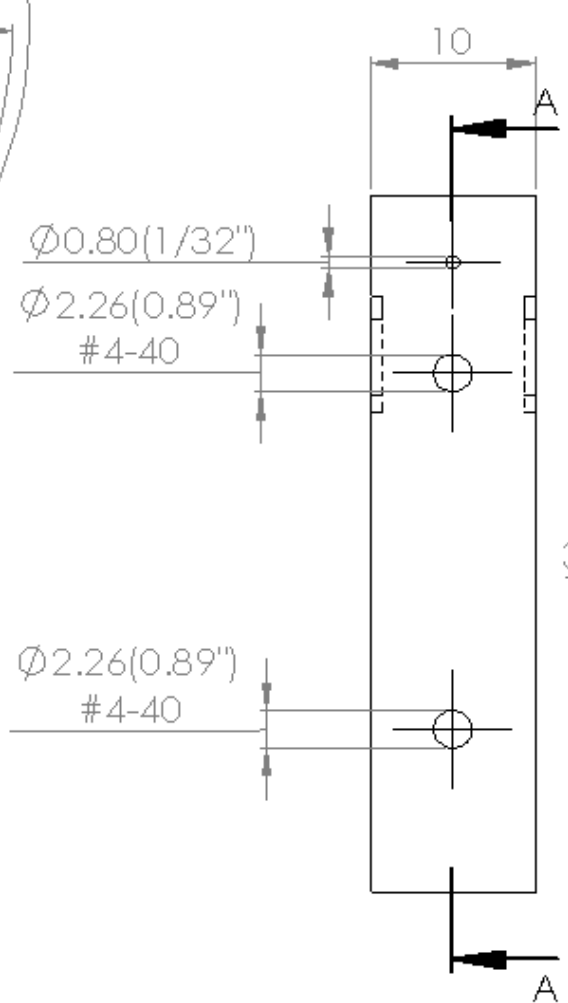

SIDE VIEW

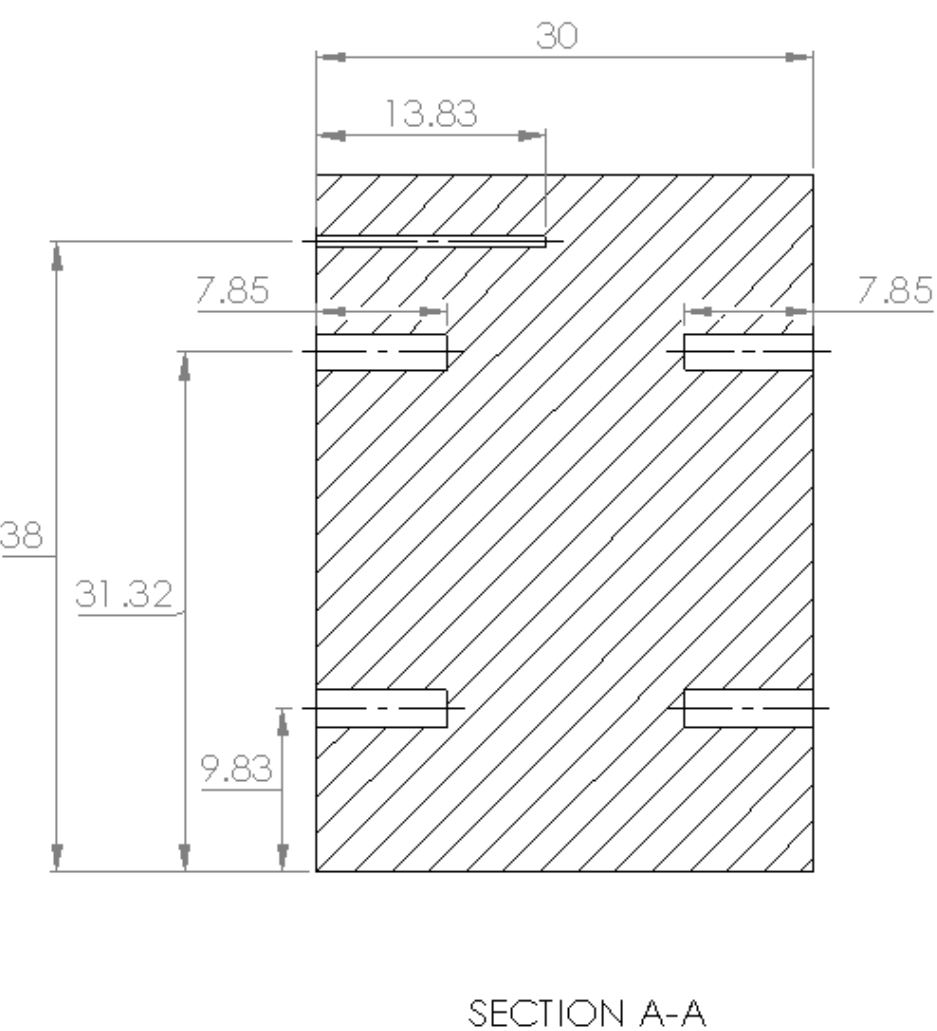

SECTION A-A

Figure C.4: Aluminum hot/cold block. 


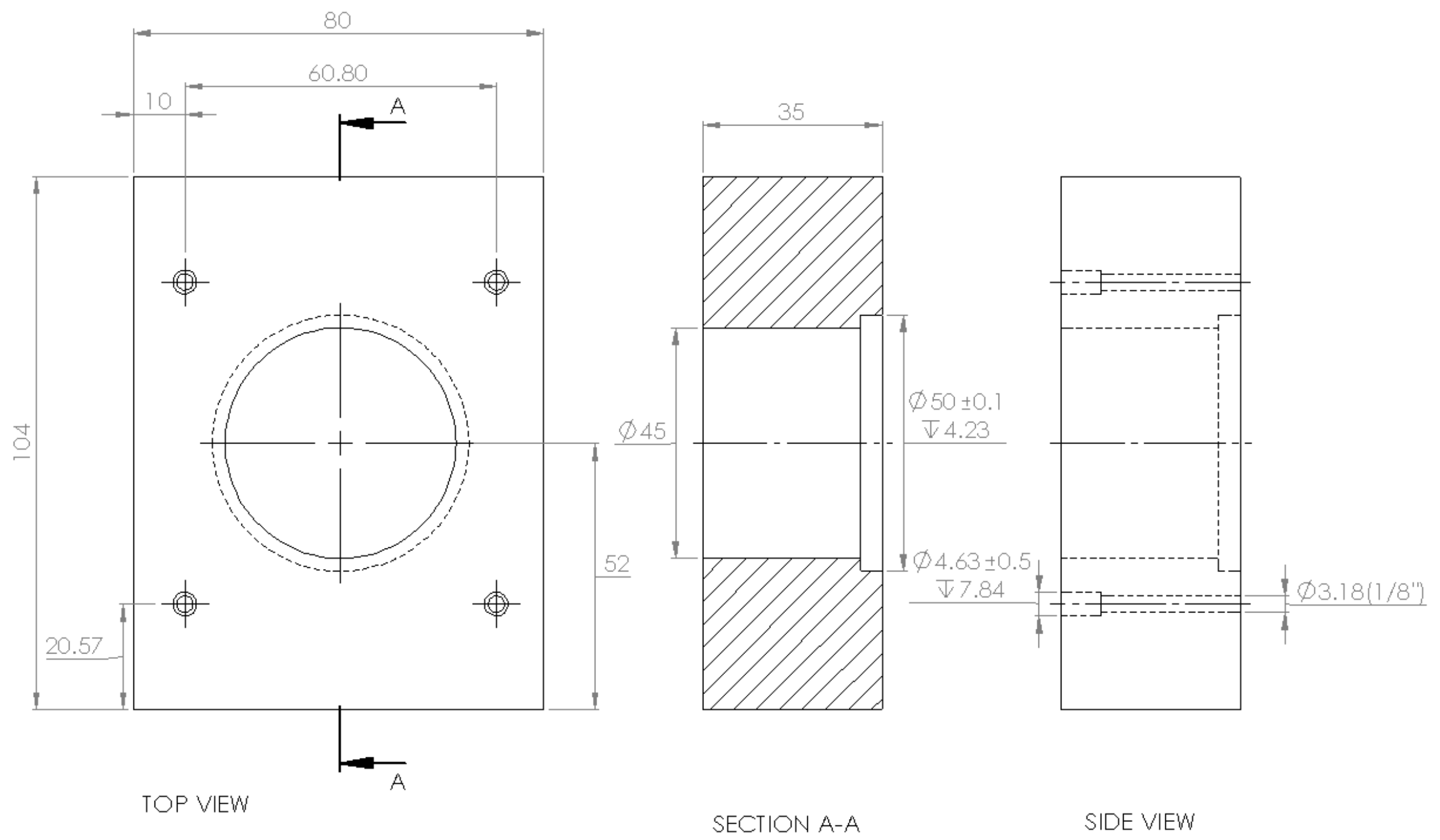

Figure C.5: Delrin optical frame (front). 


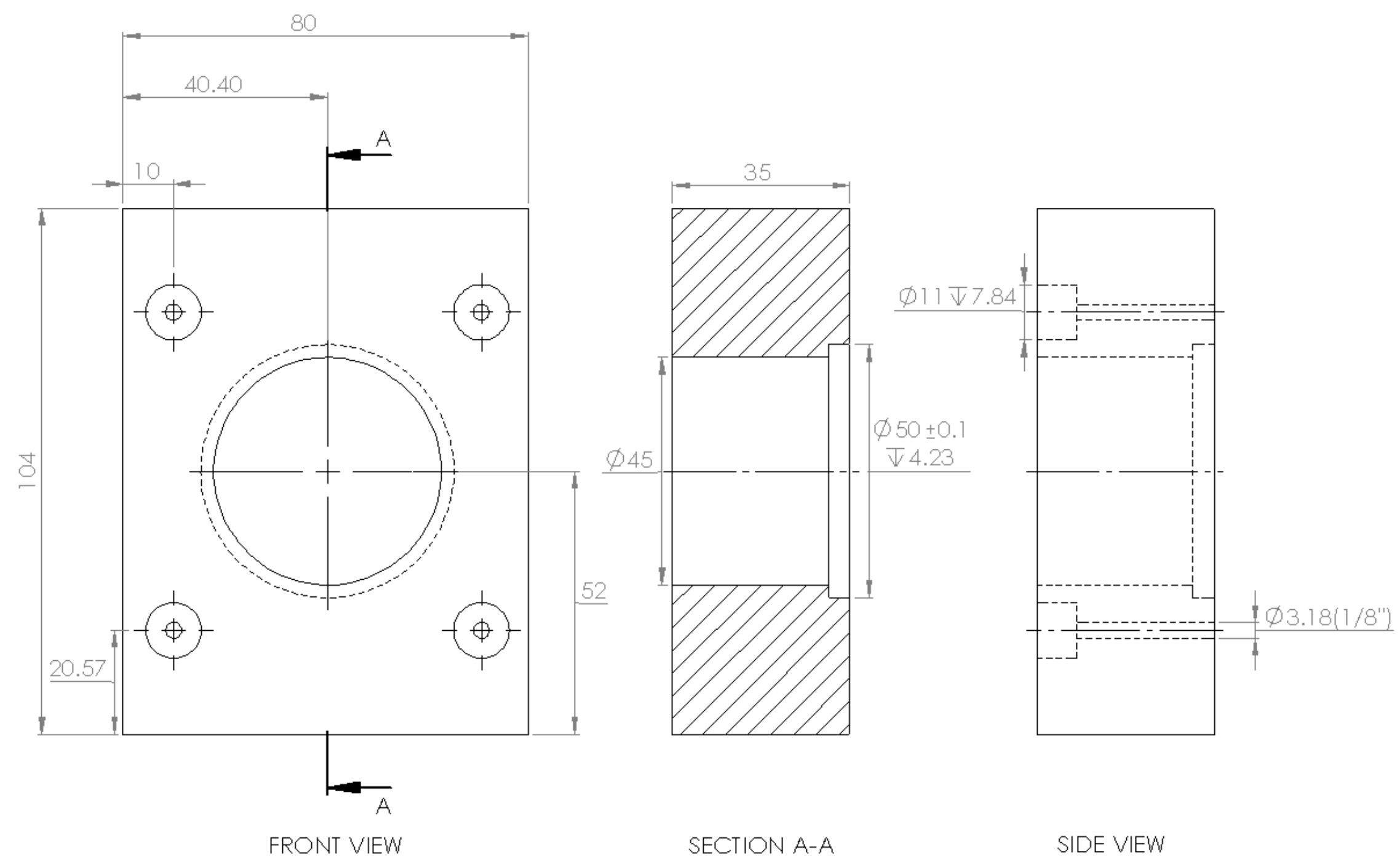

Figure C.6: Delrin optical frame (back). 


\section{Appendix D: Detailed experimental procedure}

\section{Still image camera procedure}

The camera setting can be reference to Derek Roeleveld thesis [2013]

1. The spherical mirror is aligned with the output from BS2. The light will focus to a point, which is the location the small flat mirror must be placed. The light should be reflected by $90^{\circ}$ towards the camera position.

2. The camera should be moved as close to reflected light from the small flat mirror to enlarge the image that will be captured. Move the camera further away from the output of the reflected light until both fluid domains of the model can be seen in the camera's view.

3. The laser is turned off, and a light source is placed at the location the model is placed. The camera was then focused on the light source to reduce refraction error, by adjusting the bellows on the camera stand.

4. After the camera was focused, the light source was removed, and the laser was turned on.

5. Turn the computer on, and ensure the camera is connected to the computer. Open the Capture One Pro program, and set the setting corresponding to Roeleveld thesis [2013] in still image camera procedure.

6. A test image should be taken at this point. If indication of overexposure was highlighted by the software, the neutral density filter should be placed in front of the laser. As well, if there is any imperfection that can be seen in the captured test image, the small mirror should be relocated. There is small imperfection on the surface of the small flat mirror, which are areas the focused light should avoid.

7. Set both water baths to ambient temperature and wait for 10 minutes till the digital multimeters displays each thermocouple to be at ambient temperature. The first image to be taken was the unheated image and should be labeled as "Unheated model at $20^{\circ} \mathrm{C}$ " plus the type of fringe setting used in the software.

8. The shutter is closed, the camera is cocked, the lights in the room should be turned off, and photograph was taken of the unheated model.

9. The lights are turned on, and the water baths should be changed to reach the desired temperature difference for the heated model. After 20 minutes, check the digital multimeter 
and make sure the barrier is at $20^{\circ} \mathrm{C}$, and the temperature of the hot and cold block are at the specific temperature to reach the desired temperature difference. If not, slightly adjust the water bath's temperature and wait till the temperature of all three component are met.

10. Change the title of the next image to be captured as "Heated model with temperature difference of __ ${ }^{\circ} \mathrm{C}$ " plus the type of fringe setting used. Repeat step 8 to capture the image of the heated model.

11. The lights are turned on, and carefully remove the model from the support frame without disturbing the surrounding setup. A scale photo must be taken. A vertical scale reference was placed in position where the model was placed. Step 8 was repeated to capture the scale photo.

12. The water baths, and the digital multimeter can be turned off. The picture needs to be processed and exported in Capture One Pro. With the setting being the same in Roeleveld thesis [2013], under the "Quick" tab, press the "process" button to export the captured image as an 8-bit TIFF image.

13. The exported image that should be in the designated folder will need to be edited on Photoshop. Using Photoshop, convert image to a black and white image. Save the image file.

14. The final step was to transfer TIFF image to a custom MATLAB image processing to extract the average fringe gradient of both base and test fluids. 


\section{Appendix E: Sample calculation}

A set of experimental calculations will be presented to demonstrate how the heat transfer results were obtained.

\section{E.1 Given data}

\begin{tabular}{|c|c|}
\hline Variable & Value \\
\hline Hot plate surface temperature $\left(\mathrm{T}_{\mathrm{H}}\right)$ & $25^{\circ} \mathrm{C}$ \\
\hline Cold plate surface temperature $\left(T_{C}\right)$ & $15^{\circ} \mathrm{C}$ \\
\hline Temperature Difference, $\Delta \mathrm{T}$ & $10 \mathrm{~K}$ \\
\hline Ambient Temperature $\left(T_{\infty}\right)$ & $20^{\circ} \mathrm{C}$ \\
\hline Gravitational acceleration $(\mathrm{g})$ & $9.81 \mathrm{~m} / \mathrm{s}^{2}$ \\
\hline Thickness of the fluid domain (tw) & $0.0085 \mathrm{~m}$ \\
\hline Thickness of the barrier (tb) & $0.003 \mathrm{~m}$ \\
\hline Thermal conductivity of water at $20^{\circ} \mathrm{C}$ & $0.597 \mathrm{~W} / \mathrm{mK}$ \\
\hline Path length (L) & $0.01 \mathrm{~m}$ \\
\hline Width of the barrier (w) & $0.03 \mathrm{~m}$ \\
\hline Wavelength of He-Ne laser $(\lambda)$ & $6.328 \times 10^{-7} \mathrm{~m}$ \\
\hline Density of $\mathrm{SiO}_{2}$ nanoparticle $\left(\rho_{\mathrm{SiO}_{2}}\right)$ & $2650 \mathrm{~kg} / \mathrm{m}^{3}$ \\
\hline $\begin{array}{l}\text { Temperature coefficient of refractive index of water at } 20^{\circ} \mathrm{C} \\
\text { [Naylor, 2017] }\left(\frac{d n}{d T_{H_{2}} \mathrm{O}}\right)\end{array}$ & $9.6 \times 10^{-5} K^{-1}$ \\
\hline $\begin{array}{l}\text { Temperature coefficient of refractive index of } \mathrm{SiO}_{2} \text { at } 20^{\circ} \mathrm{C} \text { with } \\
10 \% \text { by volume concentration [Yousefi, 2019] }\left(\frac{d n}{d T}_{H_{2} O}\right)\end{array}$ & $5.15 \times 10^{-5} K^{-1}$ \\
\hline
\end{tabular}




\section{E.2 Convert weight concentration to volume concentration}

The commercially prepared nanofluid was available in a specified weight concentration. The results presented in this thesis were obtained at specified volume concentrations. A conversion of the commercially prepared nanofluid given as $40 \%$ by weight concentration will be converted into volume concentration using equation E.1.

$$
\begin{gathered}
\phi=\frac{\omega \cdot \rho_{w}}{\left(1-\frac{\omega}{100}\right) \cdot \rho_{S_{i O}}+\left(\frac{\omega}{100}\right) \cdot \rho_{w}} \\
\phi=\frac{40 \cdot\left(1000 \frac{\mathrm{kg}}{\mathrm{m}^{3}}\right)}{\left(1-\frac{40}{100}\right) \cdot\left(2650 \frac{\mathrm{kg}}{\mathrm{m}^{3}}\right)+\left(\frac{40}{100}\right) \cdot\left(1000 \frac{\mathrm{kg}}{\mathrm{m}^{3}}\right)}=20.1 \%
\end{gathered}
$$

\section{E.3 Nanofluid sample preparation}

Each sample nanofluid solution prepared needed to be $50 \mathrm{ml}$ in volume. Since the concentration of the commercial prepared nanofluid was known, the process to achieve the desired volume concentration of a total of $50 \mathrm{ml}$ was done using equation (E.2). A sample calculation demonstrates the amount of deionized water needed to dilute the commercial prepared nanofluid to $50 \mathrm{ml}$ of $15 \%$ by volume concentration solution.

$$
\begin{gathered}
C_{i} V_{i}=C_{F} V_{F} \\
V_{i}=\frac{0.15 * 50 \mathrm{ml}}{0.20}=37.5 \mathrm{ml}
\end{gathered}
$$


The difference between the amounts of commercially prepared nanofluid to make $50 \mathrm{ml}$ of the $15 \%$ by volume concentration was the amount of deionized water needed to be added. Thus, 12.5 $\mathrm{ml}$ of deionized water was added to $37.5 \mathrm{ml}$ of $\mathrm{SiO}_{2}$-water nanofluid (20\% by volume concentration).

\section{E.4 Predict the total fringe shift}

As discussed in Chapter 4, the total fringe shift was required to be calculated to understand the total number of fringes that will be seen in the model using the interferometer. The thermal coefficient of refractive index of water is approximately $10^{-4} \mathrm{~K}^{-1}$.

The total fringe shift equations are as follows:

$$
\Delta \varepsilon=\frac{\mathrm{L}}{\lambda} \frac{\partial \mathrm{n}}{\partial \mathrm{T}} \Delta \mathrm{T}=\frac{(0.01 \mathrm{~m})}{632.8 \times 10^{-9} \mathrm{~m}} \times\left(10^{-4} \frac{1}{\mathrm{~K}}\right) \times 10 \mathrm{~K}=16 \text { fringes }
$$

The total fringe shift available within a path length of $0.01 \mathrm{~m}$ will be 16 fringes within the fluid domain. Comparing the prediction to the fringes seen in the experiment, the total number of fringes holds true when compared to figure $5.11 \mathrm{~b}$ of the heated model.

\section{E.5 Surface temperature (temperature of the barrier)}

The surface temperature that will be measured from the experimental

$$
T_{b}=\frac{T_{H}+T_{C}}{2}=\frac{25^{\circ} \mathrm{C}+15^{\circ} \mathrm{C}}{2}=20^{\circ} \mathrm{C}
$$

\section{E.5 Scale factor}

The image that will be recorded will require a scale factor to accommodate the pixel dimensions. Since all of the measurement will be along the $\mathrm{x}$-axis. The scale factor calculated will be along the vertical direction $\left(\mathrm{SF}_{\mathrm{v}}\right)$. The number of pixels within two pins captured within the model's position was 863.15 pixels (represented by $\mathrm{N}_{\text {pixel }}$ ). The distance between the tips of the pins were measured to be 0.2738 in, which is equal to $0.006954 \mathrm{~m}$ (represented as $\mathrm{D}_{\text {actual }}$ ). Using photoshop, the number of pixels within the pin from the capture image can be measured $\left(\mathrm{N}_{\text {pixel }}\right)$ 
The scale factor can be calculated along the vertical direction.

$$
S F_{v}=\frac{D_{\text {actual }}}{N_{\text {pixel }}}=\frac{0.006954 \mathrm{~m}}{863.15}=8.05 \times 10^{-6} \frac{\mathrm{m}}{\text { pixels }}
$$

\section{E.6 Extracting fringe gradient from scanning algorithm}

The information outputted by the scanning algorithm was the number of pixels per fringe measured between the first peak to peak of the sine wave. This data will be used to compute the fringe gradient, which is done in two steps. Table G.2 and G.6 shows the data gathered from the scanning algorithm. Firstly, the number of pixels per fringe from the measured peak to peak will need to be inversed (fringe/pixel). The inverse of the peak to peak is the fringes per pixel. The number of pixels per fringe in a measured peak to peak from the heated model experiment with water in both fluid domains was 154 pixel/fringe.

$$
\frac{d \varepsilon}{d p}=\frac{1}{d p / d \varepsilon}=\frac{1}{154.36 \frac{\text { pixel }}{\text { fringe }}}=6.48 \times 10^{-3} \frac{\text { fringe }}{\text { pixel }}
$$

To convert the data to fringes per meter, the inverse peak to peak is divided by the scale factor $\left(\mathrm{SF}_{\mathrm{v}}\right)$, resulting in the fringe gradient (fringe/m). Table G.3 and G.6 demonstrates the inverse of the data outputted by the scanning algorithm.

$$
\frac{d \varepsilon}{d x}=\frac{\frac{d \varepsilon}{d p}}{S F_{v}}=\frac{6.48 \times 10^{-3} \frac{\text { fringe }}{\text { pixel }}}{8.05 \times 10^{-6} \frac{\mathrm{m}}{\text { pixels }}}=804 \frac{\text { fringe }}{\mathrm{m}}
$$

For experiments performed in infinite fringe mode, the heated model was the data only used to compute the relative thermal conductivity aside from the approximated fringe gradient correction due to deflection. A table of the raw, inverse of the raw data, and the fringe gradient are shown in Tables G.6 and G.7. 


\section{E.7 Subtraction technique}

Since both fringe settings were experimented with deionized water in both fluid domains, a sample calculation of both fringe settings will be shown. The gathered data are performed the same way, as well as the processing method to extract the average fringe gradient. However, as discussed in

Chapter 5, the subtraction technique was different for both fringe settings.

\section{Finite fringe setting}

Using equation (5.14) (subtraction technique for finite fringe mode), the fringe gradient at the barrier due to the temperature field can be computed. The sample calculation shown below was the base fluid's fringe gradient. Table G.5 show the measured fringe gradient for experiments performed with water in both fluid domains in finite fringe mode.

$$
\frac{d \varepsilon}{d x_{b_{T}}}=1810 \frac{\text { fringe }}{\mathrm{m}}-804 \frac{\text { fringe }}{\mathrm{m}}=1006 \frac{\text { fringe }}{\mathrm{m}}
$$

\section{Infinite fringe setting}

The measured data performed with water in both fluid domains set in infinite fringe mode are shown in Table G.6. By referencing Figure 5.8, the approximated fringe gradient correction due to deflection are shown for both fluid domains. Using equation (5.15) (subtraction technique for infinite fringe mode), the sample calculation of the base fluid's fringe gradient measured at the barrier due to the temperature field is as follows: Table G. 8 show the measured fringe gradient for experiments performed with water in both fluid domain in infinite fringe mode. For example, the corrected fringe gradient for the base (top) fluid domain is:

$$
\frac{d \varepsilon}{d x_{T}}=710 \frac{\text { fringe }}{\mathrm{m}}+6 \frac{\text { fringe }}{\mathrm{m}}=716 \frac{\text { fringe }}{\mathrm{m}}
$$

\section{E.8 Relative thermal conductivity of the test fluid}

The process of computing the relative thermal conductivity of the test fluid was done by using equation (5.16) as discussed in Chapter 5. The data used in the sample calculation is from an 
experimented testing $\mathrm{SiO}_{2}$ - water nanofluid at $10 \%$ by volume concentration (Table G.10). The information needed to compute the relative thermal conductivity of the nanofluid in the test fluid domain is the temperature coefficients of refractive index of both test and base fluids $\left(\frac{d n}{d T}_{\mathrm{SiO}_{2}} \&{\frac{d n}{d T_{H_{2}} \mathrm{O}}}\right)$, the measured fringe gradients from both fluid domains at the barrier surfaces $\left(\frac{d \varepsilon}{d x_{\mathrm{SiO}_{2}}} \& \frac{d \varepsilon}{d x} \mathrm{H}_{2} \mathrm{O}\right)$. The average values of $\frac{d \varepsilon}{d x_{\mathrm{SiO}_{2}}}$ and $\frac{d \varepsilon}{d x_{\mathrm{H}_{2} \mathrm{O}}}$ in Table G.10 are used in the calculation, as follows:

$$
\frac{k_{\mathrm{SiO}_{2}}}{k_{\mathrm{H}_{2} \mathrm{O}}}=\frac{\left(\left.\frac{d \varepsilon}{d x}\right|_{\mathrm{SiO}_{2}}\right)\left(\frac{d n}{d T_{\mathrm{H}_{2} \mathrm{O}}}\right)}{\left(\left.\frac{d \varepsilon}{d x}\right|_{\mathrm{H}_{2} \mathrm{O}}\right)\left(\frac{d n}{d T_{\mathrm{SiO}_{2}}}\right)}=\frac{\left(704.1 \frac{\text { fringe }}{m}\right)\left(9.6 \times 10^{-5}\right)}{(706.9)\left(5.15 \times 10^{-5}\right)}=1.86
$$

The equation is simplified further for experiments for with deionized water in both fluid domains as discussed in Chapter 5. The temperature coefficient of refractive index can be omitted from the equation because the fluid in both domains are the same. Thus, the relative thermal conductivity of the deionized water is as followed (Table G.5):

$$
\frac{k_{\mathrm{H}_{2} \mathrm{O}}}{k_{\mathrm{H}_{2} \mathrm{O}}}=\frac{1039 \frac{\text { fringe }}{m}}{1013 \frac{\text { fringe }}{m}}=1.026
$$




\section{Appendix F: Uncertainty Analysis}

Table F.1: Summary of sources of uncertainty.

\begin{tabular}{|c|c|c|}
\hline Variable & $\begin{array}{c}\text { Absolute } \\
\text { Uncertainty }\end{array}$ & $\begin{array}{c}\text { Percent } \\
\text { Uncertainty }\end{array}$ \\
\hline Hot/Cold temperature & $\pm 0.1 \mathrm{~K}$ & $\pm 0.5 \%$ \\
\hline Barrier temperature, $\mathrm{T}_{\mathrm{b}}$ & $\pm 0.1 \mathrm{~K}$ & $\pm 0.5 \%$ \\
\hline Specific heat of water, $\left(\mathrm{c}_{\mathrm{p}}\right)$ & & $\pm 0.25 \%$ \\
\hline Dynamics viscosity of water, $(\mu)$ & & $\pm 0.5 \%$ \\
\hline Thermal conductivity of water, $(\mathrm{k})$ & $\pm 0.141 \mathrm{~K}$ & $\pm 2.83 \%$ \\
\hline Temperature difference $(\Delta T)$ & $\pm 1 \times 10^{-4} \mathrm{~m}$ & $\pm 1.18 \%$ \\
\hline Length of the fluid domain, $(\mathrm{H})$ & & $\pm 4.5 \%$ \\
\hline Measured fringe gradient with water in finite fringe & & \\
\hline setting for both bf and tf, $\left(\frac{\partial \epsilon}{\partial x}\right)$ & & \\
\hline Measured fringe gradient with water in infinite fringe & & \\
\hline setting for bf and tf, $\left(\frac{\partial \epsilon}{\partial x}\right)$ & & \\
\hline
\end{tabular}

\section{F.1 Uncertainty in the absolute thermal conductivity}

The technique used to evaluate the uncertainty is from Kline and McClintock [1953]. The uncertainty of the measured variable is based on the power of each individual experimental measured variable in the equation. The variable $w$ in the uncertainty analysis is denoted as uncertainty in the independent variable. Equation (F.1) is in the form of a product function and can be simplified as follows: 


$$
k_{t f}=\left(k_{w}\right)^{1}\left(\left.\frac{\partial \epsilon}{\partial x}\right|_{b f}\right)^{-1}\left(\left.\frac{\partial n}{\partial T}\right|_{t f}\right)^{-1}\left(\left.\frac{\partial \epsilon}{\partial x}\right|_{t f}\right)^{1}\left(\left.\frac{\partial n}{\partial T}\right|_{b f}\right)^{1}
$$

The resulting uncertainty equation is:

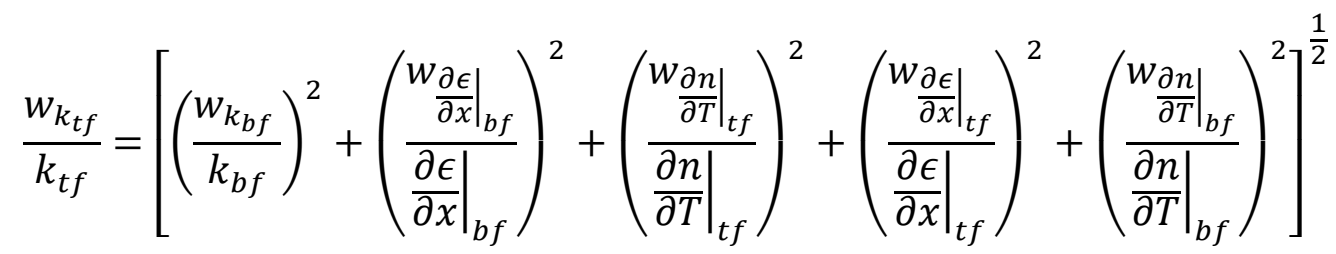

Uncertainty performed in finite fringe mode with water in both fluid domains:

Performing with water in both fluid domains allows for equation F.2 to be reduced by neglecting the temperature coefficient of refractive index of water, and the uncertainty is as followed:

$$
\frac{w_{k_{t f}}}{k_{t f}}=\left[\left(\frac{w_{k_{b f}}}{k_{b f}}\right)^{2}+\left(\frac{\left.w_{\frac{\partial \epsilon}{\partial x}}\right|_{b f}}{\left.\frac{\partial \epsilon}{\partial x}\right|_{b f}}\right)^{2}+\left(\frac{\left.w_{\frac{\partial \epsilon}{\partial x}}\right|_{t f}}{\left.\frac{\partial \epsilon}{\partial x}\right|_{t f}}\right)^{2}\right]^{1 / 2}=\left[1.0^{2}+4.44^{2}+4.44^{2}\right]^{\frac{1}{2}}= \pm 6.35 \%
$$

Uncertainty performed in infinite fringe mode with water in both fluid domains:

$$
\frac{w_{k_{t f}}}{k_{t f}}=\left[\left(\frac{w_{k_{b f}}}{k_{b f}}\right)^{2}+\left(\frac{\left.w_{\frac{\partial \epsilon}{\partial x}}\right|_{b f}}{\left.\frac{\partial \epsilon}{\partial x}\right|_{b f}}\right)^{2}+\left(\frac{\left.w_{\frac{\partial \epsilon}{\partial x}}\right|_{t f}}{\left.\frac{\partial \epsilon}{\partial x}\right|_{t f}}\right)^{2}\right]^{1 / 2}=\left[1.0^{2}+5.32^{2}+5.32^{2}\right]^{\frac{1}{2}}= \pm 7.58 \%
$$




\section{Appendix G: Experimental data}

\section{G.1 Thermocouple calibrations}

Table B. 2 shows the thermocouple calibration performed with a calibrated glass thermometer using one of the temperature-controlled water baths.

Table G.1: Hot plate thermocouple calibration.

\begin{tabular}{|c|c|c|c|}
\hline \multirow[t]{2}{*}{ Actual Temperature } & \multicolumn{3}{|c|}{ Thermocouple Reading $\left({ }^{\circ} \mathrm{C}\right)$} \\
\hline & Hot $\left(\mathbf{T}_{\mathbf{H}}\right)$ & $\operatorname{Barrier}\left(\mathbf{T}_{\mathbf{F}}\right)$ & Cold $\left(\mathbf{T}_{\mathbf{C}}\right)$ \\
\hline 22.7 & 22.6 & 22.5 & 22.6 \\
\hline 22.2 & 22.3 & 22.2 & 22.1 \\
\hline 21.7 & 21.8 & 21.8 & 21.9 \\
\hline 21.2 & 21.1 & 21.0 & 21.1 \\
\hline 20.8 & 21.0 & 20.9 & 21.0 \\
\hline 20.2 & 20.3 & 20.3 & 20.2 \\
\hline 19.7 & 19.8 & 19.8 & 19.6 \\
\hline 19.2 & 19.2 & 19.1 & 19.1 \\
\hline 18.7 & 18.8 & 18.8 & 18.9 \\
\hline 18.3 & 18.4 & 18.3 & 18.2 \\
\hline 17.7 & 17.6 & 17.6 & 17.7 \\
\hline 17.7 & 17.5 & 17.5 & 17.3 \\
\hline 16.7 & 16.7 & 16.8 & 16.7 \\
\hline 16.3 & 16.3 & 16.3 & 16.3 \\
\hline Correction & 0.1 & 0.1 & 0.1 \\
\hline
\end{tabular}




\section{G.2 Water experiment}

\section{G.2.1 Finite fringe setting}

The raw data gathered from the scanning algorithm is shown in table G.2. The raw data is the scanned number of pixels per fringe measured from the first two fringes near the surface of the barrier for both fluid domains.

Table G.2: Data gathered from the scanning algorithm performed in finite mode with water in both fluid domains.

\begin{tabular}{|c|c|c|c|c|}
\hline \multirow{2}{*}{ Scanned location } & \multicolumn{2}{|c|}{ Base fluid domain (pixel/fringe) } & \multicolumn{2}{c|}{ Test fluid domain (pixel/fringe) } \\
\cline { 2 - 5 } & Unheated & Heated & Unheated & Heated \\
\hline 1 & 154 & 69 & 139 & 63 \\
\hline 2 & 160 & 69 & 138 & 64 \\
\hline 3 & 153 & 68 & 146 & 65 \\
\hline 4 & 157 & 69 & 147 & 65 \\
\hline 5 & 160 & 69 & 141 & 65 \\
\hline 6 & 159 & 68 & 140 & 65 \\
\hline 7 & 158 & 68 & 143 & 66 \\
\hline 8 & 157 & 68 & 143 & 65 \\
\hline 9 & 150 & 69 & 142 & 64 \\
\hline 10 & 152 & 69 & 139 & \\
\hline
\end{tabular}


Table G.3: Inversed measured data gathered from the scanning algorithm performed in finite fringe mode with water in both fluid domains.

\begin{tabular}{|c|c|c|c|c|}
\hline \multirow[t]{2}{*}{ Scanned location } & \multicolumn{2}{|c|}{$\begin{array}{l}\text { Base fluid domain (fringe/pixel) } \\
\qquad \times 10^{-3}\end{array}$} & \multicolumn{2}{|c|}{$\begin{array}{l}\text { Test fluid domain (fringe/pixel) } \\
\qquad \times 10^{-4}\end{array}$} \\
\hline & Unheated & Heated & Unheated & Heated \\
\hline 1 & 6.48 & 1.46 & 7.18 & 1.59 \\
\hline 2 & 6.25 & 1.44 & 7.24 & 1.55 \\
\hline 3 & 6.52 & 1.47 & 6.84 & 1.53 \\
\hline 4 & 6.36 & 1.45 & 6.79 & 1.53 \\
\hline 5 & 6.23 & 1.44 & 7.10 & 1.54 \\
\hline 6 & 6.29 & 1.46 & 7.14 & 1.53 \\
\hline 7 & 6.32 & 1.47 & 6.97 & 1.53 \\
\hline 8 & 6.39 & 1.47 & 7.01 & 1.51 \\
\hline 9 & 6.69 & 1.45 & 7.05 & 1.55 \\
\hline 10 & 6.59 & 1.46 & 7.17 & 1.56 \\
\hline
\end{tabular}


Table G.4: Measured fringe gradient data with water to water experiment performed in finite fringe mode.

\begin{tabular}{|c|c|c|c|c|}
\hline \multirow{2}{*}{ Scanned location } & \multicolumn{2}{|c|}{ Base fluid domain (fringe/m) } & \multicolumn{2}{c|}{ Test fluid domain (fringe/m) } \\
\cline { 2 - 5 } & Unheated & Heated & Unheated & Heated \\
\hline 1 & 804 & 1810 & 891 & 1971 \\
\hline 2 & 776 & 1790 & 899 & 1928 \\
\hline 3 & 810 & 1823 & 849 & 1901 \\
\hline 4 & 790 & 1798 & 843 & 1902 \\
\hline 5 & 773 & 1790 & 881 & 1906 \\
\hline 6 & 780 & 1818 & 886 & 1905 \\
\hline 7 & 784 & 1827 & 865 & 1896 \\
\hline 8 & 793 & 1823 & 871 & 1873 \\
\hline 9 & 830 & 1795 & 875 & 1940 \\
\hline 10 & 819 & 1811 & 890 & \\
\hline
\end{tabular}


Table G.5: Fringe gradient of the measured data for both water fluid domains using subtraction technique.

\begin{tabular}{|c|c|c|c|c|}
\hline Scanned location & $\begin{array}{l}\text { Base fluid } \\
\text { domain } \\
\text { (fringe/m) }\end{array}$ & $\begin{array}{c}\text { Base fluid } \\
\text { domain -Average } \\
\text { fringe gradient } \\
\text { (fringe } / \mathrm{m})\end{array}$ & $\begin{array}{c}\text { Test fluid domain } \\
\text { (fringe/m) }\end{array}$ & $\begin{array}{l}\text { Test fluid } \\
\text { domain - } \\
\text { Average } \\
\text { fringe } \\
\text { gradient } \\
\text { (fringe/m) }\end{array}$ \\
\hline 1 & 1006 & \multirow{10}{*}{1013} & 1080 & \multirow{10}{*}{1039} \\
\hline 2 & 1014 & & 1028 & \\
\hline 3 & 1014 & & 1052 & \\
\hline 4 & 1008 & & 1059 & \\
\hline 5 & 1016 & & 1025 & \\
\hline 6 & 1038 & & 1019 & \\
\hline 7 & 1043 & & 1031 & \\
\hline 8 & 1030 & & 1002 & \\
\hline 9 & 964 & & 1046 & \\
\hline 10 & 993 & & 1049 & \\
\hline
\end{tabular}




\section{G.2.2 Infinite fringe mode:}

Table G.6: Data gathered from the scanning algorithm.

\begin{tabular}{|c|c|c|c|c|}
\hline \multirow[t]{2}{*}{ Scanned location } & \multicolumn{2}{|c|}{ Base fluid domain (pixel/fringe) } & \multicolumn{2}{|c|}{ Test fluid domain (pixel/fringe) } \\
\hline & $\begin{array}{c}\text { Raw data } \\
\text { (pixel/fringe) } \\
10^{-3}\end{array}$ & $\begin{array}{c}\text { Inverse } \\
\text { (fringe/pixel) }\end{array}$ & $\begin{array}{c}\text { Raw data } \\
\text { (pixel/fringe) } \\
10^{-3}\end{array}$ & $\begin{array}{c}\text { Inverse } \\
\text { (fringe/pixel) }\end{array}$ \\
\hline 1 & 175 & 5.72 & 159 & 6.30 \\
\hline 2 & 175 & 5.71 & 160 & 6.24 \\
\hline 3 & 173 & 5.77 & 174 & 5.75 \\
\hline 4 & 174 & 5.74 & 158 & 6.33 \\
\hline 5 & 176 & 5.67 & 159 & 6.28 \\
\hline 6 & 165 & 6.05 & 160 & 6.27 \\
\hline 7 & 180 & 5.56 & 160 & 6.24 \\
\hline 8 & 177 & 5.66 & 162 & 6.17 \\
\hline 9 & 181 & 5.52 & 165 & 6.07 \\
\hline 10 & 174 & 5.74 & 162 & 6.17 \\
\hline
\end{tabular}


Table G.7: Measured fringe gradient data with water to water experiment.

\begin{tabular}{|c|c|c|c|c|}
\hline \multirow[t]{2}{*}{ Scanned location } & \multicolumn{2}{|c|}{ Base fluid domain (fringe/m) } & \multicolumn{2}{|c|}{ Test fluid domain (fringe/m) } \\
\hline & Heated & Deflection & Heated & Deflection \\
\hline 1 & 710 & \multirow{10}{*}{+6} & 782 & \multirow{10}{*}{-6} \\
\hline 2 & 709 & & 775 & \\
\hline 3 & 716 & & 714 & \\
\hline 4 & 713 & & 786 & \\
\hline 5 & 703 & & 779 & \\
\hline 6 & 751 & & 778 & \\
\hline 7 & 690 & & 775 & \\
\hline 8 & 703 & & 766 & \\
\hline 9 & 685 & & 753 & \\
\hline 10 & 713 & & 766 & \\
\hline
\end{tabular}


Table G.8: Fringe gradient of the measured data for both fluid domains using subtraction technique.

\begin{tabular}{|c|c|c|c|c|}
\hline Scanned location & $\begin{array}{l}\text { Base fluid } \\
\text { domain } \\
\text { (fringe/m) }\end{array}$ & $\begin{array}{c}\text { Base fluid } \\
\text { domain -Average } \\
\text { fringe gradient } \\
\text { (fringe } / \mathrm{m})\end{array}$ & $\begin{array}{l}\text { Test fluid domain } \\
\text { (fringe } / \mathrm{m} \text { ) }\end{array}$ & $\begin{array}{l}\text { Test fluid } \\
\text { domain - } \\
\text { Average } \\
\text { fringe } \\
\text { gradient } \\
\text { (fringe/m) }\end{array}$ \\
\hline 1 & 716 & \multirow{10}{*}{715} & 776 & \multirow{10}{*}{761} \\
\hline 2 & 715 & & 769 & \\
\hline 3 & 722 & & 708 & \\
\hline 4 & 719 & & 780 & \\
\hline 5 & 709 & & 773 & \\
\hline 6 & 757 & & 772 & \\
\hline 7 & 696 & & 769 & \\
\hline 8 & 709 & & 760 & \\
\hline 9 & 691 & & 747 & \\
\hline 10 & 719 & & 760 & \\
\hline
\end{tabular}




\section{G.3 $\mathrm{SiO}_{2}$-water nanofluid at $7.1 \%$ by volume concentration}

Table G.9: Data gathered with experimented performed with $\mathrm{SiO}_{2}$-water nanofluid at $7.1 \%$ by volume concentration in finite fringe mode.

\begin{tabular}{|c|c|c|c|c|c|c|c|}
\hline \multirow[t]{2}{*}{$\begin{array}{l}\text { Scanned } \\
\text { Location }\end{array}$} & \multicolumn{3}{|c|}{ Top Domain fringe gradient (bf) } & \multicolumn{3}{|c|}{$\begin{array}{c}\text { Bottom domain fringe gradient } \\
(\mathrm{tf}) \\
(\text { fringe } / \mathrm{m})\end{array}$} & \multirow{2}{*}{$\begin{array}{l}\text { Relative } \\
\text { thermal } \\
\text { conductivity }\end{array}$} \\
\hline & Unheated & Heated & $\begin{array}{l}\text { Subtraction } \\
\text { technique }\end{array}$ & Unheated & Heated & $\begin{array}{l}\text { Subtraction } \\
\text { technique }\end{array}$ & \\
\hline 1 & 875 & 1673 & 798 & 1562 & 2190 & 629 & \multirow{10}{*}{1.46} \\
\hline 2 & 906 & 1697 & 791 & 1612 & 2182 & 570 & \\
\hline 3 & 877 & 1698 & 821 & 1526 & 2168 & 642 & \\
\hline 4 & 916 & 1671 & 755 & 1587 & 2282 & 695 & \\
\hline 5 & 898 & 1701 & 803 & 1525 & 2237 & 712 & \\
\hline 6 & 895 & 1681 & 785 & 1515 & 2248 & 733 & \\
\hline 7 & 880 & 1697 & 817 & 1603 & 2298 & 696 & \\
\hline 8 & 864 & 1695 & 831 & 1552 & 2262 & 710 & \\
\hline 9 & 861 & 1693 & 832 & 1580 & 2227 & 647 & \\
\hline 10 & 865 & 1665 & 800 & 1565 & 2240 & 675 & \\
\hline
\end{tabular}




\section{G.4 $\mathrm{SiO}_{2}$-water nanofluid at $10 \%$ by volume concentration}

Table G.10: Data gathered with experimented performed with $\mathrm{SiO}_{2}$-water nanofluid at $10 \%$ by volume concentration in finite fringe mode.

\begin{tabular}{|c|c|c|c|c|c|c|c|}
\hline \multirow[t]{2}{*}{$\begin{array}{l}\text { Scanned } \\
\text { Location }\end{array}$} & \multicolumn{3}{|c|}{$\begin{array}{l}\text { Top Domain fringe gradient (bf) } \\
\text { (fringe/m) }\end{array}$} & \multicolumn{3}{|c|}{$\begin{array}{l}\text { Bottom domain fringe gradient } \\
\qquad(\mathrm{tf}) \\
(\text { fringe } / \mathrm{m})\end{array}$} & \multirow{2}{*}{$\begin{array}{c}\text { Relative } \\
\text { thermal } \\
\text { conductivity }\end{array}$} \\
\hline & Unheated & Heated & $\begin{array}{l}\text { Subtraction } \\
\text { technique }\end{array}$ & Unheated & Heated & $\begin{array}{l}\text { Subtraction } \\
\text { technique }\end{array}$ & \\
\hline 1 & 207 & 887 & 680 & 830 & 1579 & 749 & \multirow{10}{*}{1.86} \\
\hline 2 & 207 & 917 & 710 & 832 & 1566 & 734 & \\
\hline 3 & 207 & 923 & 717 & 868 & 1630 & 763 & \\
\hline 4 & 207 & 923 & 717 & 832 & 1559 & 727 & \\
\hline 5 & 207 & 917 & 710 & 839 & 1496 & 657 & \\
\hline 6 & 207 & 909 & 702 & 894 & 1528 & 635 & \\
\hline 7 & 207 & 911 & 704 & 867 & 1550 & 683 & \\
\hline 8 & 207 & 921 & 714 & 912 & 1562 & 650 & \\
\hline 9 & 207 & 904 & 697 & 825 & 1584 & 759 & \\
\hline 10 & 207 & 924 & 718 & 876 & 1561 & 684 & \\
\hline
\end{tabular}




\section{Appendix H: Esco Quote for thicker optical windows}

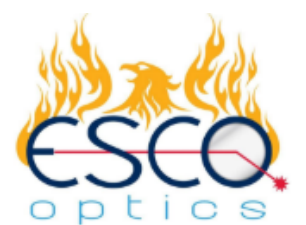

Quote Number: 36750

$\begin{aligned} & \text { Line } \text { Part/Description } \\ & 1 \text { ZP9500300 } \\ & \text { Polished Window } \\ & \text { S-BSL7 (N-BK-7 Equivalent) } \\ & \text { Diameter: } 50.0+/-0.10 \mathrm{~mm} \\ & \text { Thk: } 30.0+/-0.10 \mathrm{~mm} \\ & \text { Flatness: } 1 / 10 \text { wave } \\ & \text { S/D: } 60 / 40 \\ & \text { CA: } 90 \% \\ & \text { Bevel: } 0.50 \mathrm{~mm} \times 45 \text { degree FW typical } \\ & \\ & \text { Ship } 7 \text { weeks }\end{aligned}$

Esco Products Inc. dba Esco Optics 95 Chamberlain Road, Oak Ridge, NJ 07438

Phone: 800-922-ESCO [3726], 973-697-3700

Fax: 973-697-3011

Quotation

Page: $\quad 1$ of 1

$\begin{array}{ccr}\text { Quantity } & \underline{\text { Unit Price }} & \underline{\text { Total }} \\ 2.00 \mathrm{EA} & \$ 1,250.00 / \mathrm{EA} & \$ 2,500.00\end{array}$




\section{References}

Azmi, W. H., Sharma, K. V., Sarma, P. K., Mamat, R., Anuar, S., and Rao, V. D. (2013). Experimental determination of turbulent forced convection heat transfer and friction factor with SiO2 nanofluid. Experimental Thermal and Fluid Science, 51, 103-111.

Beer, F. P. (2009). Mechanics of materials. New York: McGraw-Hill Higher Education.

Bobbo, S., Colla, L., Scattolini, M., Agresti, F., Barison, S., Pagura, C., and Fedele, L. (2011, June). Thermal conductivity and viscosity measurements of water-based silica nanofluids. In Proceedings of the Nanotech Conference and Expo.

Buongiorno, J., Venerus, D. C., Prabhat, N., McKrell, T., Townsend, J., Christianson, R., and Bang, I. C. (2009). A benchmark study on the thermal conductivity of nanofluids. Journal of Applied Physics, 106(9), 094312.

Cengel, Y. (2014). Heat and mass transfer: fundamentals and applications. McGraw-Hill Higher Education.

Chandrasekar, M., Suresh, S., and Bose, A. C. (2010). Experimental studies on heat transfer and friction factor characteristics of $\mathrm{Al}_{2} \mathrm{O}_{3}$ /water nanofluid in a circular pipe under laminar flow with wire coil inserts. Experimental Thermal and Fluid Science, 34(2), 122-130.

Choi, S. U., and Eastman, J. A. (1995). Enhancing thermal conductivity of fluids with nanoparticles. Argonne National Lab., IL (United States).

Choi, T. Y., Maneshian, M. H., Kang, B., Chang, W. S., Han, C. S., \& Poulikakos, D. (2009). Measurement of the thermal conductivity of a water-based single-wall carbon nanotube colloidal suspension with a modified 3- $\omega$ method. Nanotechnology, 20(31), 315706.

Das, S. K., Putra, N., Thiesen, P., and Roetzel, W. (2003). Temperature dependence of thermal conductivity enhancement for nanofluids. Journal of Heat Transfer, 125(4), 567-574. 
Eastman, J. A., Choi, S. U. S., Li, S., Yu, W., and Thompson, L. J. (2001). Anomalously increased effective thermal conductivities of ethylene glycol-based nanofluids containing copper nanoparticles. Applied Physics Letters, 78(6), 718-720.

Guo, W., Li, G., Zheng, Y., and Dong, C. (2018). Measurement of the thermal conductivity of $\mathrm{SiO}_{2}$ nanofluids with an optimized transient hot wire method. Thermochimica Acta, 661, 84-97.

Hamilton, R. L., and Crosser, O. K. (1962). Thermal conductivity of heterogeneous twocomponent systems. Industrial \& Engineering Chemistry Fundamentals, 1(3), 187-191.

Haridas, D., Rajput, N. S., and Srivastava, A. (2015). Interferometric study of heat transfer characteristics of $\mathrm{A} 12 \mathrm{O} 3$ and $\mathrm{SiO} 2$-based dilute nanofluids under simultaneously developing flow regime in compact channels. International Journal of Heat and Mass Transfer, 88, 713-727.

Huang, X. F., and Li, S. J. (2014). Near-Field Nanofluid Concentration Measurement by Rayleigh Particle Scattering Bragg Grating Evanescent Wave. International Journal of Optomechatronics, 8(2), 100-113.

Hwang, K. S., Jang, S. P., and Choi, S. U. (2009). Flow and convective heat transfer characteristics of water-based $\mathrm{Al} 2 \mathrm{O} 3$ nanofluids in fully developed laminar flow regime. International Journal of Heat and Mass Transfer, 52(1-2), 193-199.

J. C. Maxwell, A Treatise on Electricity and Magnetism. UK: Clarendon. (1973)

Kakaç, S., and Pramuanjaroenkij, A. (2009). Review of convective heat transfer enhancement with nanofluids. International Journal of Heat and Mass Transfer, 52(13-14), 3187-3196.

Kang, H. U., Kim, S. H., and Oh, J. M. (2006). Estimation of thermal conductivity of nanofluid using experimental effective particle volume. Experimental Heat Transfer, 19(3), 181-191.

Khalili, E., Saboonchi, A., and Saghafian, M. (2017). Experimental study of nanoparticles distribution in natural convection of $\mathrm{Al}_{2} \mathrm{O}_{3}$-water nanofluid in a square cavity. International Journal of Thermal Sciences, 112, 82-91.

Kleinstreuer, C., and Feng, Y. (2011). Experimental and theoretical studies of nanofluid thermal conductivity enhancement: a review. Nanoscale Research Letters, 6(1), 229. 
Kline, S.J. and McClintock, F.A. (1953), "Describing Experimental Uncertainties in SingleSample Experiments," Mechanical Engineering, Vol. 75, pp. 3-8.

Murshed, S. M. S., Leong, K. C., and Yang, C. (2005). Enhanced thermal conductivity of TiO2 — water based nanofluids. International Journal of Thermal Sciences, 44(4), 367-373.

Naylor, D., Floryan, J.M., and Tarasuk, J.D. (1991). A Numerical study of Developing Free

Convection Between Isothermal Vertical Plates, Journal of Heat Transfer, Vol. 113, pp.

$620-626$.

Nguyen, C. T., Roy, G., Gauthier, C., and Galanis, N. (2007). Heat transfer enhancement using Al2O3-water nanofluid for an electronic liquid cooling system. Applied Thermal Engineering, 27(8-9), 1501-1506.

Parker Hannifin Corp. (2019). O-Ring Compression Force. Retrieved from https://promo.parker.com/promotionsite/oring-ehandbook/us/ehome/ci.O\%E2\%80\%93RingCompression-Force,EN.EN

Peñas, J. R. V., Ortiz de Zarate, J. M., and Khayet, M. (2008). Measurement of the thermal conductivity of nanofluids by the multicurrent hot-wire method. Journal of Applied Physics, 104(4), 044314.

Rajput, N. S., and Srivastava, A. (2016). Non-intrusive dynamic measurements of nanofluidbased heat transfer phenomena under thermally developing flow regime in the context of compact channels. Experimental Thermal and Fluid Science, 74, 271-285.

Rao, S. S., and Srivastava, A. (2014). Interferometry-based whole field investigation of heat transfer characteristics of dilute nanofluids. International Journal of Heat and Mass Transfer, 79, 166-175.

Roeleveld, D. (2013). Experimental and Numerical Study of Free Convection in a Vertical Channel With Opposing Buoyancy Forces (Doctoral dissertation, Ph. D. thesis, Ryerson University, Toronto). 
Rusconi, R., Rodari, E., and Piazza, R. (2006). Optical measurements of the thermal properties of nanofluids. Applied Physics Letters, 89(26), 261916.

Ryzhkov, I. I., and Minakov, A. V. (2014). The effect of nanoparticle diffusion and thermophoresis on convective heat transfer of nanofluid in a circular tube. International Journal of Heat and Mass Transfer, 77, 956-969.

Sharma, K. V., Sarm, P. K., Azmi, W. H., Mamat, R., and Kadirgama, K. (2012). Correlations to predict friction and forced convection heat transfer coefficients of water based nanofluids for turbulent flow in a tube. International Journal of Microscale and Nanoscale Thermal and Fluid Transport Phenomena, 3(4), 283.

Shukla, R. K., and Dhir, V. K. (2008). Effect of Brownian motion on thermal conductivity of nanofluids. Journal of Heat Transfer, 130(4), 042406.

Silicon Glass $\left(\mathrm{SiO}_{2}\right)$. (2012). Retrieved from https://www.crystran.co.uk/opticalmaterials/silica-glass-sio2

Slepicka, J. S., and Cha, S. S. (1995). Stabilized nonlinear regression for interferogram analysis. Applied optics, 34(23), 5039-5044.

Taha-Tijerina, J. J. (2018). Thermal Transport and Challenges on Nanofluids Performance. In Microfluidics and Nanofluidics. IntechOpen.

Tarasuk, J.D. (1968). "The Theory, Design and Operation of the University of Saskatchewan 8Inch Mirror Mach-Zehnder Interferometer," Technical Report C-3, Mechanical Engineering Department, University of Saskatchewan.

Tavman, I., and Turgut, A. (2010). An investigation on thermal conductivity and viscosity of water based nanofluids. In Microfluidics Based Microsystems (pp. 139-162). Springer, Dordrecht.

Touloukian, Y.S, Liley, P.E, and Saxena, S.C. (1970). Thermophysical Properties of MatterThe TRPC Data Series. Volume 3. Thermal Conductivity - Nonmetallic Liquids and Gases. 
Touloukian, Y.S. and Makita, T. (1975). "Specific Heat: Nonmetallic Liquids and Gases," Thermophysical Properties of Matter, Vol. 6, Thermophysical Properties Research Center (TPRC), Purdue University, Plenum Publishing Corp.

Touloukian, Y.S., Saxena, S.C., and Hestemians, P. (1975). "Viscosity: Nonmetallic Liquids and Gases," Thermophysical Properties of Matter, Vol. 11, Thermophysical Properties Research Center (TPRC), Purdue University, Plenimi Publishing Corp.

Tsai, T. H., Kuo, L. S., Chen, P. H., and Yang, C. T. (2008). Effect of viscosity of base fluid on thermal conductivity of nanofluids. Applied Physics Letters, 93(23), 233121.

Von Bistram, P. (1995) "The Design, Development and Preliminary Testing of the Ryerson Mach-Zehnder Interferometer,” B.Eng. Thesis, Ryerson Polytechnic University.

Wang, X., Xu, X., and S. Choi, S. U. (1999). Thermal conductivity of nanoparticle-fluid mixture. Journal of Thermophysics and Heat Transfer, 13(4), 474-480.

Wen, D., and Ding, Y. (2004). Experimental investigation into convective heat transfer of nanofluids at the entrance region under laminar flow conditions. International journal of heat and Mass Transfer, 47(24), 5181-5188.

White, Frank M. Fluid Mechanics. Boston, Mass: WCB/McGraw-Hill (1999). Print.

Xie, H., Yu, W., Li, Y., and Chen, L. (2011). Discussion on the thermal conductivity enhancement of nanofluids. Nanoscale Research Letters, 6(1), 124.

Xuan, Y., and Li, Q. (2003). Investigation on convective heat transfer and flow features of nanofluids. Journal of Heat transfer, 125(1), 151-155.

Yousefi, T., Farahbakhsh, B., Naylor, D., and Saghir, M. Z. (2015). Thermal field visualization of nanofluids using full-field interferometry. International Communications in Heat and Mass Transfer, 65, 125-129.

Yousefi,T., and Naylor, D. (2019). An experimental study on the refractive index and temperature coefficient of refractive index for $\mathrm{Al}_{2} \mathrm{O}_{3}$ and $\mathrm{SiO}_{2}$ nanofluids. Paper in preparation. 
Yu, W., and Choi, S. U. S. (2003). The role of interfacial layers in the enhanced thermal conductivity of nanofluids: a renovated Maxwell model. Journal of nanoparticle research, 5(12), 167-171.

Yunus, C. A., and Afshin, J. G. (2011). Heat and mass transfer: fundamentals and applications. Tata McGraw-Hill, New Delhi, India.

Zeng, Y. X., Zhong, X. W., Liu, Z. Q., Chen, S., and Li, N. (2013). Preparation and enhancement of thermal conductivity of heat transfer oil-based $\mathrm{MoS}_{2}$ nanofluids. Journal of Nanomaterials, 2013, 3. 